Check for updates

Cite this: RSC Adv., 2018, 8, 31803

\title{
Sulfur(vi) fluoride exchange as a key reaction for synthesizing biaryl sulfate core derivatives as potent hepatitis $C$ virus NS5A inhibitors and their structure-activity relationship studies $\uparrow$
}

\author{
Youngsu You, ${ }^{a}$ Hee Sun Kim, ${ }^{b}$ Jung Woo Park, ${ }^{c}$ Gyochang Keum, ${ }^{d}$ Sung Key Jang ${ }^{e}$ \\ and B. Moon Kim (D) *a
}

\begin{abstract}
Extremely potent, new hepatitis C virus (HCV) nonstructural 5A (NS5A) featuring substituted biaryl sulfate core structures was designed and synthesized. Based on the previously reported novel HCV NS5A inhibitors featuring biaryl sulfate core structures which exhibit two-digit picomolar half-maximal effective concentration ( $\mathrm{EC}_{50}$ ) values against HCV genotype $1 \mathrm{~b}$ and $2 \mathrm{a}$, the new inhibitors equipped with the sulfate core structures containing diversely substituted aryl groups were explored. In this study, highly efficient, chemoselective coupling reactions between an arylsulfonyl fluoride and an aryl silyl ether, known as the sulfur(vi) fluoride exchange (SuFEx) reaction, were utilized. Among the inhibitors prepared based on the SuFEx chemistry, compounds 14, 15 and 29 exhibited two-digit picomolar EC 50 values against GT-1b and single digit or sub nanomolar activities against the HCV GT-2a strain. Nonsymmetrical inhibitors containing an imidazole and amide moieties on each side of the sulfate core structures were also synthesized. In addition, a biotinylated probe targeting NS5A protein was prepared for labeling using the same synthetic methodology.
\end{abstract}

Received 26th June 2018
Accepted 28th August 2018

DOI: $10.1039 / c 8 r a 05471 a$

rsc.li/rsc-advances

Currently, six NS5A inhibitors are on the market for use with

\section{Introduction}

Hepatitis C virus (HCV) is known to cause serious liver infection, which may result in liver cirrhosis and eventually hepatocellular carcinoma (HCC).$^{1-7}$ Currently, more than 170 million people are infected with HCV worldwide, and approximately 3 to 4 million people are newly infected by this insidious disease yearly. ${ }^{8-16}$

In recent years, many pharmaceutical companies and laboratories worldwide have focused their studies on understanding the HCV life cycle and developing direct-acting agents (DAAs) as next-generation therapies. ${ }^{17-22}$ One of the most notable $\mathrm{HCV}$ target proteins is the nonstructural 5A (NS5A) protein, which is believed to be involved in viral replication and assembly of new virions. $^{23-29}$

\footnotetext{
${ }^{a}$ Department of Chemistry, College of Natural Sciences, Seoul National University, Seoul 08826, South Korea. E-mail: kimbm@snu.ac.kr

${ }^{b}$ Division of Integrative Biosciences and Biotechnology, Pohang University of Science and Technology, Pohang 37673, South Korea

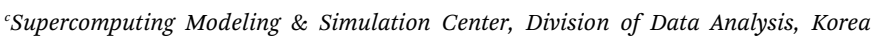
Institute of Science and Technology Information (KISTI), 245 Daehak-ro, Yuseong-gu, Daejeon, 34141, South Korea

${ }^{d}$ Center for Neuro-Medicine, Brain Science Institute, Korea Institute of Science and Technology (KIST), Hwarangno 14-gil 5, Seongbuk-gu, Seoul 02455, South Korea ${ }^{e}$ Department of Life Sciences, Pohang University of Science and Technology, Pohang 37673, South Korea
}

$\dagger$ Electronic supplementary information (ESI) available. See DOI: 10.1039/c8ra05471a ibavirin, NS3/NS4A protease inhibitors, or NS5B inhibitors. ${ }^{7,30-32}$ In 2014, the US Food and Drug Administration (FDA) approved ledipasvir (GS-5885) with the NS5B inhibitor, sofosbuvir. ${ }^{33,34}$ Ombitasvir (ABT-267) was also approved by the FDA in combination with paritaprevir (NS3/4A protease inhibitor), ritonavir (protease inhibitor), and dasabuvir (NS5B inhibitor) for HCV genotype 1 infections. ${ }^{35-37}$ Daclatasvir (BMS-790052) in combination with sofosbuvir (2015), elbasvir (MK-8742) in combination with grazoprevir (NS3/4A protease inhibitor), and velpatasvir (GS-5816) along with sofosbuvir in 2016 are among other FDA-approved HCV DAAs. ${ }^{38-41}$ Recently, the FDA approved Vosevi ${ }^{\circledR}$ in 2017, which is a combination of sofosbuvir, velpatasvir, and voxilaprevir (NS3/4A inhibitor); a new pan-genotype drug, Mavyret ${ }^{\circledR}$ (glecaprevir/pibrentasvir), was also approved in the same year. ${ }^{32,42,43}$

Daclatasvir, which was first reported in 2010, exhibits extremely high antiviral activities against a few genetic variants, and against genotype $1 \mathrm{~b}$ with $\mathrm{EC}_{50}$ value of $9 \mathrm{pM}^{44-48}$ Therefore, numerous DAAs have been introduced based on the daclatasvir structure, which has a symmetrical phenylimidazole core with proline-valine-carbamate motif. ${ }^{49-53}$ Novel, excessively potent NS5A inhibitors have been introduced by many pharmaceutical companies. ${ }^{54-58}$ However, treatment with daclatasvir quickly resulted in the development of drug-resistance, such as mutation on L31 and Y93 residues in NS5A protein and the antiviral activities were reduced by up to 15000 times. $^{59-63}$ 
We recently reported the design, synthesis, and structureactivity-relationship (SAR) studies of novel NS5A inhibitors based on a biaryl sulfate core structure (Fig. 1). ${ }^{64}$ These compounds showed high antiviral activities and good additive effects as a combination treatment with an NS5B inhibitor, sofosbuvir, without showing any cytotoxicity. ${ }^{64}$

Encouraged by the initial results, we optimized the biaryl sulfate core part and carried further SAR studies focused on the sulfate core structures. During this investigation, we discovered that some substituted biaryl sulfate core structures were not readily accessible using traditional coupling reactions with sulfonyl diimidazole (SDI). For example, in the cross-coupling reactions of $o$-fluorophenol derivatives with SDI, only a monomeric aryl sulfonyl imidazole was obtained. Likewise, the sulfonyl diimidazole coupling strategy would not be appropriate for selective construction of nonsymmetrical biaryl sulfate core structures (Fig. 2).

Although the first-generation DAA HCV drugs have symmetrical structures similar to daclatasvir, many of the nextgeneration DAAs developed to treat multi-genotype strains tend to possess nonsymmetric structures. ${ }^{65-69}$ Since there have been reports of NS5A inhibitors with a high degree of variation at the center core, we envisioned that nonsymmetrical biaryl sulfate core structures with substituted aryl groups would be worth investigating and, thus, were included in our SAR studies. ${ }^{69-73}$ For the synthesis of the nonsymmetrical biaryl sulfate core structures, SuFEx chemistry appeared to be an extremely attractive strategy. ${ }^{74}$ Here, we report the fruitful exploitation of

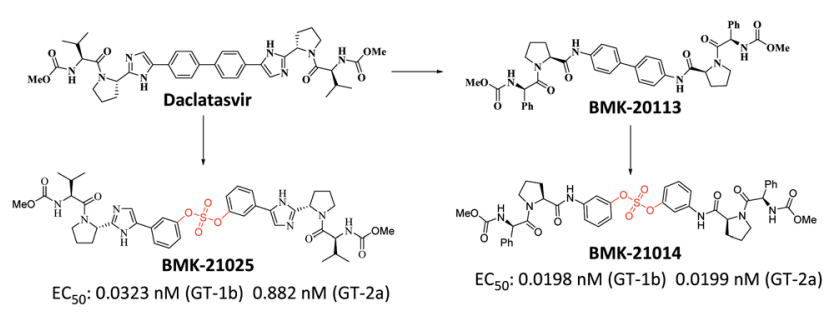

Fig. 1 Discovery of biaryl sulfate derivatives as potent HCV NS5A inhibitors.

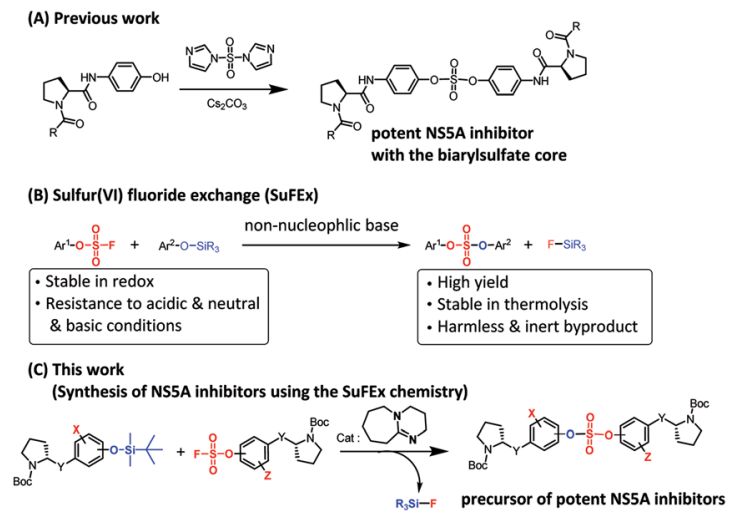

Fig. 2 Biaryl sulfate core based nonstructural 5A (NS5A) inhibitors and modification using sulfur(vi) fluoride exchange (SuFEx) chemistry.
SuFEx chemistry for the construction of symmetrical and nonsymmetrical biaryl sulfate-based HCV NS5A inhibitors, which exhibit extremely high inhibitory activities.

In 2014, Sharpless and coworkers introduced the SuFEx click reaction for chemoselectively linking two different phenol derivatives. ${ }^{74,75}$ The sulfate coupling reaction was accomplished through the reaction of an aryl fluorosulfate and an aryl silyl ether in the presence of a catalytic amount of 1,8diazabicyclo(5.4.0)undec-7-ene (DBU). ${ }^{76-78}$ The synthesis of sulfate compounds from the $\mathrm{S}^{\mathrm{VI}}-\mathrm{F}$ group proceeded with high yields and at a fast rate because of the driving force that formed the strong $\mathrm{Si}-\mathrm{F}$ and $\mathrm{S}-\mathrm{O}$ bond. ${ }^{74}$ Furthermore, the resulting compounds exhibited considerably high stabilities against hydrolysis, nucleophilic substitution, thermolysis, and reduction..$^{74,77-79}$ Moreover, this useful coupling reaction produces only inert silyl fluorides as by-products. ${ }^{75,78,80,81}$ We envisioned that this chemoselective SuFEx reaction could be used for the synthesis of novel HCV NS5A inhibitors containing biaryl sulfates possessing various substitution at the $o$-position of the phenol derivatives or a nonsymmetric biaryl sulfate core structures.

\section{Results and discussion}

We prepared various aminophenol derivatives (3a-d) as substrates for the SuFEx reaction (Scheme 1). The reduction of nitro groups to amines was carried out with commercially available nitrophenols 1a-b using $10 \mathrm{wt} \%$ palladium on activated charcoal $(\mathrm{Pd} / \mathrm{C}) .{ }^{82}$ The amide linked monomers $3 \mathbf{a}-\mathbf{d}$ were prepared from amino-phenol derivatives $2 \mathrm{a}-\mathbf{d}$ and $\mathrm{N}$-Boc-Lproline using general amide coupling methods in the presence of 1-ethyl-3-(3-dimethylaminopropyl)carbodiimide (EDCI). ${ }^{83,84}$ The imidazole-linked monomers $7 \mathbf{a}-\mathbf{c}$ were prepared from the acetophenone derivatives following an established procedure. $^{64,85} \alpha$-Bromination of acetophenone 4 was carried out in the presence of copper(II) bromide to provide $\alpha$-bromoacetophenone 5 in an $81 \%$ yield. ${ }^{85,86}$ Then, the resulting product 5 was treated with $N$-Boc-L-proline in the presence of DIPEA to yield ester $6 \mathbf{a}^{85}$ Likewise, commercially available $\alpha$-bromoacetophenone derivatives $\mathbf{5 b}$ and $\mathbf{5 c}$ were converted to the corresponding esters $\mathbf{6 b}$ and $\mathbf{6 c}$, respectively. Synthesis of the imidazole derivatives $\mathbf{7 a - c}$ was accomplished by the reaction of 6a-c with ammonium acetate. ${ }^{85}$

The introduction of a fluorosulfonyl (fosyl) group was achieved by the reaction of the corresponding phenol derivatives with sulfuryl fluoride $\left(\mathrm{SO}_{2} \mathrm{~F}_{2}\right)$ and 1.7 equiv. DIPEA in dichloromethane (Table 1). ${ }^{74}$ Since $\mathrm{SO}_{2} \mathrm{~F}_{2}$ is a gas, the reaction mixture was stirred rapidly (>1000 rpm) to enhance the liquidgas contact. ${ }^{76,78}$ The reaction was complete within $5 \mathrm{~h}$, monitored using thin layer chromatography (TLC). The structures of the fosylated products were confirmed using proton $\left({ }^{1} \mathrm{H}\right),{ }^{13} \mathrm{C}$, and ${ }^{19} \mathrm{~F}$ nuclear magnetic resonance (NMR) spectroscopy; especially, ${ }^{19} \mathrm{~F}$ NMR chemical shifts of fluorine at fosyl group ranged from $c a$. 31 to $40 \mathrm{ppm} .{ }^{76,87}$ These fluorosulfate derivatives exhibited good stability against hydrolysis and could be stored on the shelf for months. ${ }^{77,78,80,88}$ Compounds 8a-g were obtained in good yields (>80\%) as shown in Table 1. 

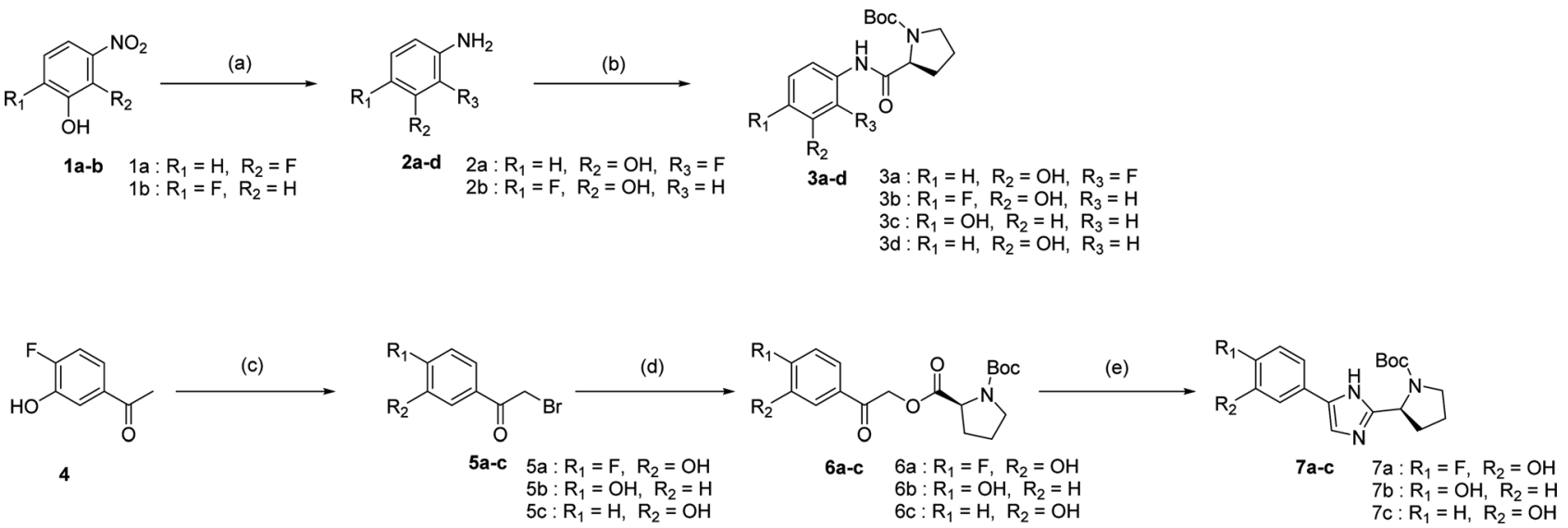

Scheme 1 Synthesis of intermediates; reagents and conditions: (a) Pd/C, $\mathrm{H}_{2}, \mathrm{MeOH}$, room temperature (rt), $24 \mathrm{~h}, 99 \%$; (b) EDCl, $\mathrm{N}$-Boc-Lproline, $\mathrm{CH}_{2} \mathrm{Cl}_{2}, \mathrm{rt}, 4 \mathrm{~h}, 59-96 \%$; (c) $\mathrm{CuBr}_{2}, \mathrm{EA} / \mathrm{CHCl}_{3}$, reflux, $8 \mathrm{~h}, 81 \%$; (d) $\mathrm{N}$-Boc-L-proline, $\mathrm{N}, \mathrm{N}$-diisopropylethylamine (DIPEA), acetonitrile, rt, 5 h, 72-91\%; (e) $\mathrm{NH}_{4} \mathrm{OAc}$, toluene, $95^{\circ} \mathrm{C}, 20 \mathrm{~h}, 45-52 \%$.

Table 1 Preparation of fluorosulfate monomers ${ }^{a}$
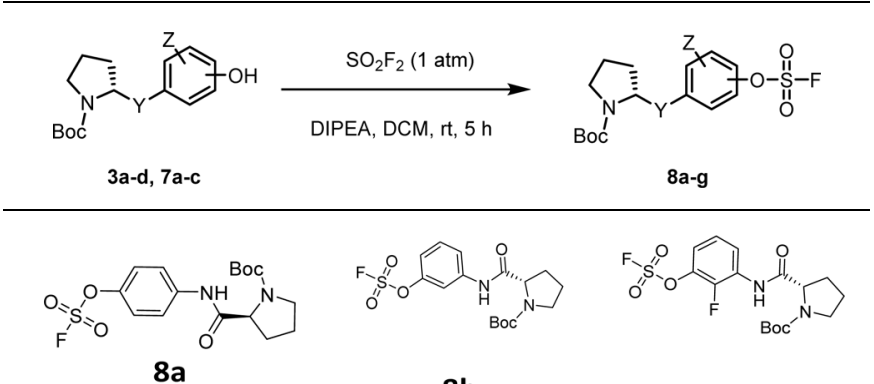

$(90 \%)$
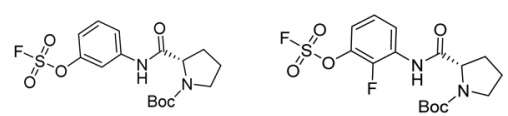

8b

$(87 \%)$

$8 \mathrm{c}$

$(83 \%)$
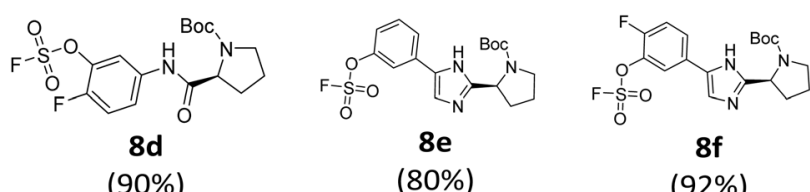

(92\%)

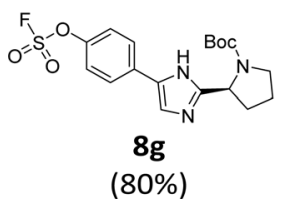

${ }^{a}$ Yields of isolated products.

We then constructed the sulfate core structures using aryl silyl ether counterparts through SuFEx chemistry (Table 2). ${ }^{79}$ Silylation of phenol groups was achieved using the standard method with tert-butyldimethylsilyl chloride (TBDMSCl) and imidazole. ${ }^{89}$ The coupling reaction between an aryl fosylate and aryl silyl ether was carried out in the presence of DBU in dimethyl formamide (DMF) according to Sharpless' protocol. ${ }^{7,75}$ Then, the mixture was stirred at $50{ }^{\circ} \mathrm{C}$ for $12 \mathrm{~h}$. The biaryl sulfate products (compounds 10a-10f) were formed in good to excellent yields in the presence of catalytic amounts of DBU (20-30 mol\%). ${ }^{74}$ However, in case of the coupling reactions involving the silyl ether or fluorosulfate

monomer containing an imidazole moiety (compounds $\mathbf{1 0 g}-$ 10j), 2.2-2.3 equiv. DBU was used for the SuFEx coupling products.

We synthesized nonsymmetric or $o$-fluoro substituted biaryl sulfate based NS5A inhibitors using appropriate precursors (Tables 3 and 4). The tert-butyloxycarbonyl (Boc)-protecting group for the proline moiety was deprotected with $50 \%(\mathrm{v} / \mathrm{v})$ TFA in $\mathrm{CH}_{2} \mathrm{Cl}_{2} \cdot{ }^{64,90,91}$ Volatiles were removed from the reaction mixture under reduced pressure and the residue was directly coupled with a capping group such as $N$-methyloxycarbonylprotected valine (val) or phenylglycine (phg) in the presence of EDCI and HOBt. ${ }^{64}$ Finally, the biaryl sulfate based HCV NS5A inhibitors were obtained after purification of the crude products using silica gel chromatography. ${ }^{64}$

To determine the antiviral activities of each compound, we measured the $\mathrm{EC}_{50}$ of inhibitors for genotype-1b (GT-1b) and $2 \mathrm{a}$ (GT-2a) using the HCV replicon and HCV cell culture (HCVcc) systems, respectively. ${ }^{91}$ We used the human hepatoma Huh 7.5.1 cell line to investigate the $\mathrm{EC}_{50}$ of the compounds in the HCV replicon systems for GT-1b, which encodes the bicistronic NK5.1 gene and Gaussia luciferase (Gluc) reporter gene. ${ }^{\mathbf{9} 91-93}$

On the other hand, the inhibitory activities of GT-2a were measured using the HCVcc system with JFH 5a-Rluc-ad34, which is a derivative of JFH1 containing a Renilla luciferase reporter and cell culture adaptive mutations..$^{\mathbf{9 0 9 4 , 9 5}}$

The antiviral activities $\left(\mathrm{EC}_{50}\right)$ of the tested compounds containing amide groups on both side of the sulfate core against replicon (GT-1b) and HCVcc (GT-2a) are listed in Table 3. We first selected the compounds that exhibited $<1 \mathrm{nM} \mathrm{EC}_{50}$ for GT$1 \mathrm{~b}$, and then measured the HCVcc $\mathrm{EC}_{50}$ for GT-2a. In the amide linker series, the D-valine or D-phenylglycine derivatives were chosen as only capping groups because these groups showed the highest levels of antiviral activities in our previous study. ${ }^{\mathbf{6 4}}$

We first carried out the SAR studies of inhibitors containing a non-symmetric sulfate core. The inhibitor $\mathbf{1 1}$ (Table 3, entry 5) containing 3-aminophenyl (4-aminophenyl) sulfate derivative equipped with a D-phenylglycine capping moiety showed 36 -fold lower potency against the GT-1b than the symmetric $p, p^{\prime}$ - 
Table 2 Preparation of biaryl sulfate core structures using sulfur(vı) fluoride exchange (SuFEx) chemistry ${ }^{a}$

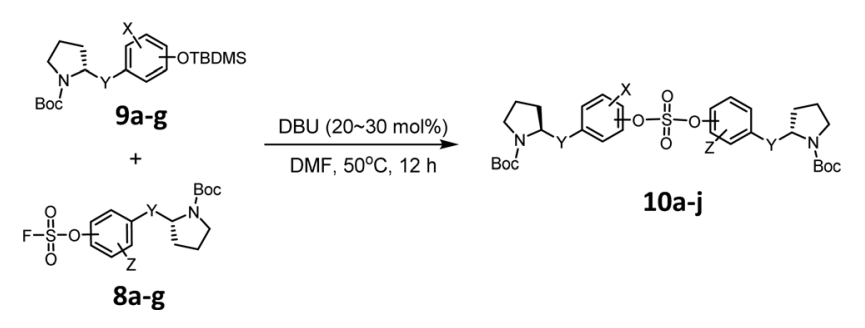

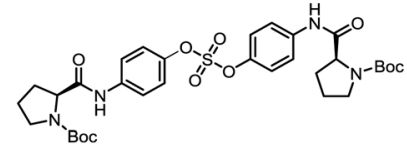

$10 a$

(90\%)

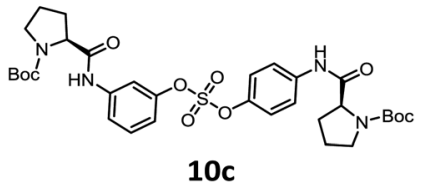

(93\%)

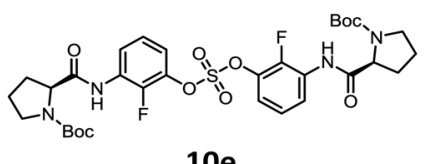

(98\%)

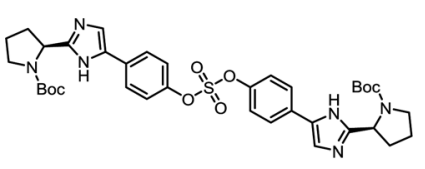

$10 \mathrm{~g}$

(69\%)

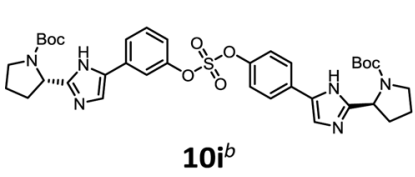

(52\%)
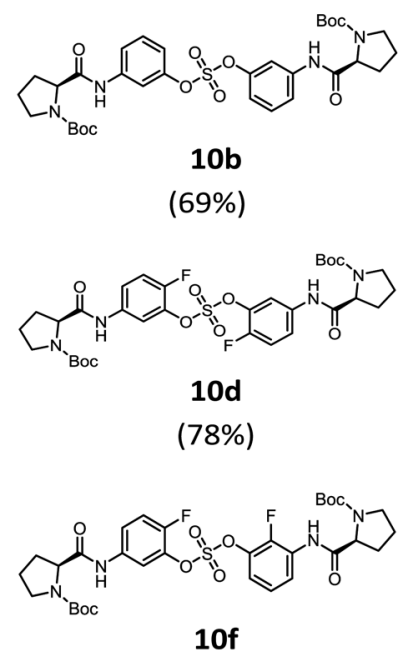

$(58 \%)$

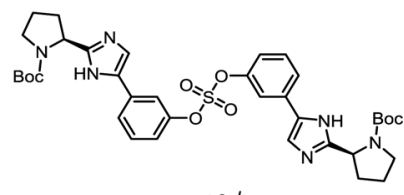

$10 h^{b}$

(67\%)

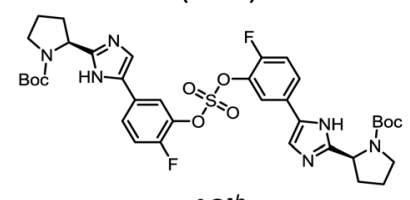

$10 \mathbf{j}^{b}$

(63\%)
${ }^{a}$ Yields of isolated products. ${ }^{b}$ The reactions were carried out with DBU (2.2-2.3 equiv.).

substituted inhibitor BMK-21007 did, while the antiviral activities on GT-2a were reduced by $>6$-fold compared to those of the symmetrically $m, m^{\prime}$-substituted biaryl sulfate inhibitor BMK21014 (Table 3, entries 1 and 2, respectively). ${ }^{64}$ The replacement of the D-phenylglycine with $\mathrm{D}$-valine moieties as shown in compound 12 induced no apparent inhibitory activity against GT-1b at $100 \mathrm{nM}$ concentration (Table 3, entry 6).

We next investigated the inhibitors containing $o$-fluoro substituted biaryl groups, expecting increased potency based on the restricted rotational conformation around the sulfate core. In a previous study, compound BMK-21028 (Table 3, entry 3), which was substituted with methyl at 2- and 2'-positions, showed activity at two-digit nanomolar levels in GT-1b. ${ }^{64}$ However, replacement of the methyl groups of BMK-21028 with fluorine (compound 13) improved the inhibitory activity against GT-1b strain with 0.0204 nM EC $_{50}$ (Table 3, entry 7). This result, surprisingly, was a 1127 -fold higher inhibitory activity than that of BMK-21028 was, suggesting that the $o$-position in the biaryl groups is extremely important for increasing the potency.

The $C_{2}$ symmetric 6,6'-difluoro-substituted phenol derivatives containing the D-phenylglycine moieties 14 (Table 3, entry 8) also showed increased inhibitory activities against both GT1b and GT-2a, compared to BMK-21020, which has $o$-methyl groups at 6- and 6'-positions of the biaryl groups (Table 3, entry 4). ${ }^{64}$ Although the inhibitory activities against GT-1b were increased, compound 14 showed low GT-2a inhibitory activity (single nanomolar level $\mathrm{EC}_{50}$ ) and compound 13 showed no inhibitory activities at $10 \mathrm{nM}$.

Nonsymmetrically substituted difluorobiarylsulfate $\mathbf{1 5}$ (Table 3, entry 9) with 2- and 2 '-fluoro-substitution exhibited 5-fold weaker activity for GT-1b and 2-fold higher potency for GT-2a compared to symmetric compound 13. The replacement of the D-phenylglycine with D-valine moiety (compounds 16 and 17) showed lower inhibitory activities except for compound 18, which had GT-1b potency to single nanomolar range (Table 3 , entries 10,11 , and 12 , respectively). The fact that the SAR results were highly dependent on the substitution and the sulfate linking the position of the biaryl groups indicates that the inhibitory activity of various HCV genotype is highly sensitive to the structural features of the core moiety. These results indicate that ortho-substitution in a phenyl group with fluorine blocks the free rotation, which lowers the antiviral activities against GT-1b. Nevertheless, replacing it with hydrogen, which lacks steric hindrance relatively, enhanced the inhibitory activities against the GT-2a gene.

As shown in Table 2, we designed the NS5A inhibitor series consisting of imidazole linkers with the aim of enhancing the antiviral activities and solubility. ${ }^{\mathbf{9 6 - 9 8}}$ In our previous report, the imidazole linked biaryl sulfate inhibitors containing an L-valine capping group (BMK-21025, Table 4, entry 1) showed good activities against GT-1b and GT-2a. ${ }^{64}$ Thus, we introduced nonsymmetric or substituted sulfate core structures in imidazole-linked inhibitors to determine if it would influence the inhibitory activities and the results are summarized in Table 4. Compound 19, containing $o$-fluoro substituted biaryl sulfate with D-valine derivative, showed no inhibitory activity at $100 \mathrm{nM}$ against replicon GT-1b (Table 4, entry 2). Compound 20 with an $\mathrm{N}$-methoxycarbonyl-L-valine capping group resulted in twodigit $\mathrm{nM} \mathrm{EC}_{50}$ on GT-1b (Table 4, entry 3). The replacement of the L-valine moiety with D-phenylglycine moiety as in compound 21 dramatically enhanced the antiviral activity against GT-1b (Table 4, entry 4). However, these results showed 25-fold lower activity than that of inhibitors linked with amides such as compound 15. Introduction of $m$-, $p^{\prime}$-disubstituted biaryl sulfate core as in compound 22 demonstrated that the potencies for both GT-1b and GT-2a were better than that of compound 21 with an $o$-fluoro substituent (Table 4 , entry 5 ). While the D-valine derivative 23 showed no inhibition at $100 \mathrm{nM}$, the opposite stereoisomeric L-valine derivative $\mathbf{2 4}$ showed improved potency 
Table 3 Synthesis and in vitro antiviral activities of inhibitors comprising an amide linked biaryl sulfate core ${ }^{a}$

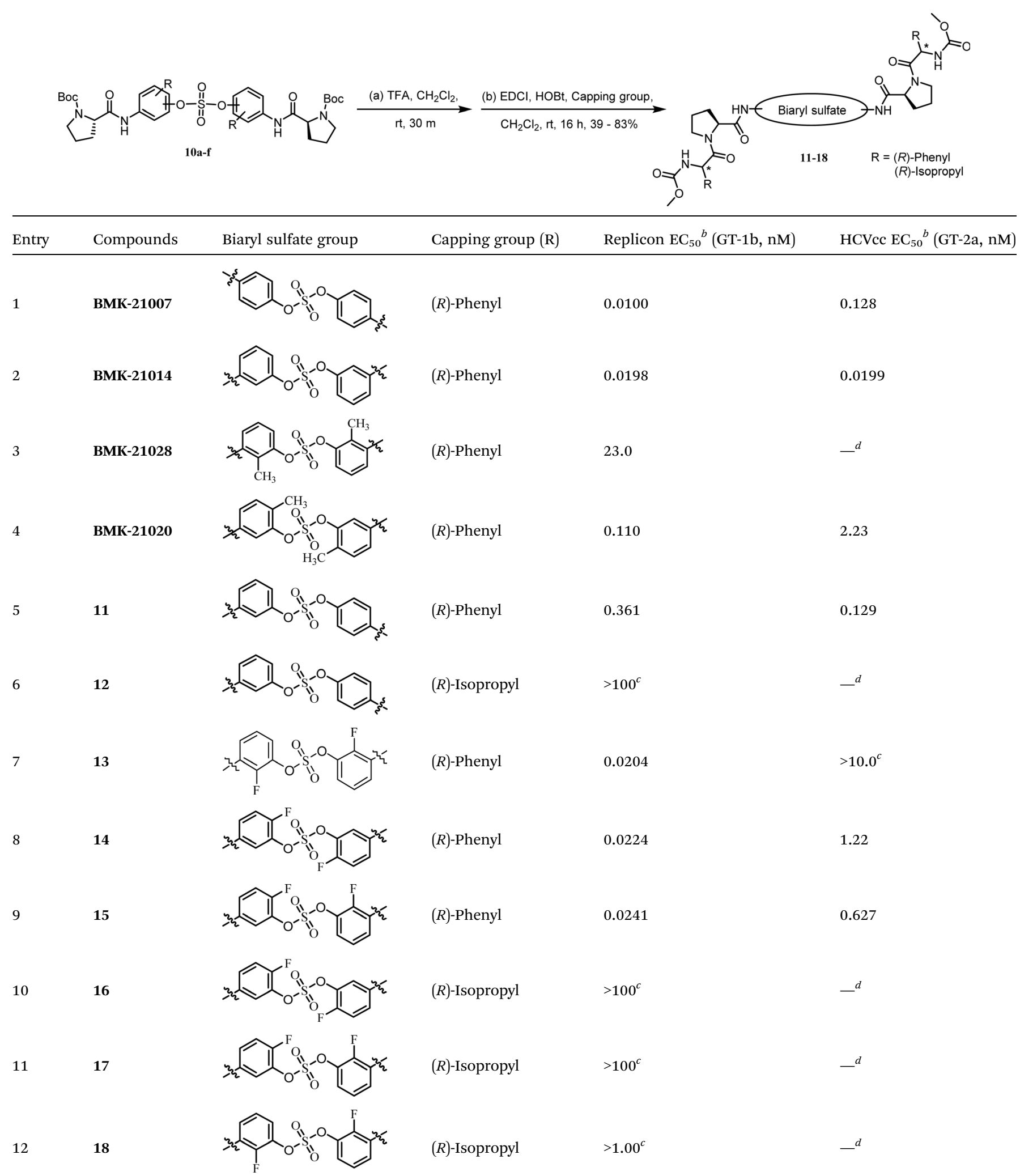

${ }^{a} \mathrm{EC}_{50}$, half-maximal (50\%) effective concentration; GT-1b, genotype-1b; GT-2a, genotype-2a. ${ }^{b}$ All experiments were performed three times except for compounds that exhibited $>1 \mathrm{nM} \mathrm{EC}_{50}$ for GT-1b. ${ }^{c}$ Experiment was performed once. ${ }^{d}$ Not determined. 


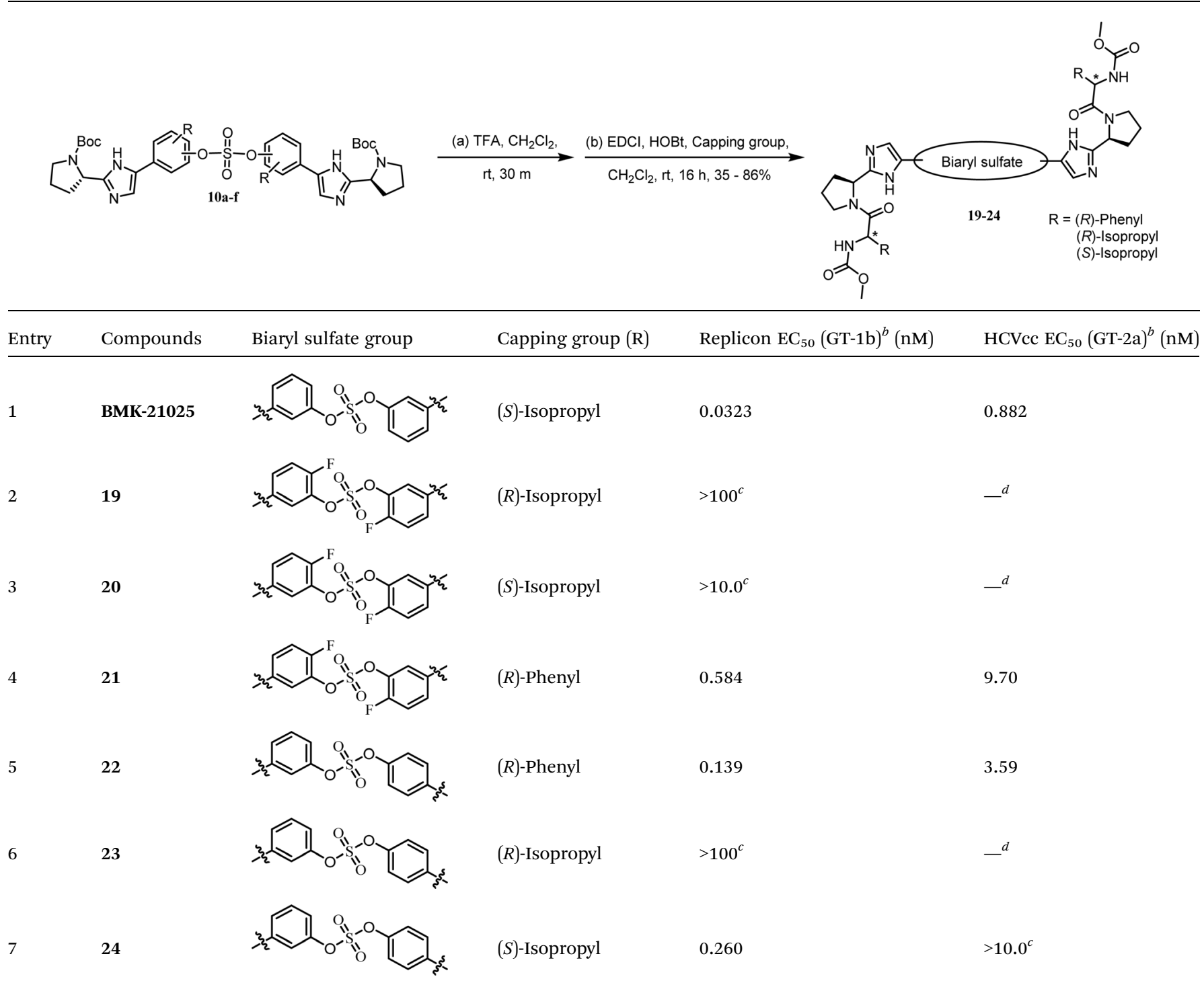

${ }^{a} \mathrm{EC}_{50}$, half-maximal (50\%) effective concentration; GT-1b, genotype-1b; GT-2a, genotype-2a. ${ }^{b}$ All experiments were performed three times except for compounds that exhibited $>1 \mathrm{nM} \mathrm{EC}_{50}$ for GT-1b. ${ }^{c}$ Experiment was performed once. ${ }^{d}$ Not determined.

for GT-1b (Table 4, entries 6 and 7, respectively). However, it showed no marked antiviral activity against GT-2a.

Based on the SAR study, it can be concluded that compounds with asymmetric biaryl sulfate core and D-phenylglycine capping derivatives (compounds $\mathbf{1 1}$ and 22) showed the best activities for both genotypes. We proceeded to synthesize nonsymmetric compounds, exploiting the chemoselectivity of SuFex chemistry. In our previous study, we reported that $m, m^{\prime}$-biaryl sulfate derivatives exhibit high inhibitory activities against NS5A. ${ }^{64}$ Especially, $C_{2}$-symmetric biaryl sulfate comprising amide (BMK21014) and imidazole (BMK-21025) linkers showed extremely high antiviral activities against two genotypes. Based on these results, we synthesized compound $\mathbf{2 9}$ having a hybridized structure of the two compounds. Due to the orthogonality of SuFEx, we were able to synthesize the biaryl sulfate compound containing imidazole-proline-L-valine carbamate derivative and amide-
proline-D-phenylglycine carbamate derivative in moderate yields as shown in Scheme 2.99 The nonsymmetric compound 29 exhibited excellent inhibitory activity for GT-1b. However, it exhibited nanomolar levels of antiviral activity against GT-2a, which indicated no dramatic change in activity.

We also used SuFEx chemistry to prepare the biotin-tagged, biaryl sulfate core based NS5A inhibitor as shown in Scheme 3. Biotin binds specific proteins such as streptavidin and avidin with extremely high affinity. ${ }^{\mathbf{4 4}}$ Because of this specificity, biotinylation of NS5A inhibitors has been reported. ${ }^{\mathbf{1 0 0 , 1 0 1}}$ The introduction of biotin into one proline was readily carried out using SuFex chemistry. Although the NS5A inhibitor containing the biaryl sulfate with biotin-tag 32 showed a slightly decreased $\mathrm{EC}_{50}$ against genotype-1b (9.59 nM), it was 10 times higher than the previously reported biotinylated tag molecules based on BMS compound (BMS-671 and BMS-690). ${ }^{63,102}$ 


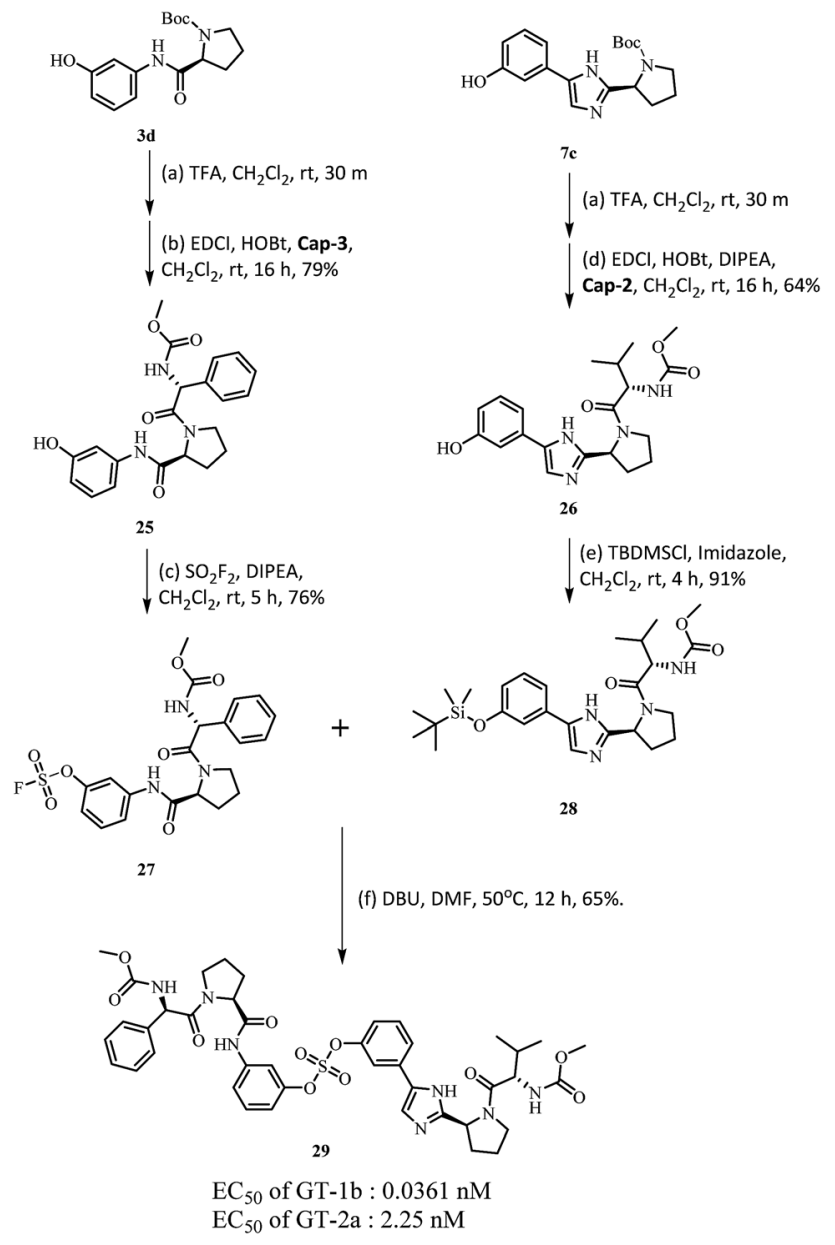

Scheme 2 Synthesis of 3-(2-((S)-1-((methoxycarbonyl)-L-valyl)pyrrolidin-2-yl)-1H-imidazol-5-yl)phenyl(3-((S)-1-((R)-2-((methox-

ycarbonyl)amino)-2-phenylacetyl)pyrrolidine-2-carboxamido)phenyl) sulfate.
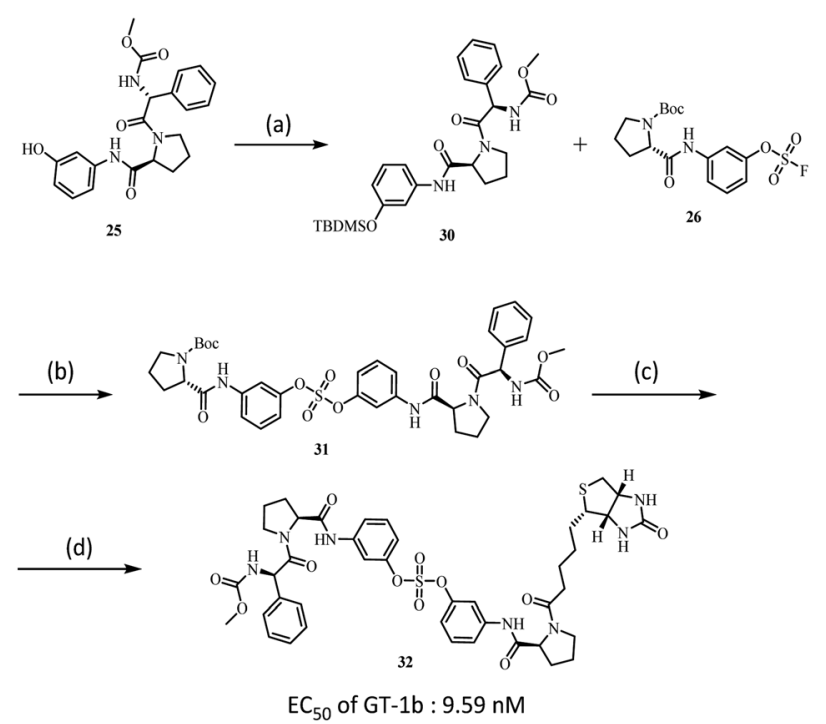

Scheme 3 (a) TBDMSCl, Imidazole, $\mathrm{CH}_{2} \mathrm{Cl}_{2}, \mathrm{rt}, 4 \mathrm{~h}, 81 \%$; (b) DBU, DMF, $50{ }^{\circ} \mathrm{C}, 12$ h, 75\%; (c) TFA, $\mathrm{CH}_{2} \mathrm{Cl}_{2}, \mathrm{rt}, 30$ m; (d) EDCl, HOBt, DIPEA, Biotin, $\mathrm{CH}_{2} \mathrm{Cl}_{2}, \mathrm{rt}, 16 \mathrm{~h}, 96 \%$.
To understand the observed antiviral activities $\left(\mathrm{EC}_{50}\right)$ of inhibitors from a 3D structural perspective, three representative inhibitors, BMK-21007, BMK-21014, and 11, were docked in an NS5A protein dimer model. The model was constructed using the NS5A domain 1 crystal structure $(3 \mathrm{FQQ})^{103}$ and amino terminal alpha helical NMR structure $(1 \mathrm{R} 7 \mathrm{G}){ }^{104}$ Fig. 3 illustrates the $3 \mathrm{D}$ interaction models of BMK-21007, BMK-21014, and 11 with the NS5A dimer. The docking model demonstrates that inhibitors bound across the dimer interface with Thr95A, which forms a hydrogen bond with the nonsymmetric biaryl sulfate core. The interactions of inhibitors and the residues at the binding site are detailed in two dimensional (2D) docking representation (ESI, Fig. S4†). In the case of BMK-21007, the biaryl sulfate moiety can engage in hydrophobic interaction with Gln54A and Gln54B, whereas the methyl groups of the carbamate groups make $\pi$-alkyl interaction with Pro58 and Tyr93 of the NS5A protein dimer (Fig. 3A and S4A $\dagger$ ). The amide linkers of BMK-21014 make hydrogen bond with Gln54, Thr56, and Gln62, whereas both methyl groups of the carbamates involve $\pi$-alkyl interaction with Pro58 and Tyr93 (Fig. 3B and S4B †े). In compound 11 (Fig. 3C), the sulfate moiety appears to interact with Thr95 by hydrogen bonding. Interestingly, Tyr93A has $\pi$-alkyl interaction with the pyrrolidine ring and the methyl moiety of methyl carbamate, whereas Tyr93B shows $\pi$-donor hydrogen bond interaction with the phenyl group of D-phenylglycine. There are no interactions with the residues of Pro58 (ESI, Fig. S4C†).

As shown in a superposed model (Fig. 3D, S4A and S4B $\dagger$ ), the symmetric inhibitors BMK-21007 and BMK-21014 pose reduced steric hindrance of D-phenylglycine moiety compared to nonsymmetrical inhibitor 11, where only one methyl group of the carbamates is involved in $\pi$-alkyl interaction with Tyr93A. Also, CDocker interaction energies of BMK-21007, BMK-21014, and 11 are $-58.41,-57.20$, and $-46.19 \mathrm{kcal} \mathrm{mol}^{-1}$, respectively, which suggests that BMK-21007 and BMK-21014 might be better placed in the binding mode compared to 11. In the case of inhibitor 22, hydrogen bond interactions are found not only between the sulfate and Gly54A but also between the imidazole and Thr56 (ESI,
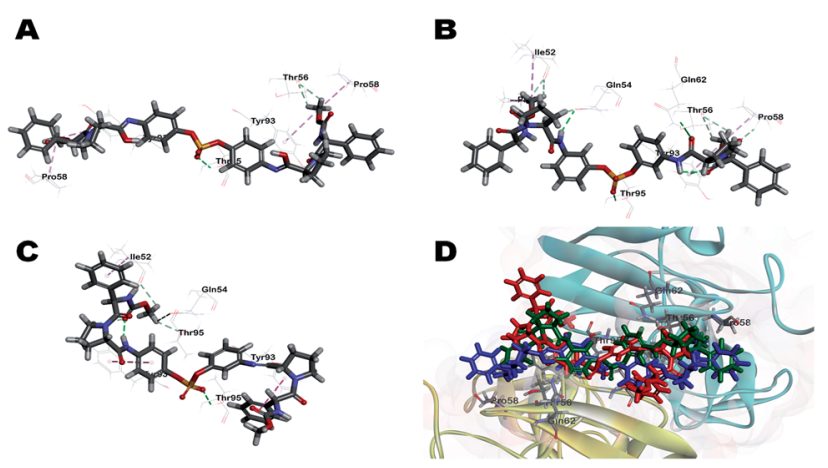

Fig. 3 Docking of (A) BMK-21007, (B) BMK-21014, and (C) 11 to an NS5A (3FQQ) dimer model. For clarity purpose, only residues having interactions were shown. BMK-21007, BMK-21014, and 11 are shown in stick and surrounding residues were shown in line. Various interactions are shown in dashes. (For more clarity see the ESI, Fig. S4†). (D) Superposed model of inhibitors inside 3FQQ dimer model. BMK-21007, BMK21014, and 11 are shown blue, green and red, respectively. 
Fig. S4D $\dagger$ ). Both ends of the carbamate groups appear to show $\pi$ alkyl interaction with Tyr93A and Tyr93B, and especially Tyr93A is engaged in hydrogen bond interaction with the carbonyl moiety of one carbamate. Interestingly, one methyl group of the carbamate makes hydrophobic interaction with Pro58B, whereas the other side correlates with Pro97B. Therefore, the D-phenylglycine of inhibitor 22 poses reduced steric hindrance compared to inhibitor 11 due to the hydrogen bonding of the carbamate group (ESI, Fig. S6 $\dagger$ ). CDocker interaction energy of inhibitor 22 is $-54.76 \mathrm{kcal} \mathrm{mol}^{-1}$, which bodes well with its $\mathrm{EC}_{50}$ activity.

\section{Conclusions}

In conclusion, using SuFEx chemistry we achieved chemoselective coupling of aryl fluorosulfates and aryl silyl ethers in the presence of a catalytic amount of a base. Furthermore, this method was used to synthesize new NS5A inhibitors containing various substituted sulfate core structures with high yields. These compounds exhibited high inhibitory activities against HCV viral proliferation by binding NS5A protein. We easily synthesized inhibitors containing nonsymmetric or $o$-fluoro substituted biaryl sulfate core structures using the SuFEx reaction in a "click chemistry" manner. Furthermore, the monomeric halves of BMK-21014 and BMK21025, which showed excellent activity in our previous report, were easily coupled using SuFEx chemistry to form nonsymmetric biarylsulfate core-based inhibitor 29. Compounds 14, 15, and 29 had two-digit $\mathrm{pM} \mathrm{EC}_{50}$ values against GT-1b and single digit or sub nanomolar values for GT-2a. In addition, a sulfate core-containing compound conjugated with biotin 32 was synthesized in a straightforward manner and showed high activity compared to that of reference biotinylated compounds. Based on these results, SuFEx chemistry proved to be highly useful in constructing symmetric and nonsymmetric sulfate core-containing NS5A inhibitors, and could potentially be applied to the synthesis of a wider range of compounds in medicinal chemistry.

\section{Experimental}

\section{General methods and material}

The ${ }^{1} \mathrm{H},{ }^{13} \mathrm{C}$ and ${ }^{19} \mathrm{~F}$ NMR-spectra were measured with a Varian/ Oxford As-500 (500 MHz), an Agilent 400-MR DD2 Magnetic Resonance System (400 MHz), or Bruker/Avance DPX-300 (300 $\mathrm{MHz}$ ) spectrophotometer. Chemical shifts were measured as part per million ( $d$ values) from Tetramethylsilane (TMS) as an internal standard at probe temperature in $\mathrm{CD}_{3} \mathrm{OD}$ or $\mathrm{CDCl}_{3}$ or DMSO-D $_{6}$ for neutral compounds. Coupling constants are provided in $\mathrm{Hz}$, with the following spectral pattern designations: s, singlet; d, doublet; t, triplet; q, quartet; quint, quintet; m, multiplet; br, broad; app, apparent. Reactions were conducted, purified, and analyzed according to methods widely practiced in the field, while taking necessary precautions in the exclusion of moisture and/or oxygen where appropriate. Evaporation of solvents was performed at reduced pressure using a rotary evaporator. TLC was performed using silica gel 60F254 coated on an aluminium sheet (E. Merck, Art.5554). High resolution mass spectra (HRMS) were recorded on a ThermoFinnigan LCQ $^{\mathrm{TM}}$ Classic, Quadrupole Ion-Trap Mass Spectrometer. HPLC analyses were carried out on an Agilent HP1100 system (Santa Clara, CA, USA), composed of an auto sampler, quaternary pump, photodiode array detector (DAD), and HP Chemstation software. The separation was carried out on a poroshell 120 EC-C18 column $50 \times 4.6 \mathrm{~mm}, 2.7 \mathrm{~mm}$ with acetonitrile (A), $0.1 \%$ TFA in water (B), as a mobile phase at a flow rate of $1 \mathrm{~mL} \mathrm{~min} \mathrm{mit}^{-1}$ at $20{ }^{\circ} \mathrm{C}$. Method: $5 \% \mathrm{~A}$ and $95 \% \mathrm{~B}(0 \mathrm{~min}), 50 \% \mathrm{~A}$ and $50 \% \mathrm{~B}(15$ $\mathrm{min}$ ), 95\% A and 5\% B (24 min), 95\% A and 5\% B (25 min), 5\% A and $95 \% \mathrm{~B}$ (26 min), 5\% A and 95\% B (27 min). All materials were purchased from a commercial supplier and used without further purification unless otherwise noted. The ${ }^{1} \mathrm{H}$ NMR-spectra of intermediates and final products exhibit a mixture of rotamers at ambient temperature in DMSO- $\mathrm{d}_{6}$.

\section{General procedure for synthesis of capping groups ${ }^{64,90,91}$}

$\mathrm{Na}_{2} \mathrm{CO}_{3}(2.6 \mathrm{mmol})$ was added to aq. $\mathrm{NaOH}\left(5 \mathrm{~mL}\right.$ of $1 \mathrm{M} / \mathrm{H}_{2} \mathrm{O}, 5$ $\mathrm{mmol})$ solution of an amino acid $(5.00 \mathrm{mmol})$ and the resulting solution was cooled with ice-water bath. Methyl chloroformate (5.40 mmol) was added dropwise, the cooling bath was removed and the reaction mixture was stirred at ambient temperature for $3.25 \mathrm{~h}$. The reaction mixture was washed with ether $(3 \times 9 \mathrm{~mL})$, and the aqueous phase was cooled with ice-water bath and acidified with concentrated $\mathrm{HCl}$ to a $\mathrm{pH}$ region of 1-2, and extracted with $\mathrm{CH}_{2} \mathrm{Cl}_{2}(3 \times 9 \mathrm{~mL})$. The organic phase was dried $\left(\mathrm{MgSO}_{4}\right)$, filtered, and concentrated in vacuo to afford capping groups as a white solid.

(Methoxycarbonyl)-D-valine (Cap-1). Yield (760 mg, 87\%); ${ }^{1} \mathrm{H}$ NMR (DMSO-d ${ }_{6}, \delta=2.5$ ppm, $300 \mathrm{MHz}$ ): $\delta 12.54(\mathrm{~s}, 1 \mathrm{H}), 7.32(\mathrm{~d}$, $1 \mathrm{H}), 3.84(\mathrm{t}, 1 \mathrm{H}), 3.54(\mathrm{~s}, 3 \mathrm{H}), 2.03(\mathrm{~m}, 1 \mathrm{H}), 0.87$ (d, 6H).

(Methoxycarbonyl)-L-valine (Cap-2). Yield (5.00 g, 86\%); ${ }^{1} \mathrm{H}$ NMR (DMSO-d 6 , $\delta=2.5$ ppm, $400 \mathrm{MHz}$ ): $\delta 12.51$ (br s, 1H), 7.32 $(\mathrm{d}, 1 \mathrm{H}), 3.84(\mathrm{t}, 1 \mathrm{H}), 3.54(\mathrm{~s}, 3 \mathrm{H}), 2.03(\mathrm{~m}, 1 \mathrm{H}), 0.88(\mathrm{~d}, 6 \mathrm{H})$.

(R)-2-((Methoxycarbonyl)amino)-2-phenylacetic acid (Cap-3). Yield (1.40 g, 67\%); ${ }^{1} \mathrm{H}$ NMR (DMSO-d ${ }_{6}, \delta=2.5$ ppm, $500 \mathrm{MHz}$ ): $\delta 12.79$ (br s, 1H), 7.96 (d, 1H), 7.40-7.29 (m, 5H), $5.13(\mathrm{~d}, 1 \mathrm{H})$, $3.55(\mathrm{~s}, 3 \mathrm{H})$.

\section{General procedure for synthesis of aminophenol}

A stirred mixture of nitro phenol $(12.7 \mathrm{mmol})$ in $\mathrm{MeOH}(50 \mathrm{~mL})$ at room temperature was treated with $10 \mathrm{wt} \%$ palladium on charcoal (100 mg). Hydrogen gas was passed through the mixture for $24 \mathrm{~h}$, and then the palladium on charcoal was removed by filtration through Celite. The filtrate was concentrated in vacuo, and was obtained as solid product.

3-Amino-2-fluorophenol (2a). Yield (1.65 g, 99\%); ${ }^{1} \mathrm{H}$ NMR $\left(\mathrm{CD}_{3} \mathrm{OD}, \delta=3.31 \mathrm{ppm}, 400 \mathrm{MHz}\right): \delta 6.69-6.65(\mathrm{t}, 1 \mathrm{H}), 6.34-6.25$ $(\mathrm{m}, 2 \mathrm{H}) ;{ }^{13} \mathrm{C} \mathrm{NMR}\left(\mathrm{CD}_{3} \mathrm{OD}, \delta=49.00 \mathrm{ppm}, 100 \mathrm{MHz}\right): \delta 146.13$, 143.90, 141.59, 124.71, 109.49, 107.95; ${ }^{19} \mathrm{~F}$ NMR $\left(\mathrm{CD}_{3} \mathrm{OD}, 376\right.$ $\mathrm{MHz}$ : $\delta-161.96$; HRMS (ESI) $m / z$ : anal. calcd for $[\mathrm{M}+\mathrm{H}]^{+}$ $\mathrm{C}_{6} \mathrm{H}_{7} \mathrm{FNO}$ : 128.0506; found 128.0482 .

5-Amino-2-fluorophenol (2b). Yield (809 mg, 99\%); ${ }^{1} \mathrm{H}$ NMR $\left(\mathrm{CD}_{3} \mathrm{OD}, \delta=3.31 \mathrm{ppm}, 400 \mathrm{MHz}\right): \delta 6.78-6.73,(\mathrm{t}, 1 \mathrm{H}), 6.34-6.31$ $(\mathrm{q}, 1 \mathrm{H}), 6.17-6.13(\mathrm{~m}, 1 \mathrm{H}) ;{ }^{13} \mathrm{C} \mathrm{NMR}\left(\mathrm{CD}_{3} \mathrm{OD}, \delta=49.00 \mathrm{ppm}\right.$, $100 \mathrm{MHz}): \delta 147.94,145.65,145.15,116.60,107.67,106.22 ;{ }^{19} \mathrm{~F}$ NMR (CD 30 D, $376 \mathrm{MHz}$ ): $\delta-152.91$; HRMS (ESI) $\mathrm{m} / z$ : anal. calcd for $[\mathrm{M}+\mathrm{H}]^{+} \mathrm{C}_{6} \mathrm{H}_{7} \mathrm{FNO}$ : 128.0506; found 128.0508. 


\section{General procedure for synthesis of amide linked intermediate}

A mixture of amino phenol (12.6 mmol), $N$-Boc-L-proline $(3.25 \mathrm{~g}$, $15.1 \mathrm{mmol})$, and EDCI $(2.11 \mathrm{~g}, 16.4 \mathrm{mmol})$ in $\mathrm{CH}_{2} \mathrm{Cl}_{2}(30 \mathrm{~mL})$ was stirred at room temperature for $4 \mathrm{~h}$. The mixture was then poured in $1 \mathrm{~N}$ aq. $\mathrm{HCl}$ solution and Extracted with $\mathrm{CH}_{2} \mathrm{Cl}_{2}$. The organic layer dried over magnesium sulfate, and then was purified by the column chromatography (n-hexane/ethyl acetate). The residue was obtained as solid product.

tert-Butyl

(S)-2-((2-fluoro-3-hydroxyphenyl)carbamoyl)pyrrolidine-1-carboxylate (3a). Yield (3.79 g, 93\%); ${ }^{1} \mathrm{H}$ NMR (DMSO-d ${ }_{6}, \delta=2.5$ ppm, $\left.500 \mathrm{MHz}\right): \delta 9.84(\mathrm{~s}, 1 \mathrm{H}), 9.63(\mathrm{~s}, 1 \mathrm{H}), 7.32-$ $7.21(\mathrm{~m}, 1 \mathrm{H}), 6.90-6.89(\mathrm{~d}, 1 \mathrm{H}), 6.71-6.70(\mathrm{~d}, 1 \mathrm{H}), 4.42-4.33(\mathrm{~m}, 1 \mathrm{H})$, $3.40(\mathrm{~s}, 1 \mathrm{H}), 3.36-3.32(\mathrm{~m}, 1 \mathrm{H}), 2.20-2.12(\mathrm{~m}, 1 \mathrm{H}), 1.87-1.78(\mathrm{~m}, 3 \mathrm{H})$, 1.40-1.31 (app br s, 9H); ${ }^{13} \mathrm{C}$ NMR (DMSO-d 6 , $\delta=39.52$ ppm, 126 $\mathrm{MHz}): \delta 171.90,153.34,145.34,142.79,127.08,123.46,114.31$, 113.46, 78.66, 60.03, 46.67, 31.23, 28.02, 23.43; ${ }^{19}$ F NMR (DMSO-d ${ }_{6}$, $376 \mathrm{MHz}$ ): $\delta-147.91,-149.01$; HRMS (ESI) $\mathrm{m} / z$ : anal. calcd for $[\mathrm{M}+$ $\mathrm{Na}]^{+} \mathrm{C}_{16} \mathrm{H}_{21} \mathrm{FN}_{2} \mathrm{NaO}_{4}$ : 347.1378; found 347.1379.

tert-Butyl (S)-2-((4-fluoro-3-hydroxyphenyl)carbamoyl) pyrrolidine-1-carboxylate (3b). Yield (748 mg, 59\%); ${ }^{1} \mathrm{H}$ NMR (DMSO-d 6 , $\delta=2.5$ ppm, $400 \mathrm{MHz}): \delta 9.83(\mathrm{~s}, 2 \mathrm{H}), 7.38-$ $7.36(\mathrm{~d}, 1 \mathrm{H}), 7.05-7.00(\mathrm{t}, 1 \mathrm{H}), 6.95-6.91(\mathrm{~m}, 1 \mathrm{H}), 4.23-4.13$ (m, $1 \mathrm{H}), 3.44-3.38(\mathrm{~m}, 1 \mathrm{H}), 3.35-3.32(\mathrm{~m}, 1 \mathrm{H}), 2.23-2.12(\mathrm{~m}$, 1H), 1.91-1.75 (m, 3H), 1.39-1.28 (app br s, 9H); ${ }^{13} \mathrm{C}$ NMR $\left(\right.$ DMSO $\left._{6}, \delta=39.52 \mathrm{ppm}, 100 \mathrm{MHz}\right): \delta$ 171.19, 153.11, 148.05, 146.15, 144.59, 135.46, 115.49, 109.99, 109.09, 78.40, $60.30,46.49,30.92,30.09,28.09,27.89,23.87,23.29 ;{ }^{19}$ F NMR (DMSO-d $\left.{ }_{6}, 376 \mathrm{MHz}\right): \delta-141.97 ; \mathrm{HRMS}$ (ESI) $\mathrm{m} / \mathrm{z}$ : anal. calcd for $[\mathrm{M}+\mathrm{Na}]^{+} \mathrm{C}_{16} \mathrm{H}_{21} \mathrm{FN}_{2} \mathrm{NaO}_{4}$ : 347.1378; found 347.1379.

tert-Butyl (S)-2-((4-hydroxyphenyl)carbamoyl)pyrrolidine-1carboxylate (3c). Yield (5.36 g, 87\%). ${ }^{1} \mathrm{H}$ NMR (DMSO-d ${ }_{6}, \delta=$ $2.5 \mathrm{ppm}, 400 \mathrm{MHz}): \delta 9.68(\mathrm{~s}, 1 \mathrm{H}), 9.16(\mathrm{~s}, 1 \mathrm{H}), 7.37-7.34(\mathrm{~d}, 2 \mathrm{H})$, 6.70-6.67 (d, 2H), 4.22-4.12 (m, 1H), 3.43-3.37 (m, 1H), 3.353.29 (m, 1H), 2.19-2.12 (m, 1H), 1.91-1.74 (m, 3H), 1.39-1.28 (app br s, 9H); ${ }^{13} \mathrm{C}$ NMR (DMSO-d $6, \delta=39.52 \mathrm{ppm}, 100 \mathrm{MHz}$ ): $\delta$ 170.76, 153.28, 130.70, 121.02, 115.02, 78.38, 60.27, 46.56, 31.03, 30.23, 27.97, 23.96, 23.39; HRMS (ESI) $\mathrm{m} / \mathrm{z}$ : anal. calcd for $[\mathrm{M}+\mathrm{Na}]^{+} \mathrm{C}_{16} \mathrm{H}_{22} \mathrm{~N}_{2} \mathrm{NaO}_{4}$ : 329.1472; found 329.1472.

tert-Butyl (S)-2-((3-hydroxyphenyl)carbamoyl)pyrrolidine-1carboxylate (3d). Yield (5.41 g, 96\%); ${ }^{1} \mathrm{H}$ NMR $\left(\right.$ DMSO-d $_{6}, \delta=$ $2.5 \mathrm{ppm}, 400 \mathrm{MHz}): \delta 9.83(\mathrm{~s}, 1 \mathrm{H}), 9.36(\mathrm{~s}, 1 \mathrm{H}), 7.18(\mathrm{~s}, 1 \mathrm{H}), 7.08-$ 7.04 (t, 1H), 6.96-6.94 (d, 1H), 6.45-6.43 (d, 1H), 4.24-4.15 (m, $1 \mathrm{H}), 3.37$ (m, 1H), 3.33-3.32 (m, 1H), 2.17-2.08 (m, 1H), 1.861.75 (m, 3H), 1.39-1.27 (app br s, 9H); ${ }^{13} \mathrm{C}$ NMR (DMSO-d 6 , $\delta=$ $39.52 \mathrm{ppm}, 100 \mathrm{MHz}): \delta 171.40,157.63,153.23,140.15,129.28$, 110.38, 110.11, 106.55, 78.49, 60.40, 46.60, 31.06, 27.98, 23.44; HRMS (ESI) $m / z$ : anal. calcd for $[\mathrm{M}+\mathrm{Na}]^{+} \mathrm{C}_{16} \mathrm{H}_{22} \mathrm{~N}_{2} \mathrm{NaO}_{4}$ : 329.1472; found 329.1474.

2-Bromo-1-(4-fluoro-3-hydroxyphenyl)ethan-1-one (5a). A mixture of 4-fluoro-3-hydroxyacetophenone (100 $\mathrm{mg}, 0.649$ mmol), copper(II) bromide (174 $\mathrm{mg}, 0.779 \mathrm{mmol}$ ) in ethyl acetate $(5 \mathrm{~mL})$ /chloroform $(5 \mathrm{~mL})$ was heated and refluxed for $8 \mathrm{~h}$. The mixture was then filtered to remove the copper(I) bromide. The filtrate was concentrated in vacuo, and was obtained as yellow solid $(123 \mathrm{mg}, 81 \%) ;{ }^{1} \mathrm{H}$ NMR $\left(\mathrm{CDCl}_{3}, \delta=\right.$ $7.26 \mathrm{ppm}, 400 \mathrm{MHz}): \delta$ 7.67-7.64 (m, 1H), 7.56-7.51 (m, 1H),
7.19-7.13 (m, 1H), $5.63(\mathrm{~s}, 1 \mathrm{H}), 4.38(\mathrm{~s}, 2 \mathrm{H}) ;{ }^{13} \mathrm{C} \mathrm{NMR}\left(\mathrm{CDCl}_{3}\right.$, $\delta=77.16 \mathrm{ppm}, 100 \mathrm{MHz}): \delta$ 190.18, 155.97, 153.50, 144.29, 131.19, 122.62, 118.44, 116.35, 30.63; ${ }^{19} \mathrm{~F} \mathrm{NMR}\left(\mathrm{CDCl}_{3}, 376\right.$ MHz): -130.70 ; HRMS (ESI) $m / z$ : anal. calcd for $\left[\mathrm{M}+\mathrm{Na}^{+} \mathrm{C}_{8^{-}}\right.$ $\mathrm{H}_{6} \mathrm{BrFNaO}_{2}$ : 254.9427; found 254.9428 .

\section{General procedure for synthesis of keto ester derivatives}

2-Bromo-acetophenone $(0.416 \mathrm{mmol})$ and $N$-Boc-L-proline $(0.499 \mathrm{mmol})$ in acetonitrile $(10 \mathrm{~mL})$ put into the flask. Diisopropylethylamine $(0.541 \mathrm{mmol})$ was added dropwise to the mixture and was stirred at room temperature for $5 \mathrm{~h}$. The mixture was then poured in $1 \mathrm{~N}$ aq. $\mathrm{HCl}$ solution and extracted with $\mathrm{CH}_{2} \mathrm{Cl}_{2}$. The organic layer dried over magnesium sulfate, and then was purified by the column chromatography ( $n$ hexane/ethyl acetate). The residue was obtained as white solid.

1-(tert-Butyl) 2-(2-(4-fluoro-3-hydroxyphenyl)-2-oxoethyl) (S)pyrrolidine-1,2-dicarboxylate (6a). Yield (110 mg, 72\%); ${ }^{1} \mathrm{H}$ NMR (DMSO-d ${ }_{6}, \delta=2.5$ ppm, $400 \mathrm{MHz}$ ): $\delta 10.43(\mathrm{~s}, 1 \mathrm{H}), 7.55-7.48(\mathrm{~m}$, 2H), 7.34-7.29 (m, 1H), 5.56-5.36 (m, 2H), 4.35-4.39/4.08-4.03 $(2 \mathrm{~m}, 0.86 \mathrm{H}+0.28 \mathrm{H}), 3.41-3.34(\mathrm{~m}, 1 \mathrm{H}), 3.28-3.16(\mathrm{~m}, 1 \mathrm{H})$, 2.32-2.09 (m, 1H), 1.92-1.76 (m, 3H), 1.36 (app br s, 9H); ${ }^{13} \mathrm{C}$ NMR (DMSO-d 6 , $\delta=39.52$ ppm, $100 \mathrm{MHz}): \delta$ 191.37, 172.33, $155.97,153.00,145.44,130.79$, 120.36, 116.90, 116.55, 79.06, $66.41,58.56,46.22,30.47,29.55,27.96,23.88,23.12 ;{ }^{19} \mathrm{~F}$ NMR (DMSO-d 6 , $376 \mathrm{MHz}$ ): $\delta-127.51$; HRMS (ESI) $\mathrm{m} / z$ : anal. calcd for $[\mathrm{M}+\mathrm{Na}]^{+} \mathrm{C}_{18} \mathrm{H}_{22} \mathrm{FNNaO}_{6}$ : 390.1323; found 390.1323.

1-(tert-Butyl) 2-(2-(4-hydroxyphenyl)-2-oxoethyl) (S)-pyrrolidine1,2-dicarboxylate (6b). Yield (1.20 g, 74\%); ${ }^{1} \mathrm{H}$ NMR (DMSO-d 6 , $\delta=2.5$ ppm, $400 \mathrm{MHz}): \delta 10.49(\mathrm{~s}, 1 \mathrm{H}), 7.86-7.84$ (d, 2H), 5.52-5.32 (m, 2H), 4.34-4.29 (m, 1H), 3.40-3.37 (m, 1H), 3.32-3.28 (m, 1H), 2.33-2.22 (m, 1H), 2.18-2.12 (m, 1H), 1.91-1.84 (m, 2H), 1.39-1.36 (app br s, 9H); ${ }^{13} \mathrm{C}$ NMR (DMSO-d $6, \delta=39.52 \mathrm{ppm}, 100 \mathrm{MHz}$ ): $\delta$ 190.47, 172.31, 162.68, 152.95, 130.41, 125.35, 115.44, 78.94, $66.11,58.55,46.17,30.43,27.93,23.05$; HRMS (ESI) $m / z:$ anal. calcd for $[\mathrm{M}+\mathrm{Na}]^{+} \mathrm{C}_{18} \mathrm{H}_{23} \mathrm{NNaO}_{6}$ : 372.1418; found 372.1419.

1-(tert-Butyl) 2-(2-(3-hydroxyphenyl)-2-oxoethyl) (S)-pyrrolidine1,2-dicarboxylate (6c). Yield $(1.47 \mathrm{~g}, 91 \%) ;{ }^{1} \mathrm{H}$ NMR (DMSO-d $\mathrm{d}_{6}$, $\delta=2.5 \mathrm{ppm}, 400 \mathrm{MHz}): \delta 9.88(\mathrm{~s}, 1 \mathrm{H}), 7.45-7.43(\mathrm{~d}, 1 \mathrm{H}), 7.37-7.33$ (m, 2H), 7.10-7.08 (d, 1H), 5.58-5.38 (m, 2H), 4.37-4.31 (m, 1H), 3.41-3.31 (m, 2H), 2.34-2.10 (m, 2H), 1.92-1.82 (m, 2H), 1.40-1.37 (app br s, 9H); ${ }^{13} \mathrm{C}$ NMR (DMSO-d $6, \delta=39.52 \mathrm{ppm}, 100 \mathrm{MHz}$ ): $\delta$ 192.42, 172.30, 157.84, 153.00, 135.18, 130.04, 121.13, 118.74, 113.90, 79.00, 66.55, 58.59, 46.21, 30.48, 29.56, 28.11, 27.94, 23.87, 23.12; HRMS (ESI) $m / z$ : anal. calcd for $[\mathrm{M}+\mathrm{Na}]^{+} \mathrm{C}_{18} \mathrm{H}_{23} \mathrm{NNaO}_{6}$ : 372.1418; found 372.1417 .

General procedure for synthesis of imidazole linked intermediate

A mixture of keto ester $(0.554 \mathrm{mmol})$ and ammonium acetate $(8.30 \mathrm{mmol})$ were suspended in toluene $(10 \mathrm{~mL})$. The reaction mixture was heated to $90{ }^{\circ} \mathrm{C}$ and stirred for $20 \mathrm{~h}$. The mixture was then poured in $\mathrm{H}_{2} \mathrm{O}$ and extracted with ethyl acetate. The organic layer dried over magnesium sulfate and evaporated in vacuo. After evaporation, the residue was purified by the column chromatography ( $n$-hexane/ethyl acetate). Then product was obtained as yellow solid. 
tert-Butyl (S)-2-(5-(4-fluoro-3-hydroxyphenyl)-1H-imidazol-2yl)pyrrolidine-1-carboxylate (7a). Yield (101 mg, 52\%); ${ }^{1} \mathrm{H}$ NMR $\left(\right.$ DMSO-d $\left._{6}, \delta=2.5 \mathrm{ppm}, 400 \mathrm{MHz}\right): \delta 12.06 / 11.83-11.76(\mathrm{~s} / \mathrm{d}$, $0.16 \mathrm{H}+0.76 \mathrm{H}), 9.70(\mathrm{~s}, 1 \mathrm{H}), 7.35(\mathrm{~s}, 2 \mathrm{H}), 7.10-7.01(\mathrm{~m}, 2 \mathrm{H})$, 4.80-4.73 (d, 1H), $3.52(\mathrm{~s}, 1 \mathrm{H}), 2.32-2.15(\mathrm{~m}, 1 \mathrm{H}), 1.97-1.76(\mathrm{~m}$, 4H), 1.39-1.15 (app br s, 9H); ${ }^{13} \mathrm{C}$ NMR (DMSO-d $6, \delta=$ $39.52 \mathrm{ppm}, 100 \mathrm{MHz}): \delta$ 153.39, 150.76, 144.58, 138.79, 131.95, 115.97, 115.18, 113.60, 111.31, 78.60, 58.75, 55.20, 46.28, 33.32, 27.88, 23.88, 23.14; ${ }^{19}$ F NMR (DMSO-d 6 , $376 \mathrm{MHz}$ ): $\delta-138.76$, -140.30; HRMS (ESI) $m / z$ : anal. calcd for $[\mathrm{M}+\mathrm{H}]^{+} \mathrm{C}_{18} \mathrm{H}_{23} \mathrm{FN}_{3} \mathrm{O}_{3}$ : 348.1718; found 348.1719.

tert-Butyl (S)-2-(5-(4-hydroxyphenyl)-1H-imidazol-2-yl)pyrrolidine-1-carboxylate (7b). Yield (887 mg, 47\%); ${ }^{1} \mathrm{H}$ NMR (DMSO-d ${ }_{6}, \delta=2.5$ ppm, $\left.400 \mathrm{MHz}\right): \delta 11.76$ (br s, $\left.1 \mathrm{H}\right)$, 9.48 (br s, $1 \mathrm{H}), 7.53(\mathrm{~s}, 2 \mathrm{H}), 7.20(\mathrm{~s}, 1 \mathrm{H}), 6.77-6.75(\mathrm{~d}, 2 \mathrm{H})$, 4.83-4.76 (d, 1H), 3.54-3.35 (d, 2H), 2.20-1.81 (m, 4H), 1.391.17 (app br s, 9H); ${ }^{13} \mathrm{C}$ NMR (DMSO-d $6, \delta=39.52$ ppm, 100 $\mathrm{MHz}): \delta 155.93,153.82,153.47,150.09,125.54,115.30,78.23$, 55.26, 46.34, 33.41, 31.88, 27.93, 23.85, 23.16; HRMS (ESI) $\mathrm{m} /$ $z$ : anal. calcd for $[\mathrm{M}+\mathrm{Na}]^{+} \mathrm{C}_{18} \mathrm{H}_{23} \mathrm{~N}_{3} \mathrm{NaO}_{3}$ : 352.1632; found 352.1634 .

tert-Butyl (S)-2-(5-(3-hydroxyphenyl)-1H-imidazol-2-yl)pyrrolidine-1-carboxylate (7c). Yield (456 mg, 45\%); ${ }^{1} \mathrm{H}$ NMR (DMSO-d $\left.{ }_{6}, \delta=2.5 \mathrm{ppm}, 400 \mathrm{MHz}\right): \delta 11.88$ (br s, 1H), 9.41 (br s, 1H), 7.34-7.11 (m, 4H), 6.60 (s, 1H), 4.85-4.77 (d, $1 \mathrm{H}), 3.55(\mathrm{~s}, 1 \mathrm{H}), 3.35(\mathrm{~s}, 1 \mathrm{H}), 2.22-2.14(\mathrm{~m}, 1 \mathrm{H}), 1.99-1.82$ (m, 3H), 1.39-1.16 (app br s, 9H); ${ }^{13} \mathrm{C}$ NMR (DMSO-d 6 , $\delta=$ $39.52 \mathrm{ppm}, 100 \mathrm{MHz}): \delta$ 157.66, 153.51, 129.43, 115.25, 113.14, 111.25, 78.32, 55.33, 48.72, 46.42, 33.49, 31.93, 27.96, 23.93, 23.25; HRMS (ESI) $m / z$ : anal. calcd for $[\mathrm{M}+\mathrm{Na}]^{+}$ $\mathrm{C}_{18} \mathrm{H}_{23} \mathrm{~N}_{3} \mathrm{NaO}_{3}$ : 352.1632; found 352.1634.

\section{General procedure for synthesis of fosylated intermediate}

A mixture of phenol derivative $(0.800 \mathrm{mmol})$ and $N, N$-diisopropylethylamine $(1.20 \mathrm{mmol})$ in $\mathrm{CH}_{2} \mathrm{Cl}_{2}(5 \mathrm{~mL})$ was stirred under sulfuryl fluoride at room temperature for $5 \mathrm{~h}$. The mixture was then poured in $1 \mathrm{~N}$ aq. $\mathrm{HCl}$ solution and extracted with $\mathrm{CH}_{2} \mathrm{Cl}_{2}$. The organic layer dried over magnesium sulfate, filtered, and concentrated in vacuo. Without any purification, the residue was obtained as an oil.

tert-Butyl (S)-2-((4-((fluorosulfonyl)oxy)phenyl)carbamoyl)pyrrolidine-1-carboxylate (8a). Yield (278 mg, 90\%); ${ }^{1} \mathrm{H}$ NMR $\left(\right.$ DMSO-d $_{6}, \delta=2.5$ ppm, $\left.400 \mathrm{MHz}\right): \delta 10.31(\mathrm{~s}, 1 \mathrm{H}), 7.82-7.80(\mathrm{~d}$, $2 \mathrm{H}), 7.53-7.51(\mathrm{~d}, 2 \mathrm{H}), 4.28-4.21(\mathrm{~m}, 1 \mathrm{H}), 3.42(\mathrm{~s}, 2 \mathrm{H}), 2.21(\mathrm{~s}$, 1H), 1.89-1.79 (m, 3H), 1.38-1.27 (app s, 9H); ${ }^{13} \mathrm{C}$ NMR (DMSO$\left.\mathrm{d}_{6}, \delta=39.52 \mathrm{ppm}, 100 \mathrm{MHz}\right): \delta 171.97,153.13,144.74,139.67$, 121.53, 120.75, 78.57, 60.46, 46.58, 31.01, 30.16, 27.92, 23.99, 23.39; ${ }^{19} \mathrm{~F} \mathrm{NMR}\left(\mathrm{CD}_{3} \mathrm{OD}, 376 \mathrm{MHz}\right): 31.23$; HRMS (ESI) $\mathrm{m} / z$ : anal. calcd for $[\mathrm{M}+\mathrm{Na}]^{+} \mathrm{C}_{16} \mathrm{H}_{21} \mathrm{FN}_{2} \mathrm{NaO}_{6} \mathrm{~S}$ : 411.0997; found 411.1001.

tert-Butyl (S)-2-((3-((fluorosulfonyl)oxy)phenyl)carbamoyl)pyrrolidine-1-carboxylate (8b). Yield (1.66 g, 87\%); ${ }^{1} \mathrm{H}$ NMR (DMSO-d ${ }_{6}, \delta=2.5$ ppm, $\left.400 \mathrm{MHz}\right): \delta 10.43-10.41(\mathrm{~d}, 1 \mathrm{H}), 7.98(\mathrm{~s}$, $1 \mathrm{H}), 7.62-7.59(\mathrm{t}, 1 \mathrm{H}), 7.55-7.50(\mathrm{~m}, 1 \mathrm{H}), 7.26-7.24(\mathrm{~d}, 1 \mathrm{H})$, 4.27-4.17 (m, 1H), 3.46-3.40 (m, 1H), 3.37-3.31 (m, 1H), 2.24$2.16(\mathrm{~m}, 1 \mathrm{H}), 1.94-1.77$ (m, 3H), 1.39-1.25 (app s, 9H); ${ }^{13} \mathrm{C}$ NMR (DMSO-d ${ }_{6}, \delta=39.52$ ppm, $100 \mathrm{MHz}$ ): $\delta$ 172.23, 153.06, 149.63,
$141.01,131.00,119.33,115.30,111.07,78.61,60.48,46.57$, 30.91, 30.11, 27.87, 23.98, 23.39; ${ }^{19} \mathrm{~F}$ NMR ( $\mathrm{CD}_{3} \mathrm{OD}, 376 \mathrm{MHz}$ ): 38.49; HRMS (ESI) $m / z$ : anal. calcd for $[\mathrm{M}+\mathrm{Na}]^{+} \mathrm{C}_{16} \mathrm{H}_{21} \mathrm{FN}_{2}-$ $\mathrm{NaO}_{6} \mathrm{~S}: 411.0997$; found 411.0998.

tert-Butyl (S)-2-((2-fluoro-3-((fluorosulfonyl)oxy)phenyl)carbamoyl)pyrrolidine-1-carboxylate (8c). Yield (364 mg, $83 \%) ;{ }^{1} \mathrm{H}$ NMR (DMSO-d 6 , $\left.\delta=2.5 \mathrm{ppm}, 400 \mathrm{MHz}\right): \delta 10.18(\mathrm{~s}$, $1 \mathrm{H}), 8.04-7.95(\mathrm{~d}, 1 \mathrm{H}), 7.54(\mathrm{~s}, 1 \mathrm{H}), 7.39-7.35(\mathrm{t}, 1 \mathrm{H}), 4.45-$ $4.36(\mathrm{~m}, 1 \mathrm{H}), 3.42(\mathrm{~s}, 1 \mathrm{H}), 3.37-3.33(\mathrm{~m}, 1 \mathrm{H}), 2.23-2.16(\mathrm{~m}$, $1 \mathrm{H}), 1.92-1.80(\mathrm{~s}, 3 \mathrm{H}), 1.40-1.31$ (app s, 9H); ${ }^{13} \mathrm{C} \mathrm{NMR}$ $\left(\right.$ DMSO$\left.-\mathrm{d}_{6}, \delta=39.52 \mathrm{ppm}, 100 \mathrm{MHz}\right): \delta$ 172.20, 153.08, 146.52, 144.00, 136.75, 128.31, 124.97, 118.45, 78.61, 59.87, 46.55, 31.04, 29.94, 27.86, 23.94, 23.30; ${ }^{19}$ F NMR (DMSO-d 6 , $376 \mathrm{MHz}$ ): $\delta$ 39.69, -139.30, -140.16; HRMS (ESI) $\mathrm{m} / \mathrm{z}$ : anal. calcd for $[\mathrm{M}+\mathrm{Na}]^{+} \mathrm{C}_{16} \mathrm{H}_{20} \mathrm{~F}_{2} \mathrm{~N}_{2} \mathrm{NaO}_{6} \mathrm{~S}$ : 429.0902; found 429.0902.

tert-Butyl (S)-2-((4-fluoro-3-((fluorosulfonyl)oxy)phenyl)carbamoyl)pyrrolidine-1-carboxylate (8d). Yield $(260 \mathrm{mg}$, 90\%); ${ }^{1} \mathrm{H}$ NMR (DMSO-d $\left.{ }_{6}, \delta=2.5 \mathrm{ppm}, 400 \mathrm{MHz}\right): \delta 10.42(\mathrm{~s}$, $1 \mathrm{H}), 8.14-8.12(\mathrm{~d}, 1 \mathrm{H}), 7.64-7.56(\mathrm{~m}, 2 \mathrm{H}), 4.24-4.15(\mathrm{~m}, 1 \mathrm{H})$, $3.41(\mathrm{~s}, 1 \mathrm{H}), 3.31(\mathrm{~s}, 1 \mathrm{H}), 2.22-2.17(\mathrm{~m}, 1 \mathrm{H}), 1.93-1.78(\mathrm{~m}$, $3 \mathrm{H}), 1.39-1.25$ (app s, 9H); ${ }^{13} \mathrm{C}$ NMR $\left(\right.$ DMSO-d $_{6}, \delta=$ $39.52 \mathrm{ppm}, 100 \mathrm{MHz}): \delta$ 172.14, 153.63, 153.05, 149.36, 146.90, 136.55, 135.74, 135.60, 120.93, 118.14, 113.36, 78.65, $60.46,46.59,30.92,30.11,27.90,24.00,23.40 ;{ }^{19} \mathrm{~F}$ NMR (DMSO-d ${ }_{6}, 376 \mathrm{MHz}$ ): $\delta 39.41,-136.14$; HRMS (ESI) $\mathrm{m} / z$ : anal. calcd for $[\mathrm{M}+\mathrm{Na}]^{+} \mathrm{C}_{16} \mathrm{H}_{20} \mathrm{~F}_{2} \mathrm{~N}_{2} \mathrm{NaO}_{6} \mathrm{~S}$ : 429.0902; found 429.0900 .

tert-Butyl (S)-2-(5-(3-((fluorosulfonyl)oxy)phenyl)-1H-imidazol2-yl)pyrrolidine-1-carboxylate (8e). Yield (49.8 mg, 80\%); ${ }^{1} \mathrm{H}$ NMR (DMSO-d ${ }_{6}, \delta=2.5$ ppm, $400 \mathrm{MHz}$ ): $\delta 8.31-8.25$ (d, 2H), 8.14-8.10 $(\mathrm{t}, 1 \mathrm{H}), 7.74-7.70(\mathrm{t}, 1 \mathrm{H}), 7.63-7.61(\mathrm{~d}, 1 \mathrm{H}), 5.17-5.13(\mathrm{t}, 1 \mathrm{H}), 3.66-$ $3.61(\mathrm{~m}, 1 \mathrm{H}), 3.44-3.37(\mathrm{~m}, 1 \mathrm{H}), 2.43-2.34(\mathrm{~m}, 1 \mathrm{H}), 2.11-1.90(\mathrm{~m}$, $3 \mathrm{H}), 1.38-1.16$ (app s, 9H); ${ }^{13} \mathrm{C}$ NMR $\left(\right.$ DMSO-d $_{6}, \delta=39.52 \mathrm{ppm}$, $100 \mathrm{MHz}): \delta$ 153.82, 152.56, 150.85, 150.11, 131.82, 130.54, 125.80, 121.20, 117.86, 116.57, 79.34, 53.31, 46.54, 32.94, 27.73, 23.45, 17.96, 16.69, 12.20. ${ }^{19}$ F NMR (DMSO-d 6 , 376 MHz): 38.53; HRMS (ESI) $m / z$ : anal. calcd for $[\mathrm{M}+\mathrm{H}]^{+} \mathrm{C}_{18} \mathrm{H}_{23} \mathrm{FN}_{3} \mathrm{O}_{5} \mathrm{~S}$ : 412.1337; found 412.1339.

tert-Butyl (S)-2-(5-(4-fluoro-3-((fluorosulfonyl)oxy)phenyl)$\mathbf{1 H}$-imidazol-2-yl)pyrrolidine-1-carboxylate (8f). Yield $(59.6 \mathrm{mg}$, 92\%); ${ }^{1} \mathrm{H}$ NMR (DMSO-d ${ }_{6}, \delta=2.5$ ppm, $400 \mathrm{MHz}$ ): $\delta 12.07-12.00$ $(\mathrm{d}, 1 \mathrm{H}), 8.03-8.01(\mathrm{~d}, 1 \mathrm{H}), 7.91-7.87(\mathrm{~m}, 1 \mathrm{H}), 7.66-7.64(\mathrm{~m}, 1 \mathrm{H})$, 7.58-7.54 (t, 1H), 4.82-4.77 (d, 1H), $3.53(\mathrm{~s}, 1 \mathrm{H}), 3.34-3.29(\mathrm{~m}$, $1 \mathrm{H}), 2.22$ (s, 1H), 1.96-1.85 (m, 3H), 1.39-1.15 (app s, 9H); ${ }^{13} \mathrm{C}$ NMR (DMSO-d 6 , $\delta=39.52$ ppm, $100 \mathrm{MHz}): \delta$ 153.36, 151.81, 151.06, 149.33, 136.79, 133.63, 125.85, 118.19, 113.46, 78.26, $55.23,46.31,33.29,31.84,27.86,23.83,23.10 ;{ }^{19}$ F NMR (DMSO$\mathrm{d}_{6}, 376 \mathrm{MHz}$ ): $\delta 39.85,-134.19$; HRMS (ESI) $\mathrm{m} / \mathrm{z}$ : anal. calcd for $[\mathrm{M}+\mathrm{H}]^{+} \mathrm{C}_{18} \mathrm{H}_{22} \mathrm{~F}_{2} \mathrm{~N}_{3} \mathrm{O}_{5} \mathrm{~S}$ : 430.1243; found 430.1243.

tert-Butyl (S)-2-(5-(4-((fluorosulfonyl)oxy)phenyl)-1H-imidazol2-yl)pyrrolidine-1-carboxylate (8g). Yield (100 mg, 80\%); ${ }^{1} \mathrm{H}$ NMR (DMSO-d ${ }_{6}, \delta=2.5$ ppm, $500 \mathrm{MHz}$ ): $\delta 12.50$ (s, 1H), $7.93-7.91$ (d, $2 \mathrm{H}), 7.68-7.64(\mathrm{~d}, 1 \mathrm{H}), 7.57-7.56(\mathrm{~d}, 2 \mathrm{H}), 4.88-4.79(\mathrm{~m}, 1 \mathrm{H}), 3.56-$ $3.53(\mathrm{t}, 1 \mathrm{H}), 3.39-3.35(\mathrm{~m}, 1 \mathrm{H}), 2.28-2.17$ (m, 1H), 1.99-1.85 (m, 3H), 1.39-1.15 (app s, 9H); ${ }^{13} \mathrm{C}$ NMR (DMSO-d 6 , $\delta=39.52 \mathrm{ppm}$, $126 \mathrm{MHz}$ ): $\delta$ 153.22, 151.07, 147.88, 126.11, 121.25, 78.35, 54.90, 
46.32, 33.23, 31.81, 28.15, 27.83, 23.82, 23.12; ${ }^{19}$ F NMR (DMSO$\mathrm{d}_{6}, 376 \mathrm{MHz}$ ): 38.27; HRMS (ESI) $\mathrm{m} / z$ : anal. calcd for $[\mathrm{M}+\mathrm{H}]^{+}$ $\mathrm{C}_{18} \mathrm{H}_{23} \mathrm{FN}_{3} \mathrm{O}_{5} \mathrm{~S}$ : 412.1337; found 412.1340.

\section{General procedure for silyl protection}

A mixture of phenol derivative $(2.29 \mathrm{mmol})$, tert-butyldimethylsilyl chloride $(3.44 \mathrm{mmol})$, imidazole $(6.87 \mathrm{mmol})$ in THF $(10 \mathrm{~mL})$ was stirred at room temperature for $4 \mathrm{~h}$. The mixture was then poured in $\mathrm{H}_{2} \mathrm{O}$ and extracted with $\mathrm{CH}_{2} \mathrm{Cl}_{2}$. The organic layer dried over magnesium sulfate, and then was purified by the column chromatography (n-hexane/ethyl acetate). The residue was obtained as sticky oil.

tert-Butyl (S)-2-((3-((tert-butyldimethylsilyl)oxy)phenyl)carbamoyl)pyrrolidine-1-carboxylate (9a). Yield (759 mg, 95\%); ${ }^{1} \mathrm{H}$ NMR (DMSO-d $\left.6=2.5 \mathrm{ppm}, 400 \mathrm{MHz}\right): \delta 9.93(\mathrm{~s}$, $1 \mathrm{H}), 7.35-7.24(\mathrm{~d}, 1 \mathrm{H}), 7.16-7.09(\mathrm{~m}, 2 \mathrm{H}), 6.52-6.51(\mathrm{~d}, 1 \mathrm{H})$, 4.23-4.13 (m, 1H), 3.44-3.38 (m, 1H), 3.32-3.30 (m, 1H), 2.21$2.12(\mathrm{~m}, 1 \mathrm{H}), 1.90-1.75(\mathrm{~m}, 3 \mathrm{H}), 1.39-1.26$ (app s, 9H), 0.93 (s, 9H), 0.17 (s, 6H); ${ }^{13} \mathrm{C}$ NMR (DMSO-d $6, \delta=39.52$ ppm, 100 $\mathrm{MHz}): \delta 171.50,155.27,153.11,140.22,129.48,114.63$, 112.27, 110.84, 78.44, 60.40, 46.56, 30.95, 27.89, 25.55, 23.43, 17.95, -4.53; HRMS (ESI) $m / z$ : anal. calcd for $[\mathrm{M}+\mathrm{Na}]^{+} \mathrm{C}_{22^{-}}$ $\mathrm{H}_{36} \mathrm{~N}_{2} \mathrm{NaO}_{4} \mathrm{Si}$ : 443.2337; found 443.2337.

tert-Butyl (S)-2-((3-((tert-butyldimethylsilyl)oxy)-4-fluorophenyl)carbamoyl)pyrrolidine-1-carboxylate (9b). Yield (318 mg, 73\%); ${ }^{1} \mathrm{H}$ NMR (DMSO-d 6 , $\delta=2.5 \mathrm{ppm}, 400 \mathrm{MHz}$ ): $\delta 9.95(\mathrm{~s}, 1 \mathrm{H}), 7.51-7.38(\mathrm{q}, 1 \mathrm{H}), 7.09(\mathrm{~s}, 2 \mathrm{H}), 4.22-4.12(\mathrm{~m}$, $1 \mathrm{H}), 3.42-3.39(\mathrm{~m}, 1 \mathrm{H}), 3.35-3.30(\mathrm{~m}, 1 \mathrm{H}), 2.21-2.12(\mathrm{~m}, 1 \mathrm{H})$, 1.87-1.75 (m, 3H), 1.39-1.25 (app br s, 9H), 0.95 (s, 9H), 0.17 $(\mathrm{s}, 6 \mathrm{H}) ;{ }^{13} \mathrm{C}$ NMR (DMSO-d $6, \delta=39.52 \mathrm{ppm}, 100 \mathrm{MHz}$ ): $\delta 171.38,153.08,150.51,148.14,142.08,135.72,115.92$, 113.02, 78.41, 60.40, 46.55, 30.94, 27.85, 25.34, 23.42, 17.95, $-4.89 ;{ }^{19} \mathrm{~F}$ NMR (DMSO-d 6 , $376 \mathrm{MHz}$ ): $\delta-138.76$; HRMS (ESI) $m / z$ : anal. calcd for $[\mathrm{M}+\mathrm{Na}]^{+} \mathrm{C}_{22} \mathrm{H}_{35} \mathrm{FN}_{2} \mathrm{NaO}_{4} \mathrm{Si}$ : 461.2242; found 461.2242 .

tert-Butyl (S)-2-((3-((tert-butyldimethylsilyl)oxy)-2-fluorophenyl)carbamoyl)pyrrolidine-1-carboxylate (9c). Yield (294 mg, 73\%); ${ }^{1} \mathrm{H}$ NMR (DMSO-d $6, \delta=2.5 \mathrm{ppm}, 400 \mathrm{MHz}$ ): $\delta 9.71(\mathrm{~s}, 1 \mathrm{H}), 7.54-7.38(\mathrm{~m}, 1 \mathrm{H}), 7.02-6.98(\mathrm{t}, 1 \mathrm{H}), 6.78-6.74$ $(\mathrm{t}, 1 \mathrm{H}), 4.40-4.33(\mathrm{~m}, 1 \mathrm{H}), 3.43-3.38(\mathrm{~m}, 1 \mathrm{H}), 2.24-2.08(\mathrm{~m}$, $1 \mathrm{H}), 1.91-1.77$ (m, 3H), 1.40-1.30 (app br s, 9H), 0.96 (s, 9H), $0.17(\mathrm{~s}, 6 \mathrm{H}) ;{ }^{13} \mathrm{C}$ NMR (DMSO-d ${ }_{6}, \delta=39.52 \mathrm{ppm}, 100 \mathrm{MHz}$ ): $\delta 171.82,153.14,142.85,127.23,123.61,123.56,117.48$, 116.90, 78.51, 59.85, 46.54, 31.05, 27.88, 25.38, 23.32, 18.00, $-4.84 ;{ }^{19} \mathrm{~F}$ NMR (DMSO-d $\left.6,376 \mathrm{MHz}\right): \delta-143.28,-144.70$; HRMS (ESI) $m / z$ : anal. calcd for $[\mathrm{M}+\mathrm{Na}]^{+} \mathrm{C}_{22} \mathrm{H}_{35} \mathrm{FN}_{2} \mathrm{NaO}_{4} \mathrm{Si}$ : 461.2242; found 461.2242.

tert-Butyl (S)-2-(5-(4-((tert-butyldimethylsilyl)oxy)phenyl)1H-imidazol-2-yl)pyrrolidine-1-carboxylate (9d). Yield $(269 \mathrm{mg}$, $60 \%) ;{ }^{1} \mathrm{H}$ NMR (DMSO-d $\left.{ }_{6}, \delta=2.5 \mathrm{ppm}, 500 \mathrm{MHz}\right): \delta 11.77(\mathrm{~s}$, 1H), 7.60-7.59 (d, 2H), 7.30 (s, 1H), 6.81-6.80 (d, 2H), 4.83-4.75 $(\mathrm{m}, 1 \mathrm{H}), 3.56-3.50(\mathrm{~m}, 1 \mathrm{H}), 3.37-3.34(\mathrm{~m}, 1 \mathrm{H}), 2.23-2.12(\mathrm{~m}$, 1H), 2.03-1.83 (m, 3H), 1.39-1.16 (app br s, 9H), 0.95 (s, 9H), $0.18(\mathrm{~s}, 6 \mathrm{H}) ;{ }^{13} \mathrm{C}$ NMR (DMSO-d 6 , $\delta=39.52 \mathrm{ppm}, 126 \mathrm{MHz}$ ): $\delta 153.70,153.37,139.36,128.82,125.44,119.76,78.12,55.20$, $46.25,33.30,31.76,27.88,25.57,23.79,23.05,17.94,-3.21$,
-4.52; HRMS (ESI) $m / z$ : anal. calcd for $[\mathrm{M}+\mathrm{H}]+\mathrm{C}_{24} \mathrm{H}_{38} \mathrm{~N}_{3} \mathrm{O}_{3} \mathrm{Si}$ : 444.2677; found 444.2679.

tert-Butyl (S)-2-(5-(3-((tert-butyldimethylsilyl)oxy)-4-fluorophenyl)-1H-imidazol-2-yl)pyrrolidine-1-carboxylate (9e). Yield (638 mg, 69\%); ${ }^{1} \mathrm{H}$ NMR (DMSO-d ${ }_{6}, \delta=2.5$ ppm, $400 \mathrm{MHz}$ ): $\delta 12.12-11.81(\mathrm{~m}, 1 \mathrm{H}), 7.43(\mathrm{~s}, 1 \mathrm{H}), 7.35-7.31(\mathrm{~m}, 2 \mathrm{H}), 7.23-7.10$ $(\mathrm{m}, 1 \mathrm{H}), 4.83-4.76(\mathrm{~m}, 1 \mathrm{H}), 3.53(\mathrm{~s}, 1 \mathrm{H}), 2.21-2.10(\mathrm{~m}, 1 \mathrm{H}), 1.98-$ $1.84(\mathrm{~m}, 3 \mathrm{H}), 1.39$ (s, 4H), $1.15(\mathrm{~s}, 9 \mathrm{H}), 0.97(\mathrm{~s}, 9 \mathrm{H}), 0.18(\mathrm{~s}, 6 \mathrm{H})$; ${ }^{13} \mathrm{C}$ NMR (DMSO- $\left.\mathrm{d}_{6}, \delta=39.52 \mathrm{ppm}, 100 \mathrm{MHz}\right): \delta 153.10,150.70$, 142.40, 138.33, 132.30, 118.02, 117.76, 116.26, 116.07, 111.83, $78.15,55.30,46.25,33.25,31.81,27.84,25.42,23.73,23.06$, 18.01, -4.82; ${ }^{19} \mathrm{~F}$ NMR (DMSO-d 6 , $376 \mathrm{MHz}$ ): $\delta-134.91$, -136.21; HRMS (ESI) $m / z$ : anal. calcd for $[\mathrm{M}+\mathrm{H}]^{+} \mathrm{C}_{24} \mathrm{H}_{37} \mathrm{FN}_{3^{-}}$ $\mathrm{O}_{3} \mathrm{Si}$ : 462.2583; found 462.2585.

tert-Butyl (S)-2-((4-((tert-butyldimethylsilyl)oxy)phenyl)carbamoyl)pyrrolidine-1-carboxylate (9f). Yield $(63.0 \mathrm{mg}$, 92\%); ${ }^{1} \mathrm{H}$ NMR (DMSO-d ${ }_{6}, \delta=2.5 \mathrm{ppm}, 500 \mathrm{MHz}$ ): $\delta 9.83-9.81$ $(\mathrm{d}, 1 \mathrm{H}), 7.47-7.44(\mathrm{~m}, 2 \mathrm{H}), 6.79-6.78(\mathrm{~d}, 2 \mathrm{H}), 4.22-4.13(\mathrm{~m}$, $1 \mathrm{H}), 3.43-3.40(\mathrm{~m}, 1 \mathrm{H}), 3.32-3.30(\mathrm{~m}, 1 \mathrm{H}), 2.20-2.13(\mathrm{~m}, 1 \mathrm{H})$, 1.91-1.75 (m, 3H), 1.39-1.27 (app s, 9H), 0.94 (s, 9H), 0.16 (s, $6 \mathrm{H}) ;{ }^{13} \mathrm{C}$ NMR (DMSO-d $\left.{ }_{6}, \delta=39.52 \mathrm{ppm}, 126 \mathrm{MHz}\right): \delta 170.98$, $153.16,150.73$, 132.92, 120.77, 119.73, 78.40, 60.29, 46.55, 31.02, 27.95, 25.58, 23.96, 23.37, 17.93; HRMS (ESI) $m / z$ : anal. calcd for $[\mathrm{M}+\mathrm{Na}]^{+} \mathrm{C}_{22} \mathrm{H}_{36} \mathrm{~N}_{2} \mathrm{NaO}_{4} \mathrm{Si}$ : 443.2337; found 443.2340 .

tert-Butyl (S)-2-(5-(3-((tert-butyldimethylsilyl)oxy)phenyl)$1 \mathrm{H}$-imidazol-2-yl)pyrrolidine-1-carboxylate (9g). Yield (545 mg, 81\%); ${ }^{1} \mathrm{H}$ NMR (DMSO-d 6 , $\delta=2.5$ ppm, $500 \mathrm{MHz}$ ): $\delta 11.88-11.82$ (app s, 1H), 7.44-7.18 (m, 4H), $6.63(\mathrm{~s}, 1 \mathrm{H}), 4.83-4.77(\mathrm{~d}, 1 \mathrm{H}), 3.54$ $(\mathrm{s}, 1 \mathrm{H}), 3.36(\mathrm{~s}, 1 \mathrm{H}), 2.22-2.14(\mathrm{~d}, 1 \mathrm{H}), 2.07(\mathrm{~s}, 1 \mathrm{H}), 1.97-1.83(\mathrm{~m}$, 2H), 1.39-1.16 (app s, 9H), 0.96 (s, 9H), 0.19 (s, 6H); ${ }^{13} \mathrm{C}$ NMR (DMSO-d ${ }_{6}, \delta=39.52$ ppm, $126 \mathrm{MHz}$ ): $\delta$ 155.26, 153.39, 139.10, 129.35, 117.52, 115.55, 112.01, 78.10, 55.27, 46.26, 33.27, 31.79, 30.63, 27.84, 25.57, 23.74, 23.08, 17.93; HRMS (ESI) $m / z$ : anal. calcd for $[\mathrm{M}+\mathrm{H}]^{+} \mathrm{C}_{24} \mathrm{H}_{38} \mathrm{~N}_{3} \mathrm{O}_{3}$ Si: 444.2677; found 444.2681.

\section{General procedure for SuFEx reaction}

A mixture of silylated monomer $(0.408 \mathrm{mmol})$, fosylated monomer $(0.272 \mathrm{mmol})$ and DBU $(0.05 \mathrm{mmol})$ in DMF $(10 \mathrm{~mL})$ was stirred at $50{ }^{\circ} \mathrm{C}$ for $12 \mathrm{~h}$. The mixture was then poured in $1 \mathrm{~N}$ aq. $\mathrm{HCl}$ solution and extracted with $\mathrm{CH}_{2} \mathrm{Cl}_{2}$. The organic layer dried over magnesium sulfate, and then was purified by the column chromatography ( $n$-hexane/ethyl acetate). The residue was obtained as solid.

Di-tert-butyl 2,2'-((((sulfonylbis(oxy))bis(4,1-phenylene))-bis(azanediyl))bis(carbonyl))(2S,2'S)-bis(pyrrolidine-1-carboxylate) (10a). Yield (76.1 mg, 90\%); ${ }^{1} \mathrm{H}$ NMR (DMSO-d $\left.{ }_{6}, \delta=2.5 \mathrm{ppm}, 400 \mathrm{MHz}\right)$ : $\delta 10.28(\mathrm{~s}, 1 \mathrm{H}), 7.72-7.70(\mathrm{~d}, 4 \mathrm{H}), 7.39-7.37(\mathrm{~d}, 4 \mathrm{H}), 4.23-4.16(\mathrm{~m}$, 2H), 3.39-3.32 (m, 4H), 2.21-2.16 (t, 2H), 1.86-1.78 (m, 6H), 1.381.25 (app br s, $18 \mathrm{H}$ ); ${ }^{13} \mathrm{C}$ NMR (DMSO-d $6, \delta=39.52 \mathrm{ppm}, 100$ MHz): $\delta 172.2$, 153.5, 145.2, 138.8, 121.9, 120.9, 79.0, 63.0, 60.7, 46.8, 31.2, 28.2, 23.7; HRMS (ESI) $m / z$ : anal. calcd for $[\mathrm{M}+\mathrm{Na}]^{+}$ $\mathrm{C}_{32} \mathrm{H}_{42} \mathrm{~N}_{4} \mathrm{NaO}_{10} \mathrm{~S}$ : 697.2514; found 697.2515.

Di-tert-butyl 2,2'-((((sulfonylbis(oxy))bis(3,1-phenylene))-bis(azanediyl))bis(carbonyl))(2S,2'S)-bis(pyrrolidine-1-carboxylate) (10b). Yield (1.84 g, 69\%); ${ }^{1} \mathrm{H}$ NMR (DMSO-d $6, \delta=2.5$ ppm, $400 \mathrm{MHz}$ ): 
$\delta 10.32(\mathrm{~s}, 1 \mathrm{H}), 8.21-7.77(\mathrm{~m}, 3 \mathrm{H}), 7.59-7.57(\mathrm{~d}, 2 \mathrm{H}), 7.47(\mathrm{~s}, 1 \mathrm{H})$, 7.40-7.22 (m, 1H), 7.12-7.08 (t, 1H), 4.24-4.18 (d, 2H), 3.41 (br s, 4H), 2.20 (s, 2H), 1.88-1.80 (m, 6H), 1.39-1.24 (app br s, 18H); ${ }^{13} \mathrm{C}$ NMR (DMSO-d ${ }_{6}, \delta=39.52 \mathrm{ppm}, 100 \mathrm{MHz}$ ): $\delta$ 172.0, 153.1, 149.9, 140.8, 130.6, 118.4, 115.3, 111.4, 78.6, 60.5, 46.6, 30.9, 27.9, 23.4; HRMS (ESI) $m / z$ : anal. calcd for $[\mathrm{M}+\mathrm{Na}]^{+} \mathrm{C}_{32} \mathrm{H}_{42} \mathrm{~N}_{4} \mathrm{NaO}_{10} \mathrm{~S}$ : 697.2514; found 697.2514 .

tert-Butyl $\quad(S)-2-((3-)((4-)(S)-1-($ tert-butoxycarbonyl) pyrrolidine-2-carboxamido)phenoxy)-sulfonyl)oxy)phenyl)carbamoyl)pyrrolidine-1-carboxylate (10c). Yield $(170 \mathrm{mg}$, 93\%); ${ }^{1} \mathrm{H}$ NMR (DMSO-d $\left.6, \delta=2.5 \mathrm{ppm}, 500 \mathrm{MHz}\right): \delta 10.34-$ $10.23(\mathrm{~d}, 2 \mathrm{H}), 7.96-7.88(\mathrm{~m}, 1 \mathrm{H}), 7.75-7.73(\mathrm{~d}, 2 \mathrm{H}), 7.58-7.55$ $(\mathrm{t}, 1 \mathrm{H}), 7.49-7.37(\mathrm{~m}, 3 \mathrm{H}), 7.11-7.10(\mathrm{~d}, 1 \mathrm{H}), 4.26-4.16(\mathrm{~m}$, $2 \mathrm{H}), 3.45-3.40(\mathrm{~m}, 2 \mathrm{H}), 3.36-3.31(\mathrm{~m}, 2 \mathrm{H}), 2.23-2.15(\mathrm{~m}, 2 \mathrm{H})$, 1.93-1.77 (m, 6H), 1.39-1.24 (app br s, $18 \mathrm{H})$; ${ }^{13} \mathrm{C}$ NMR $\left(\right.$ DMSO-d $\left._{6}, \delta=39.52 \mathrm{ppm}, 126 \mathrm{MHz}\right): \delta 171.80,153.08$, 149.91, 144.95, 144.91, 140.75, 138.75, 130.59, 121.56, 120.58, 118.31, 115.45, 111.39, 78.54, 60.41, 46.58, 30.95, 30.14, 27.90, 23.99, 23.40; HRMS (ESI) $m / z$ : anal. calcd for $[\mathrm{M}+\mathrm{Na}]^{+}$ $\mathrm{C}_{32} \mathrm{H}_{42} \mathrm{~N}_{4} \mathrm{NaO}_{10} \mathrm{~S}$ : 697.2514; found 697.2515.

Di-tert-butyl 2,2'-((((sulfonylbis(oxy))bis(4-fluoro-3,1-phenylene))bis(azanediyl))bis(carbonyl))(2S,2'S)-bis(pyrrolidine-1-carboxylate) (10d). Yield (217 mg, 78\%); ${ }^{1} \mathrm{H}$ NMR (DMSO-d $\mathrm{d}_{6}, \delta=$ $2.5 \mathrm{ppm}, 400 \mathrm{MHz}): \delta 10.33(\mathrm{~s}, 2 \mathrm{H}), 8.11-8.08(\mathrm{t}, 2 \mathrm{H}), 7.60-7.58(\mathrm{~d}$, $2 \mathrm{H})$, 7.53-7.48 (t, 2H), 4.23-4.13 (m, 2H), 3.42-3.40 (m, 2H), 3.35$3.32(\mathrm{~m}, 2 \mathrm{H}), 2.21-2.18(\mathrm{~m}, 2 \mathrm{H}), 1.91-1.78$ (m, 6H), 1.38-1.23 (app br s, $18 \mathrm{H}$ ); ${ }^{13} \mathrm{C}$ NMR (DMSO-d $6, \delta=39.52 \mathrm{ppm}, 100 \mathrm{MHz}$ ): $\delta 171.92,153.60,153.04,149.87,147.41,136.27,119.94,117.90$, $113.55,78.60,60.45,46.57,30.92,30.11,27.86,23.99,23.40 ;{ }^{19} \mathrm{~F}$ NMR (DMSO-d $6376 \mathrm{MHz}$ ): $\delta$-135.44; HRMS (ESI) $m / z$ : anal. calcd for $[\mathrm{M}+\mathrm{Na}]^{+} \mathrm{C}_{32} \mathrm{H}_{40} \mathrm{~F}_{2} \mathrm{~N}_{4} \mathrm{NaO}_{10} \mathrm{~S}$ : 733.2325; found 733.2324.

Di-tert-butyl 2,2'-((((sulfonylbis(oxy))bis(2-fluoro-3,1-phenylene))bis(azanediyl))bis(carbonyl))(2S,2'S)-bis(pyrrolidine-1-carboxylate) (10e). Yield (231 mg, 98\%); ${ }^{1} \mathrm{H}$ NMR (DMSO- $\mathrm{d}_{6}, \delta=$ $2.5 \mathrm{ppm}, 400 \mathrm{MHz}): \delta 10.12(\mathrm{~s}, 2 \mathrm{H}), 8.00-7.97(\mathrm{t}, 1 \mathrm{H}), 7.90-7.87(\mathrm{t}$, $1 \mathrm{H}), 7.43-7.40(\mathrm{t}, 2 \mathrm{H}), 7.35-7.31(\mathrm{t}, 2 \mathrm{H}), 4.43-4.34(\mathrm{~m}, 2 \mathrm{H}), 3.44-$ $3.39(\mathrm{~m}, 2 \mathrm{H}), 3.30(\mathrm{~m}, 2 \mathrm{H}), 2.32-2.11(\mathrm{~m}, 2 \mathrm{H}), 1.91-1.76(\mathrm{~m}, 6 \mathrm{H})$, 1.40-1.29 (br s, $18 \mathrm{H}$ ); ${ }^{13} \mathrm{C}$ NMR (DMSO-d ${ }_{6}, v=39.52$ ppm, 100 $\mathrm{MHz}): \delta 172.17,153.07,137.06,128.08,124.67,124.04,118.49$, 78.61, 59.83, 46.55, 31.04, 29.98, 27.90, 23.95, 23.31; ${ }^{19} \mathrm{~F}$ NMR (DMSO-d 6 , 376 MHz): $\delta-138.71,-139.67$; HRMS (ESI) $m / z$ : anal. calcd for $[\mathrm{M}+\mathrm{Na}]^{+} \mathrm{C}_{32} \mathrm{H}_{40} \mathrm{~F}_{2} \mathrm{~N}_{4} \mathrm{NaO}_{10} \mathrm{~S}$ : 733.2325; found 733.2321.

tert-Butyl (S)-2-((3-)((5- $((S)-1-($ tert-butoxycarbonyl)pyrrole-dine2-carboxamido)-2-fluorophenoxy)sulfonyl)oxy)-2-fluoro-phenyl)carbamoyl)pyrrolidine-1-carboxylate (10f). Yield (112 mg, 58\%); ${ }^{1} \mathrm{H}$ NMR (DMSO-d $\left.{ }_{6}, \delta=2.5 \mathrm{ppm}, 400 \mathrm{MHz}\right): \delta 10.33(\mathrm{~s}, 1 \mathrm{H}), 10.11(\mathrm{~s}$, $1 \mathrm{H}), 8.12-7.90(\mathrm{~m}, 2 \mathrm{H}), 7.61(\mathrm{~s}, 1 \mathrm{H}), 7.54-7.49(\mathrm{t}, 1 \mathrm{H}), 7.40-7.31(\mathrm{~m}$, $2 \mathrm{H}), 4.43-4.36(\mathrm{~m}, 1 \mathrm{H}), 4.23-4.13(\mathrm{~m}, 1 \mathrm{H}), 3.40(\mathrm{~s}, 2 \mathrm{H}), 3.30(\mathrm{~s}, 2 \mathrm{H})$, 2.21 (s, 2H), 1.88-1.79 (m, 6H), 1.39-1.23 (app br s, 18H); ${ }^{13} \mathrm{C}$ NMR (DMSO-d ${ }_{6}, \delta=39.52$ ppm, $100 \mathrm{MHz}$ ): $\delta$ 171.95, 153.61, 153.04, $149.87,147.41,137.09,136.21,128.05,124.70,124.01,119.90$, 117.92, 113.55, 78.61, 60.45, 46.56, 30.92, 30.12, 27.88, 23.99, 23.39; ${ }^{19}$ F NMR (DMSO-d $6,376 \mathrm{MHz}$ ): $\delta-135.48,-138.91,-139.77$; HRMS (ESI) $m / z$ : anal. calcd for $[\mathrm{M}+\mathrm{Na}]^{+} \mathrm{C}_{32} \mathrm{H}_{40} \mathrm{~F}_{2} \mathrm{~N}_{4} \mathrm{NaO}_{10} \mathrm{~S}$ : 733.2325; found 733.2325.

Di-tert-butyl 2,2'-(((sulfonylbis(oxy))bis(4,1-phenylene))bis-(1Himidazole-5,2-diyl))(2S,2'S)-bis(pyrrolidine-1-carboxylate) (10g).
Yield (32.2 mg, 69\%); ${ }^{1} \mathrm{H}$ NMR (DMSO- $\mathrm{d}_{6}, \delta=2.5 \mathrm{ppm}, 400 \mathrm{MHz}$ ): $\delta 11.99(\mathrm{~s}, 2 \mathrm{H}), 7.88-7.85(\mathrm{~d}, 3 \mathrm{H}), 7.53(\mathrm{~s}, 2 \mathrm{H}), 7.39-7.38(\mathrm{~d}, 3 \mathrm{H})$, 7.30-7.27 (d, 1H), 6.91-6.87 (d, 1H), 4.84-4.77 (d, 2H), 4.03-3.98 (t, $1 \mathrm{H}), 3.53(\mathrm{~s}, 2 \mathrm{H}), 3.29-3.25(\mathrm{~m}, 1 \mathrm{H}), 2.22-2.14(\mathrm{~m}, 2 \mathrm{H}), 2.05-1.93$ (m, 2H), 1.85-1.71 (m, 4H), 1.39-1.15 (app br s, 18H); ${ }^{13} \mathrm{C}$ NMR (DMSO-d ${ }_{6}, \delta=39.52$ ppm, $100 \mathrm{MHz}$ ): $\delta 174.6,153.4,147.9,125.7$, 121.1, 78.3, 59.6, 55.2 , 46.4 , 33.3, 31.0, 30.0, 28.0, 23.9, 23.2; HRMS (ESI) $m / z$ : anal. calcd for $[\mathrm{M}+\mathrm{Na}]^{+} \mathrm{C}_{36} \mathrm{H}_{45} \mathrm{~N}_{6} \mathrm{O}_{8} \mathrm{~S}$ : 721.3014; found 721.3014 .

Di-tert-butyl 2,2'-(((sulfonylbis(oxy))bis(3,1-phenylene))bis-(1Himidazole-5,2-diyl))(2S,2'S)-bis(pyrrolidine-1-carboxylate) (10h). Yield (54.4 mg, 67\%); ${ }^{1} \mathrm{H}$ NMR (DMSO-d ${ }_{6}, \delta=2.5 \mathrm{ppm}, 400 \mathrm{MHz}$ ): ס 12.04-11.97 (br d, 2H), 8.21-7.76 (t, 4H), 7.64-7.61 (d, 1H), 7.52$7.44(\mathrm{q}, 2 \mathrm{H}), 7.37-7.20(\mathrm{~m}, 2 \mathrm{H}), 6.93-6.74(\mathrm{q}, 1 \mathrm{H}), 4.83-4.77(\mathrm{~d}, 2 \mathrm{H})$, $3.53(\mathrm{~s}, 2 \mathrm{H}), 3.39-3.36(\mathrm{~m}, 2 \mathrm{H}), 2.22-2.15(\mathrm{~m}, 2 \mathrm{H}), 1.98-1.79(\mathrm{~m}$, $6 \mathrm{H}), 1.38-1.14$ (app br s, $18 \mathrm{H}) ;{ }^{13} \mathrm{C}$ NMR (DMSO-d ${ }_{6}, \delta=39.52 \mathrm{ppm}$, $100 \mathrm{MHz}$ ): $\delta$ 153.4, 150.4, 138.1, 131.3, 130.5, 123.5, 119.2, 117.8, 116.4, 116.2, 78.2, 55.2, 46.3, 33.3, 27.9, 23.1; HRMS (ESI) $m / z$ : anal. calcd for $[\mathrm{M}+\mathrm{Na}]^{+} \mathrm{C}_{36} \mathrm{H}_{45} \mathrm{~N}_{6} \mathrm{O}_{8} \mathrm{~S}$ : 721.3014; found 721.3014.

tert-Butyl (S)-2-(5-(3-)((4-(2-)((S)-1-(tert-butoxycarbonyl)-pyrrolidin2-yl)-1 $H$-imidazol-5-yl)phenoxy)sulfonyl)oxy)-phenyl)-1H-imidazol-2yl)pyrrolidine-1-carboxylate (10i). Yield (721 mg, 52\%); ${ }^{1} \mathrm{H}$ NMR (DMSO-d ${ }_{6}, \delta=2.5$ ppm, $400 \mathrm{MHz}$ ): $\delta 8.25-8.04$ (m, 6H), 7.66-7.51 (d, $4 \mathrm{H}), 5.12(\mathrm{~s}, 2 \mathrm{H}), 3.63(\mathrm{~s}, 2 \mathrm{H}), 3.41(\mathrm{~s}, 2 \mathrm{H}), 2.38(\mathrm{~s}, 2 \mathrm{H}), 2.07-1.91(\mathrm{~m}$, 6H), 1.38-1.15 (app br s, 18H); ${ }^{13} \mathrm{C}$ NMR (DMSO-d $\mathrm{d}_{6}, v=39.52 \mathrm{ppm}$, $100 \mathrm{MHz}$ ): $\delta$ 153.71, 152.45, 150.67, 150.56, 150.22, 149.56, 131.39, 127.37, 124.78, 122.07, 117.89, 116.19, 115.71, 79.19, 52.97, 46.45, 32.89, 31.98, 27.70, 23.92, 23.38; HRMS (ESI) $\mathrm{m} / \mathrm{z}$ : anal. calcd for [M + $\mathrm{H}]^{+} \mathrm{C}_{36} \mathrm{H}_{45} \mathrm{~N}_{6} \mathrm{O}_{8} \mathrm{~S}:$ 721.3014; found 721.3016.

Di-tert-butyl 2,2'-(((sulfonylbis(oxy))bis(4-fluoro-3,1-phenylene))bis(1H-imidazole-5,2-diyl))(2S,2'S)-bis(pyrrolidine-1carboxylate) (10j). Yield (305 mg, 63\%); ${ }^{1} \mathrm{H}$ NMR (DMSO- $\mathrm{d}_{6}, v$ $=2.5 \mathrm{ppm}, 400 \mathrm{MHz}): \delta 12.01(\mathrm{~s}, 2 \mathrm{H}), 7.92-7.91(\mathrm{~d}, 2 \mathrm{H}), 7.82$ $(\mathrm{s}, 2 \mathrm{H}), 7.58(\mathrm{~s}, 2 \mathrm{H}), 7.52-7.47(\mathrm{t}, 2 \mathrm{H}), 4.82-4.76(\mathrm{~d}, 2 \mathrm{H}), 3.52$ $(\mathrm{s}, 2 \mathrm{H}), 3.37-3.17(\mathrm{~m}, 2 \mathrm{H}), 2.22-2.13(\mathrm{~m}, 2 \mathrm{H}), 1.95-1.84(\mathrm{t}$, $2 \mathrm{H}$ ), 1.37-1.14 (app br s, $18 \mathrm{H}$ ); ${ }^{13} \mathrm{C}$ NMR $\left(\right.$ DMSO-d $_{6}, \delta=$ $39.52 \mathrm{ppm}, 100 \mathrm{MHz}): \delta 153.35,152.22,150.24,136.98$, $136.87,133.21,125.00,118.39,117.68,113.11$, 78.24, 55.22, 46.29, 33.23, 31.78, 27.83, 23.77, 23.07; ${ }^{19}$ F NMR (DMSO-d 6 , $376 \mathrm{MHz}$ ): $\delta-133.22$; HRMS (ESI) $m / z$ : anal. calcd for $[\mathrm{M}+\mathrm{H}]^{+}$ $\mathrm{C}_{36} \mathrm{H}_{43} \mathrm{~F}_{2} \mathrm{~N}_{6} \mathrm{O}_{8} \mathrm{~S}$ : 757.2826; found 757.2828.

\section{General procedure for synthesis of inhibitors}

A mixture of precursor $(0.151 \mathrm{mmol})$ in $\mathrm{CF}_{3} \mathrm{CO}_{2} \mathrm{H}(3 \mathrm{~mL}) / \mathrm{CH}_{2} \mathrm{Cl}_{2}$ $(3 \mathrm{~mL})$ was stirred at room temperature for $30 \mathrm{~m}$. The volatile component was removed in vacuo, 1-ethyl-3-(3dimethylaminopropyl)carbodiimide $(0.392 \mathrm{mmol})$, hydroxybenzotriazole hydrate $(0.392 \mathrm{mmol})$, capping group $(0.362$ mmol) were added in batches over 4 min to a solution of $\mathrm{N}, \mathrm{N}$ diisopropylethylamine $(0.754 \mathrm{mmol})$ in $\mathrm{CH}_{2} \mathrm{Cl}_{2}(10 \mathrm{~mL})$ and the reaction mixture stirred at room temperature for overnight. The mixture was then poured in $1 \mathrm{~N}$ aq. $\mathrm{HCl}$ solution and extracted with $\mathrm{CH}_{2} \mathrm{Cl}_{2}$. The organic layer dried over magnesium sulfate, and then was purified by the column chromatography $\left(\mathrm{CH}_{2} \mathrm{Cl}_{2} /\right.$ $\mathrm{MeOH})$. The residue was obtained as solid. 
3-((S)-1-((R)-2-((Methoxycarbonyl)amino)-2-phenylacetyl)pyrrolidine-2-carboxamido)phenyl (4- $((S)-1-((R)-2-(($ methoxycarbonyl)amino)-2-phenylacetyl)pyrrolidine-2-carboxamido)phenyl) sulfate (11). Yield (106.7 mg, 83\%); ${ }^{1} \mathrm{H}$ NMR (DMSO$\left.\mathrm{d}_{6}, \delta=2.5 \mathrm{ppm}, 400 \mathrm{MHz}\right): \delta 10.63-10.31 / 10.27 / 10.17(\mathrm{~m} / \mathrm{s} / \mathrm{s}$, $0.87 \mathrm{H}+0.5 \mathrm{H}+0.57 \mathrm{H}), 7.91-7.89(\mathrm{~d}, 1 \mathrm{H}), 7.80-7.72(\mathrm{~m}, 3 \mathrm{H})$, 7.68-7.56 (m, 2H), 7.50-7.26 (m, 12H), 7.17-7.05 (m, 2H), $5.53-5.42 / 5.37-5.34 / 4.94-4.90(\mathrm{~m} / \mathrm{t} / \mathrm{t}, 1.61 \mathrm{H}+0.19 \mathrm{H}+0.18 \mathrm{H})$, 4.61-4.39 (m, 2H), 3.86-3.81 (qui, 1H), 3.73-3.66 (m, 1H), $3.54(\mathrm{~s}, 6 \mathrm{H}), 3.22-3.17(\mathrm{q}, 1 \mathrm{H}), 3.13-3.09(\mathrm{t}, 1 \mathrm{H}), 2.32-2.14(\mathrm{~m}$, $1 \mathrm{H}), 2.07-1.76(\mathrm{~m}, 8 \mathrm{H}), 1.23 / 0.85(2 \mathrm{~s}, 0.30 \mathrm{H}+0.10 \mathrm{H}) ;{ }^{13} \mathrm{C}$ NMR (DMSO-d $\left.\mathrm{d}_{6}, \delta=39.52 \mathrm{ppm}, 100 \mathrm{MHz}\right): \delta 170.59,168.40$, 156.09, 149.89, 145.03, 140.74, 138.70, 137.21, 130.65, 128.62, 128.06, 121.61, 120.65, 118.31, 115.50, 111.35, 60.71, 56.71, $51.65,46.98,29.25,24.30$; HRMS (ESI) $m / z$ : anal. calcd for [M $+\mathrm{Na}]^{+} \mathrm{C}_{42} \mathrm{H}_{44} \mathrm{~N}_{6} \mathrm{NaO}_{12} \mathrm{~S}$ : 879.2630; found 879.2631.

3-((S)-1-((Methoxycarbonyl)-D-valyl)pyrrolidine-2-carboxamido) phenyl (4-((S)-1-((methoxycarbonyl)-D-valyl)-pyrrolidine-2carboxamido)phenyl) sulfate (12). Yield $(46.7 \mathrm{mg}, 71 \%) ;{ }^{1} \mathrm{H}$ NMR (DMSO-d $\mathrm{d}_{6}, \delta=2.5 \mathrm{ppm}, 400 \mathrm{MHz}$ ): $\delta 10.64 / 10.54 / 10.13 / 10.02$ (4 s, $0.2 \mathrm{H}+0.2 \mathrm{H}+0.86 \mathrm{H}+0.82 \mathrm{H}), 7.87(\mathrm{~s}, 1 \mathrm{H}), 7.76-7.73(\mathrm{~d}, 2 \mathrm{H}), 7.61-$ 7.59 (d, 2H), 7.51-7.36 (m, 5H), 7.14-7.08 (m, 1H), 4.43-4.39 (q, $2 \mathrm{H}), 4.12-4.07(\mathrm{t}, 2 \mathrm{H}), 3.79(\mathrm{~s}, 2 \mathrm{H}), 3.64-3.58(\mathrm{q}, 2 \mathrm{H}), 3.55-3.53$ (app s, 6H), 3.48-3.45 (m, 1H), 2.16-2.08 (m, 2H), 2.04-1.92 (m, 8H), 0.89-0.86 (t, 10H), 0.80-0.77 (t, 1H), 0.72-0.68 (m, 1H); ${ }^{13} \mathrm{C}$ NMR (DMSO-d 6 , $v=39.52 \mathrm{ppm}, 100 \mathrm{MHz}$ ): $\delta 171.01,170.71$, 170.24, 156.92, 149.85, 144.94, 140.71, 138.67, 130.61, 121.57, 120.50, 118.19, 115.42, 111.22, 60.32, 60.27, 58.01, 51.56, 47.11, 29.76, 29.41, 29.37, 24.39, 24.35, 19.09, 18.32; HRMS (ESI) $\mathrm{m} / \mathrm{z}$ : anal. calcd for $[\mathrm{M}+\mathrm{Na}]^{+} \mathrm{C}_{36} \mathrm{H}_{48} \mathrm{~N}_{6} \mathrm{NaO}_{12} \mathrm{~S}$ : 811.2943; found 811.2913.

Bis(2-fluoro-3-((S)-1-((R)-2-((methoxycarbonyl)amino)-2phenylacetyl)pyrrolidine-2-carboxamido)phenyl) sulfate (13). Yield $(41.0 \mathrm{mg}, 49 \%) ;{ }^{1} \mathrm{H}$ NMR (DMSO- ${ }_{6}, v=2.5 \mathrm{ppm}, 400$ MHz): $\delta 10.21(\mathrm{~s}, 1 \mathrm{H}), 10.10(\mathrm{~s}, 1 \mathrm{H}), 8.02-7.97(\mathrm{q}, 2 \mathrm{H}), 7.72-$ $7.66(\mathrm{q}, 2 \mathrm{H}), 7.41-7.40(\mathrm{~m}, 6 \mathrm{H}), 7.37-7.29(\mathrm{~m}, 7 \mathrm{H}), 7.27-7.25 /$ $7.12-7.10(2 \mathrm{~d}, 0.48 \mathrm{H}+0.35 \mathrm{H}), 5.51-5.45(\mathrm{q}, 2 \mathrm{H}), 4.69-4.67$ $(\mathrm{m}, 1 \mathrm{H}), 4.57-4.55(\mathrm{~m}, 1 \mathrm{H}), 3.81(\mathrm{~s}, 1 \mathrm{H}), 3.71-3.65(\mathrm{~m}, 1 \mathrm{H})$, $3.53(\mathrm{~s}, 6 \mathrm{H}), 3.20-3.14(\mathrm{q}, 1 \mathrm{H}), 3.10-3.08(\mathrm{~d}, 1 \mathrm{H}), 2.18-2.16(\mathrm{~d}$, $1 \mathrm{H}), 2.03-1.78(\mathrm{~m}, 7 \mathrm{H}), 1.23 / 0.85(2 \mathrm{~s}, 2.02 \mathrm{H}+0.62 \mathrm{H}) ;{ }^{13} \mathrm{C}$ NMR (DMSO-d ${ }_{6}, \delta=39.52 \mathrm{ppm}, 126 \mathrm{MHz}$ ): $\delta 170.92,168.45$, 168.25, 156.33, 155.97, 136.74, 128.60, 128.38, 128.35, 127.99, 124.76, 123.15, 118.26, 118.03, 60.26, 56.65, 51.55, 46.85, 29.14, 24.69, 24.27; ${ }^{19} \mathrm{~F}$ NMR (DMSO-d 6 , $376 \mathrm{MHz}$ ): $\delta-74.67$, $-139.84,-139.84$; HRMS (ESI) $m / z$ : anal. calcd for $[\mathrm{M}+\mathrm{Na}]^{+}$ $\mathrm{C}_{42} \mathrm{H}_{42} \mathrm{~F}_{2} \mathrm{~N}_{6} \mathrm{NaO}_{12} \mathrm{~S}$ : 915.2442; found 915.2445.

Bis(2-fluoro-5-((S)-1-((R)-2-((methoxycarbonyl)amino)-2phenylacetyl)pyrrolidine-2-carboxamido)phenyl) sulfate (14). Yield $(63.4 \mathrm{mg}, 52 \%) ;{ }^{1} \mathrm{H}$ NMR (DMSO- ${ }_{6}, \delta=2.5 \mathrm{ppm}, 400$ $\mathrm{MHz}): \delta 10.64 / 10.47 / 10.32 / 10.28(4 \mathrm{~s}, 0.06 \mathrm{H}+0.3 \mathrm{H}+0.33 \mathrm{H}+$ 1.19H), 8.13-8.01 (m, 2H), 7.87-7.72 (m, 2H), $7.65(\mathrm{~s}, 2 \mathrm{H})$, 7.55-7.50 (t, 2H), 7.42-7.29 (m, 8H), 7.25-7.23/7.06-7.03 (2 $\mathrm{m}, 0.52 \mathrm{H}+0.74 \mathrm{H}) 7.06-7.04(\mathrm{t}, 1 \mathrm{H}), 5.52-5.47 / 5.35-5.33 /$ $4.87-4.85(\mathrm{~m} / \mathrm{d} / \mathrm{d}, 1.56 \mathrm{H}+0.26 \mathrm{H}+0.20 \mathrm{H}), 4.48-4.35(\mathrm{~m}, 2 \mathrm{H})$, $3.84-3.81(\mathrm{~m}, 2 \mathrm{H}), 3.53(\mathrm{~s}, 6 \mathrm{H}), 3.20-3.09(\mathrm{~m}, 2 \mathrm{H}), 2.04-1.77$ $(\mathrm{m}, 8 \mathrm{H}), 1.23 / 0.85(2 \mathrm{~s}, 0.35 \mathrm{H}+0.07 \mathrm{H}) ;{ }^{13} \mathrm{C}$ NMR $\left(\right.$ DMSO- $_{6}$, $\delta=39.52 \mathrm{ppm}, 100 \mathrm{MHz}): \delta 170.71,168.39,156.07,149.70$, 147.73, 137.20, 136.26, 128.59, 128.03, 119.96, 117.92, 113.47, $60.69,56.67,51.63,46.96,29.21,24.31 ;{ }^{19}$ F NMR (DMSO-d $\mathrm{d}_{6}$, $376 \mathrm{MHz}$ ): $\delta-135.20$; HRMS (ESI) $\mathrm{m} / \mathrm{z}$ : anal. calcd for [M + $\mathrm{Na}]^{+} \mathrm{C}_{42} \mathrm{H}_{42} \mathrm{~F}_{2} \mathrm{~N}_{6} \mathrm{NaO}_{12} \mathrm{~S}$ : 915.2442; found 915.2443.

2-Fluoro-3-((S)-1-((R)-2-((methoxycarbonyl)amino)-2-phenylacetyl)pyrrolidine-2-carboxamido)phenyl (2-fluoro-5-((S)1-((R)-2-((methoxycarbonyl)amino)-2-phenylacetyl)-pyrrolidine-2-carboxamido)phenyl) sulfate (15). Yield (112 mg, 58\%); ${ }^{1} \mathrm{H}$ NMR (DMSO-d $\mathrm{d}_{6}, \delta=2.5 \mathrm{ppm}, 400 \mathrm{MHz}$ ): $\delta$ 10.46/10.27/ $10.21 / 10.11(4 \mathrm{~s}, 0.57 \mathrm{H}+0.53 \mathrm{H}+0.32 \mathrm{H}+0.36 \mathrm{H}), 8.11-7.80$ $(\mathrm{m}, 2 \mathrm{H}), 7.74-7.64(\mathrm{~m}, 3 \mathrm{H}), 7.55-7.50(\mathrm{t}, 1 \mathrm{H}), 7.39-7.21(\mathrm{~m}$, $11 \mathrm{H}), 7.09-7.04(\mathrm{~d}, 2 \mathrm{H}), 5.51-5.20 / 5.10-5.08 / 4.86-4.84(\mathrm{~m} / \mathrm{d} /$ $\mathrm{d}, 1.96 \mathrm{H}+0.06 \mathrm{H}+0.08 \mathrm{H}), 4.68 / 4.57-4.55 / 4.46-4.43 / 4.36-$ $4.34(4 \mathrm{~m}, 0.28 \mathrm{H}+0.40 \mathrm{H}+0.46 \mathrm{H}+0.62 \mathrm{H}), 3.81-3.67(\mathrm{~m}, 2 \mathrm{H})$, $3.52(\mathrm{~s}, 6 \mathrm{H}), 3.17-3.08(\mathrm{~m}, 2 \mathrm{H}), 2.27-2.16(\mathrm{~m}, 1 \mathrm{H}), 1.99-1.80$ $(\mathrm{m}, 7 \mathrm{H}), 1.23 / 0.85(2 \mathrm{~s}, 0.31 \mathrm{H}+0.10 \mathrm{H}) ;{ }^{13} \mathrm{C}$ NMR $\left(\right.$ DMSO-d $_{6}$, $\delta=39.52 \mathrm{ppm}, 100 \mathrm{MHz}): \delta 170.92,168.38,156.30,156.05$, $149.85,147.40,137.16,136.77,136.25,136.08,128.58,128.35$, 128.01, 124.81, 123.48, 123.06, 119.72, 117.96, 117.78, 113.31, $60.67,56.65,51.54,46.94,29.18,24.30 ;{ }^{19} \mathrm{~F}$ NMR (DMSO-d 6 , $376 \mathrm{MHz}$ ): $\delta-73.82,-135.52,-139.50,-139.97$; HRMS (ESI) $m / z$ : anal. calcd for $[\mathrm{M}+\mathrm{Na}]^{+} \mathrm{C}_{42} \mathrm{H}_{42} \mathrm{~F}_{2} \mathrm{~N}_{6} \mathrm{NaO}_{12} \mathrm{~S}$ : 915.2442; found 915.2441 .

Bis(2-fluoro-5-((S)-1-((methoxycarbonyl)-D-valyl)pyrrolidine2-carboxamido)phenyl) sulfate (16). Yield $(77.7 \mathrm{mg}, 80 \%) ;{ }^{1} \mathrm{H}$ NMR (DMSO-d $\left.\mathrm{d}_{6}, \delta=2.5 \mathrm{ppm}, 500 \mathrm{MHz}\right): \delta 10.63 / 10.15(2 \mathrm{~s}$, $0.35 \mathrm{H}+1.62 \mathrm{H}), 8.04(\mathrm{~s}, 2 \mathrm{H}), 7.63-7.61(\mathrm{~d}, 2 \mathrm{H}), 7.52-7.48(\mathrm{t}, 2 \mathrm{H})$, 7.36-7.35 (d, 2H), 4.38-4.36 (d, 2H), 4.11-4.07 (t, 2H), 3.78$3.74(\mathrm{~m}, 2 \mathrm{H}), 3.63-3.57(\mathrm{~m}, 2 \mathrm{H}), 3.53(\mathrm{~s}, 6 \mathrm{H}), 2.15-2.08(\mathrm{~m}, 2 \mathrm{H})$, 2.08-1.84 (m, 8H), 0.88-0.86 (t, 10H), 0.77-0.76 (d, 1H), 0.67$0.67(\mathrm{~d}, 1 \mathrm{H}) ;{ }^{13} \mathrm{C}$ NMR (DMSO-d ${ }_{6}, \delta=39.52 \mathrm{ppm}, 126 \mathrm{MHz}$ ): $\delta 170.87,170.22,156.93,149.64,147.66,136.25,120.42$, $119.35,117.33,113.56,113.09,60.63,59.92,57.96,51.42$, 29.95, 24.44, 19.09, 18.29; ${ }^{19} \mathrm{~F}$ NMR (DMSO- ${ }_{6}, 376 \mathrm{MHz}$ ): $\delta-135.35$; HRMS (ESI) $m / z$ : anal. calcd for $[\mathrm{M}+\mathrm{Na}]^{+} \mathrm{C}_{36} \mathrm{H}_{46}{ }^{-}$ $\mathrm{F}_{2} \mathrm{~N}_{6} \mathrm{NaO}_{12} \mathrm{~S}: 847.2755$; found 847.2752.

2-Fluoro-3-((S)-1-((methoxycarbonyl)-D-valyl)pyrrolidine-2carboxamido)phenyl (2-fluoro-5-((S)-1-((methoxycarbonyl)-Dvalyl)pyrrolidine-2-carboxamido)phenyl) sulfate (17). Yield (40.3 mg, 39\%); ${ }^{1} \mathrm{H}$ NMR (DMSO-d $\mathrm{d}_{6}, \delta=2.5 \mathrm{ppm}, 400 \mathrm{MHz}$ ): $\delta 10.64 / 10.45 / 10.16 / 9.97(4 \mathrm{~s}, 0.16 \mathrm{H}+0.22 \mathrm{H}+0.81 \mathrm{H}+0.71 \mathrm{H})$, 8.08-8.07 (d, 1H), 7.99-7.82 (m, 1H), 7.64-7.60 (m, 1H), 7.53$7.48(\mathrm{t}, 1 \mathrm{H}), 7.44-7.28(\mathrm{~m}, 4 \mathrm{H}), 5.17-5.15 / 5.03-5.01 / 4.60-4.58$ $(3 \mathrm{~d}, 0.14 \mathrm{H}+0.16 \mathrm{H}+0.77 \mathrm{H}), 4.38-4.37(\mathrm{~d}, 1 \mathrm{H}), 4.12-4.07(\mathrm{t}$, $2 \mathrm{H}), 3.87-3.71(\mathrm{~m}, 2 \mathrm{H}), 3.64-3.58(\mathrm{~m}, 1 \mathrm{H}), 3.53-3.50$ (app s, $6 \mathrm{H}), 3.46-3.45(\mathrm{~m}, 1 \mathrm{H}) 2.21-2.09(\mathrm{~m}, 2 \mathrm{H}), 1.99-1.81(\mathrm{~m}, 8 \mathrm{H})$, $1.23(\mathrm{~s}, 0.32 \mathrm{H}), 0.89-0.66(\mathrm{~m}, 12 \mathrm{H}) ;{ }^{13} \mathrm{C}$ NMR $\left(\right.$ DMSO- $_{6}, \delta=$ $39.52 \mathrm{ppm}, 100 \mathrm{MHz}$ ): $\delta 170.90,170.22,156.84,149.90$, 147.44, 146.40, 143.89, 137.12, 136.06, 128.18, 124.71, 123.31, 119.81, 117.89, 113.39, 60.28, 58.00, 51.54, 47.09, 29.75, $24.38,19.07,18.30 ;{ }^{19} \mathrm{~F}$ NMR (DMSO- ${ }_{6}, 376 \mathrm{MHz}$ ): $\delta-134.98$, $-135.41,-138.11,-139.92$; HRMS (ESI) $m / z$ : anal. calcd for $[\mathrm{M}+\mathrm{Na}]^{+} \mathrm{C}_{36} \mathrm{H}_{46} \mathrm{~F}_{2} \mathrm{~N}_{6} \mathrm{NaO}_{12} \mathrm{~S}$ : 847.2755; found 847.2754.

Bis(2-fluoro-3-((S)-1-((methoxycarbonyl)-D-valyl)pyrrolidine-2carboxamido)phenyl) sulfate (18). Yield $(97.9 \mathrm{mg}, 74 \%) ;{ }^{1} \mathrm{H}$ NMR (DMSO- $\left.\mathrm{d}_{6}, v=2.5 \mathrm{ppm}, 400 \mathrm{MHz}\right): \delta 10.45 / 10.14 / 9.97(3 \mathrm{~s}, 0.33 \mathrm{H}$ 
$+0.20 \mathrm{H}+1.44 \mathrm{H}), 7.98-7.81(\mathrm{~m}, 2 \mathrm{H}), 7.48-7.29(\mathrm{~m}, 6 \mathrm{H}), 5.17-5.15 /$ $4.60-4.58(2 \mathrm{~d}, 0.27 \mathrm{H}+1.48 \mathrm{H}), 4.12-4.07(\mathrm{t}, 1 \mathrm{H}), 3.87-3.79(\mathrm{~m}$, $2 \mathrm{H}), 3.66-3.60$ (m, 4H), 3.50 (s, 6H), 2.25-2.11 (m, 2H), 1.98-1.85 (m, 8H), $1.23(\mathrm{~s}, 0.15 \mathrm{H}), 0.89-0.71(\mathrm{~m}, 12 \mathrm{H}) ;{ }^{13} \mathrm{C}$ NMR (DMSO-d ${ }_{6}$, $\delta=39.52 \mathrm{ppm}, 100 \mathrm{MHz}): \delta$ 170.53, 156.87, 146.52, 144.01, 137.14, 128.15, 124.70, 123.42, 118.19, 59.91, 58.04, 51.45, 48.64, 47.13, 29.75, 29.16, 24.37, 19.08, 18.32; ${ }^{19}$ F NMR (DMSO-d 6,376 MHz): $\delta-78.40,-142.54,-144.44$; HRMS (ESI) $m / z$ : anal. calcd for $[\mathrm{M}+\mathrm{Na}]^{+} \mathrm{C}_{36} \mathrm{H}_{46} \mathrm{~F}_{2} \mathrm{~N}_{6} \mathrm{NaO}_{12} \mathrm{~S}$ : 847.2755; found 847.2757.

Bis(2-fluoro-5-(2-((S)-1-((methoxycarbonyl)-D-valyl)pyrrolidin2-yl)-1H-imidazol-5-yl)phenyl) sulfate (19). Yield (34.5 mg, 52\%); ${ }^{1} \mathrm{H}$ NMR (DMSO-d ${ }_{6}, \delta=2.5 \mathrm{ppm}, 400 \mathrm{MHz}$ ): $\delta 8.08-8.06(\mathrm{~d}, 2 \mathrm{H})$, $8.01(\mathrm{~s}, 2 \mathrm{H}), 7.92-7.91(\mathrm{~d}, 2 \mathrm{H}), 7.80(\mathrm{~s}, 1 \mathrm{H}), 7.73-7.68(\mathrm{t}, 1 \mathrm{H}), 7.59-$ $7.46(\mathrm{~m}, 1 \mathrm{H}), 7.25-7.23(\mathrm{~d}, 1 \mathrm{H}), 5.69-5.67 / 5.14-5.12(2 \mathrm{~d}, 0.35 \mathrm{H}+$ $1.32 \mathrm{H}), 4.17-4.13(\mathrm{t}, 2 \mathrm{H}), 3.87-3.86(\mathrm{~d}, 2 \mathrm{H}), 3.68-3.62(\mathrm{q}, 2 \mathrm{H}), 3.53$ (s, 6H), 2.32 (s, 2H), 2.08-1.67 (m, 8H), 1.23 (s, 0.47H), 0.90-0.29 $(\mathrm{m}, 12 \mathrm{H}) ;{ }^{13} \mathrm{C}$ NMR (DMSO-d $6, \delta=39.52 \mathrm{ppm}, 100 \mathrm{MHz}$ ): $\delta$ 170.71, 156.88, 149.66, 137.07, 136.94, 126.54, 120.06, 118.57, $115.78,57.87,53.48,51.58,46.95,30.88,29.69,24.28,19.25$, 18.57, 18.41, 17.93; ${ }^{19} \mathrm{~F}$ NMR (DMSO-d $6,376 \mathrm{MHz}$ ): $\delta-74.27$; HRMS (ESI) $m / z$ : anal. calcd for $[\mathrm{M}+\mathrm{H}]^{+} \mathrm{C}_{40} \mathrm{H}_{49} \mathrm{~F}_{2} \mathrm{~N}_{8} \mathrm{O}_{10} \mathrm{~S}$ : 871.3255; found 871.3256.

Bis(2-fluoro-5-(2-((S)-1-((methoxycarbonyl)-L-valyl)pyrrolidin2-yl)-1H-imidazol-5-yl)phenyl) sulfate (20). Yield (90.1 mg, 48\%); ${ }^{1} \mathrm{H}$ NMR (DMSO-d $\left.\mathrm{d}_{6}, \delta=2.5 \mathrm{ppm}, 400 \mathrm{MHz}\right): \delta 8.06-8.05(\mathrm{~d}, 2 \mathrm{H})$, 7.99 (s, 2H), 7.90-7.88 (d, 2H), 7.71-7.67 (t, 2H), 7.30-7.28 (d, 2H), $5.45 / 5.11-5.08(\mathrm{~m} / \mathrm{t}, 0.13 \mathrm{H}+1.99 \mathrm{H}), 4.10-4.06(\mathrm{t}, 2 \mathrm{H}), 3.86-3.76$ $(\mathrm{m}, 4 \mathrm{H}), 3.53(\mathrm{~s}, 6 \mathrm{H}), 2.32-2.28(\mathrm{t}, 2 \mathrm{H}), 2.14-2.10(\mathrm{~m}, 2 \mathrm{H}), 2.07-$ $1.94(\mathrm{~m}, 6 \mathrm{H}), 1.23$ (s, 0.46H), 0.81-0.75 (q, 12H); ${ }^{13} \mathrm{C}$ NMR (DMSO$\left.\mathrm{d}_{6}, \delta=39.52 \mathrm{ppm}, 100 \mathrm{MHz}\right): \delta$ 170.95, 158.36, 158.03, 156.88, $149.87,137.07,136.93,126.37,119.84,118.72$, 118.55, 57.93, $53.34,51.52,47.05,31.00,29.30,24.58,19.13,17.99 ;{ }^{19} \mathrm{~F}$ NMR (DMSO-d 6 , $376 \mathrm{MHz}$ ): $\delta-78.32,-138.05$; HRMS (ESI) $\mathrm{m} / z$ : anal. calcd for $[\mathrm{M}+\mathrm{H}]^{+} \mathrm{C}_{40} \mathrm{H}_{49} \mathrm{~F}_{2} \mathrm{~N}_{8} \mathrm{O}_{10} \mathrm{~S}$ : 871.3255; found 871.3254.

Bis(2-fluoro-5-(2-((S)-1-((R)-2-((methoxycarbonyl)amino)-2phenylacetyl)pyrrolidin-2-yl)-1H-imidazol-5-yl)phenyl) sulfate (21). Yield (34.5 mg, 73\%); ${ }^{1} \mathrm{H}$ NMR (DMSO- $\mathrm{d}_{6}, \delta=2.5 \mathrm{ppm}$, $400 \mathrm{MHz}): \delta 8.11-8.03(\mathrm{~m}, 4 \mathrm{H}), 7.98-7.93(\mathrm{~m}, 2 \mathrm{H}), 7.79-7.71$ $(\mathrm{m}, 2 \mathrm{H}), 7.68-7.64(\mathrm{t}, 2 \mathrm{H}), 7.39-7.34(\mathrm{~m}, 6 \mathrm{H}), 7.29(\mathrm{~s}, 2 \mathrm{H}), 6.99$ $(\mathrm{s}, 2 \mathrm{H}), 5.65-5.63 / 5.50-5.49 / 5.40-5.38(3 \mathrm{~d}, 0.51 \mathrm{H}+1.37 \mathrm{H}+$ $0.49 \mathrm{H}), 5.17-5.16(\mathrm{~d}, 2 \mathrm{H}), 3.93-3.82(\mathrm{~m}, 2 \mathrm{H}), 3.53-3.51$ (app s, $6 \mathrm{H}), 3.18-3.12(\mathrm{q}, 2 \mathrm{H}), 2.22-2.15(\mathrm{~m}, 2 \mathrm{H}), 2.02-2.00(\mathrm{~d}, 4 \mathrm{H})$, $1.87(\mathrm{~s}, 2 \mathrm{H}), 1.23(\mathrm{~s}, 0.72 \mathrm{H}) ;{ }^{13} \mathrm{C}$ NMR (DMSO-d $6, \delta=$ $39.52 \mathrm{ppm}, 100 \mathrm{MHz}): \delta 168.88,155.95,151.56,149.53$, 137.11, 136.95, 128.67, 128.27, 128.11, 127.78, 126.70, 120.27, 118.82, 118.63, 115.87, 56.99, 53.80, 51.61, 46.94, 30.91, 24.10; ${ }^{19} \mathrm{~F}$ NMR (DMSO-d 6 , $376 \mathrm{MHz}$ ): $\delta-78.97,-133.12$; HRMS (ESI) $m / z$ : anal. calcd for $[\mathrm{M}+\mathrm{H}]^{+} \mathrm{C}_{46} \mathrm{H}_{45} \mathrm{~F}_{2} \mathrm{~N}_{8} \mathrm{O}_{10} \mathrm{~S}$ : 939.2942; found 939.2939.

3-(2-((S)-1-((R)-2-((Methoxycarbonyl)amino)-2-phenyl-acetyl) pyrrolidin-2-yl)-1H-imidazol-5-yl)phenyl (4-(2- $((S)-1-((R)-2-$ ((methoxycarbonyl)amino)-2-phenylacetyl)pyrrolidin-2-yl)-1 Himidazol-5-yl)phenyl) sulfate (22). Yield (53.3 mg, 35\%); ${ }^{1} \mathrm{H}$ NMR (DMSO-d 6 , $\delta=2.5$ ppm, $400 \mathrm{MHz}): \delta 8.19-8.06(\mathrm{~m}, 2 \mathrm{H})$, 7.98-7.96 (d, 2H), 7.91-7.83 (m, 2H), 7.73-7.64 (m, 5H), 7.39$7.25(\mathrm{~m}, 10 \mathrm{H}), 7.03(\mathrm{~s}, 1 \mathrm{H}), 5.53-5.49 / 5.45-5.43 / 5.40-5.38(\mathrm{t} / \mathrm{d} / \mathrm{d}$, $1.51 \mathrm{H}+0.25 \mathrm{H}+0.18 \mathrm{H}), 5.18(\mathrm{~m}, 2 \mathrm{H}), 3.91-3.85(\mathrm{~d}, 1 \mathrm{H}), 3.80-$
3.71 (m, 1H), 3.54-3.51 (app br s, 6H), 3.46-3.38 (d, 1H), 3.17$3.15(\mathrm{~d}, 1 \mathrm{H}), 2.37-2.22(\mathrm{~m}, 2 \mathrm{H}), 2.08-1.89(\mathrm{~m}, 6 \mathrm{H}), 1.23(\mathrm{~s}$, $0.20 \mathrm{H}$ ); ${ }^{13} \mathrm{C}$ NMR (DMSO-d $\left.{ }_{6}, \delta=39.52 \mathrm{ppm}, 126 \mathrm{MHz}\right): \delta 168.99$, 156.34 , 155.97, 150.33, 149.40, 136.86, 131.50, 128.69, 128.62, $128.31,128.12,127.79,127.44,127.23,124.83,122.11,122.06$, 117.94, 115.90, 57.00, 56.73, 53.48, 51.62, 47.01, 30.88, 24.14; HRMS (ESI) $m / z$ : anal. calcd for $[\mathrm{M}+\mathrm{H}]^{+} \mathrm{C}_{46} \mathrm{H}_{47} \mathrm{~N}_{8} \mathrm{O}_{10} \mathrm{~S}$ : 903.3130; found 903.3130.

3-(2-((S)-1-((Methoxycarbonyl)-D-valyl)pyrrolidin-2-yl)-1Himidazol-5-yl)phenyl (4-(2-((S)-1-((methoxycarbonyl)-D-valyl)pyrrolidin-2-yl)-1H-imidazol-5-yl)phenyl) sulfate (23). Yield (107 mg, 86\%); ${ }^{1} \mathrm{H}$ NMR (DMSO-d 6 , $\delta=2.5 \mathrm{ppm}, 400 \mathrm{MHz}$ ): $\delta$ 8.13-8.08 (d, 2H), 7.95-7.87 (m, 4H), 7.78-7.63 (m, 3H), 7.54-7.48 (m, 1H), 7.30-7.23 (m, 2H), 5.80-5.74/5.19-5.10 (d $\left.\mathrm{m}^{-1}, 0.25 \mathrm{H}+1.73 \mathrm{H}\right), 4.18-4.07(\mathrm{~m}, 2 \mathrm{H}), 3.88-3.78(\mathrm{~m}, 2 \mathrm{H})$, 3.68-3.66 (d, 2H), 3.53 (s, 6H), 2.36 (s, 2H), 2.08-1.96 (m, 8H), $1.23(\mathrm{~s}, 0.39 \mathrm{H}), 0.90-0.68(\mathrm{~m}, 12 \mathrm{H}), 0.33(\mathrm{~s}, 1 \mathrm{H}) ;{ }^{13} \mathrm{C} \mathrm{NMR}$ $\left(\right.$ DMSO$\left.^{-d_{6}}, \delta=39.52 \mathrm{ppm}, 100 \mathrm{MHz}\right): \delta 170.80,158.49$, 158.15, 156.87, 150.32, 149.57, 131.43, 127.33, 124.70, 122.08, $120.79,117.78,116.21,115.85,57.84,53.19,51.59,47.00$, 30.89, 29.70, 24.34, 19.28, 17.89; HRMS (ESI) $\mathrm{m} / \mathrm{z}$ : anal. calcd for $[\mathrm{M}+\mathrm{H}]^{+} \mathrm{C}_{40} \mathrm{H}_{51} \mathrm{~N}_{8} \mathrm{O}_{10} \mathrm{~S}$ : 835.3443; found 835.3446.

3-(2-((S)-1-((Methoxycarbonyl)-L-valyl)pyrrolidin-2-yl)-1Himidazol-5-yl)phenyl (4-(2- $((S)-1-(($ methoxycarbonyl)-L-valyl)pyrrolidin-2-yl)-1H-imidazol-5-yl)phenyl) sulfate (24). Yield (110 mg, 54\%); ${ }^{1} \mathrm{H}$ NMR (DMSO-d $6, \delta=2.5$ ppm, $400 \mathrm{MHz}$ ): $\delta$ 12.37-12.39/12.16-12.13/11.92-11.86 (3 d, 0.14H+0.18H+1.51H), 7.85-7.83 (d, 2H), 7.76-7.73 (m, 2H), $7.61(\mathrm{~s}, 1 \mathrm{H}), 7.54(\mathrm{~s}, 1 \mathrm{H}), 7.48-$ $7.44(\mathrm{t}, 1 \mathrm{H}), 7.39-7.36$ (d, 2H), 7.31-7.26 (t, 2H), 7.19-7.16 (q, 1H), $5.22 / 5.08-5.03(2 \mathrm{~m}, 0.14 \mathrm{H}+1.86 \mathrm{H}), 4.11-4.00(\mathrm{~m}, 2 \mathrm{H}), 3.78(\mathrm{~s}, 2 \mathrm{H})$, $3.53(\mathrm{~s}, 6 \mathrm{H}), 3.17-3.16$ (d, 1H), 2.13 (s, 4H), 1.94-1.87 (m, 6H), 0.89$0.80(\mathrm{~m}, 12 \mathrm{H}) ;{ }^{13} \mathrm{C}$ NMR (DMSO-d ${ }_{6}, \delta=39.52 \mathrm{ppm}, 126 \mathrm{MHz}$ ): $\delta 170.35,156.77,150.39,149.67,147.82,137.76,135.00,130.43$, 125.64, 123.38, 121.04, 117.89, 116.19, 113.71, 113.19, 57.99, 54.18, 51.44, 48.61, 46.79, 30.96, 30.87, 29.84, 24.26, 18.51; HRMS (ESI) m/ $z$ : anal. calcd for $[\mathrm{M}+\mathrm{H}]^{+} \mathrm{C}_{40} \mathrm{H}_{51} \mathrm{~N}_{8} \mathrm{O}_{10} \mathrm{~S}$ : 835.3443; found 835.3445.

Methyl ((R)-2-((S)-2-((3-hydroxyphenyl)carbamoyl)pyrrolidin1-yl)-2-oxo-1-phenylethyl)carbamate (25). Yield (839 mg, 79\%); ${ }^{1} \mathrm{H}$ NMR (DMSO-d $\left.{ }_{6}, \delta=2.5 \mathrm{ppm}, 400 \mathrm{MHz}\right): \delta 10.00 / 9.86 / 9.73$ $(3 \mathrm{~s}, 0.20 \mathrm{H}+0.14 \mathrm{H}+0.72 \mathrm{H}), 9.37(\mathrm{~s}, 1 \mathrm{H}), 7.98-7.97 / 7.71-7.69 /$ $7.55-7.52(\mathrm{~d} / \mathrm{d} / \mathrm{m}, 0.34 \mathrm{H}+0.86 \mathrm{H}+0.41 \mathrm{H}), 7.42-7.30(\mathrm{~m}, 4 \mathrm{H})$, 7.20/7.15-7.13 (s/t, 0.70H+0.39H), 7.09-7.05 (t, 1H), 7.00-6.85/ 6.87-6.85 (2 d, 0.73H + 0.19H), 6.47-6.45 (d, 1H), 5.51-5.49/ $5.33-5.31 / 4.92-4.89(3 \mathrm{~d}, 0.74 \mathrm{H}+0.21 \mathrm{H}+0.19 \mathrm{H}), 4.39-4.37(\mathrm{t}$, $1 \mathrm{H}), 3.81(\mathrm{~s}, 1 \mathrm{H}), 3.54(\mathrm{~s}, 3 \mathrm{H}), 3.21-3.15(\mathrm{q}, 1 \mathrm{H}), 2.33-1.77(\mathrm{~m}$, $4 \mathrm{H}) ;{ }^{13} \mathrm{C}$ NMR (DMSO-d 6 , $\delta=39.52 \mathrm{ppm}, 126 \mathrm{MHz}$ ): $\delta 170.09$, 168.34, 157.61, 156.07, 140.03, 137.20, 129.31, 128.61, 128.04, 110.42, 110.04, 106.48, 60.69, 56.71, 51.64, 46.95, 29.34, 24.25; HRMS (ESI) $m / z$ : anal. calcd for $[\mathrm{M}+\mathrm{Na}]^{+} \mathrm{C}_{21} \mathrm{H}_{23} \mathrm{~N}_{3} \mathrm{NaO}_{5}$ : 420.1530; found 420.1531.

Methyl ((S)-1-((S)-2-(5-(3-hydroxyphenyl)-1H-imidazol-2-yl) pyrrolidin-1-yl)-3-methyl-1-oxobutan-2-yl)carbamate (26). Yield (455 mg, 64\%); ${ }^{1} \mathrm{H}$ NMR (DMSO-d ${ }_{6}, \delta=2.5 \mathrm{ppm}, 500 \mathrm{MHz}$ ): $\delta 11.67$ (app br s, 1H), 9.44 (app br s, 1H), 7.36-7.25 (m, 2H), 7.17-6.86 (m, 3H), $6.56(\mathrm{~s}, 1 \mathrm{H}), 5.24-5.23 / 5.08-5.07$ (2 d, $0.13 \mathrm{H}+0.81 \mathrm{H}), 4.08-4.05(\mathrm{t}, 1 \mathrm{H}), 3.80-3.76(\mathrm{~d}, 2 \mathrm{H}), 3.54-3.44$ (app s, 3H), 2.27-2.08 (m, 2H), 2.01-1.86 (m, 3H), 0.89-0.84 (q, 
$6 \mathrm{H}) ;{ }^{13} \mathrm{C}$ NMR (DMSO-d $\left.{ }_{6}, \delta=39.52 \mathrm{ppm}, 126 \mathrm{MHz}\right): \delta 170.43$, $157.61,156.85,129.33,115.06,113.06,111.10$, 58.05, 57.54, 55.19, 54.25, 51.47, 46.87, 30.92, 29.88, 24.31, 19.04, 18.53; HRMS (ESI) $m / z$ : anal. calcd for $[\mathrm{M}+\mathrm{H}]^{+} \mathrm{C}_{20} \mathrm{H}_{27} \mathrm{~N}_{4} \mathrm{O}_{4}$ : 387.2027; found 387.2029.

3-((S)-1-((R)-2-((Methoxycarbonyl)amino)-2-phenylacetyl)pyrrolidine-2-carboxamido)phenyl sulfurofluoridate (27). A mixture of methyl $((R)-2-((S)-2-((3-h y d r o x y p h e n y l)$ carbamoyl)pyrrolidin-1-yl)-2-oxo-1-phenylethyl)carbamate $(276 \mathrm{mg}$, $0.695 \mathrm{mmol})$ and $N, N$-diisopropylethylamine $(363 \mu \mathrm{L}, 2.09$ $\mathrm{mmol})$ in $\mathrm{CH}_{2} \mathrm{Cl}_{2}(10 \mathrm{~mL})$ was stirred under sulfuryl fluoride at room temperature for $5 \mathrm{~h}$. The mixture was then poured in $1 \mathrm{~N}$ aq. $\mathrm{HCl}$ solution and extracted with $\mathrm{CH}_{2} \mathrm{Cl}_{2}$. The organic layer dried over magnesium sulfate, filtered, and concentrated in vacuo. Without any purification, the residue was obtained as a white oil; yield (255 mg, 76\%); ${ }^{1} \mathrm{H}$ NMR (DMSO$\left.\mathrm{d}_{6}, \delta=2.5 \mathrm{ppm}, 400 \mathrm{MHz}\right): \delta 10.58 / 10.51 / 10.41 / 10.34(4 \mathrm{~s}$, $0.02 \mathrm{H}+0.23 \mathrm{H}+0.11 \mathrm{H}+0.53 \mathrm{H}), 7.99-7.86(\mathrm{~m}, 1 \mathrm{H}), 7.74-7.66$ $(\mathrm{m}, 1 \mathrm{H}), 7.63-7.59(\mathrm{t}, 1 \mathrm{H}), 7.56-7.52(\mathrm{t}, 1 \mathrm{H}), 7.42-7.41(\mathrm{~d}, 2 \mathrm{H})$, $7.38-7.35(\mathrm{t}, 2 \mathrm{H}), 7.34-7.32(\mathrm{~m}, 1 \mathrm{H}), 7.29-7.24 / 7.06-7.05 /$ $6.72-6.70(\mathrm{~m} / \mathrm{d} / \mathrm{d}, 0.88 \mathrm{H}+0.28 \mathrm{H}+0.06 \mathrm{H}), 5.52-5.47 / 5.35-$ $5.33 / 4.90-4.88(\mathrm{~m} / \mathrm{d} / \mathrm{d}, 0.88 \mathrm{H}+0.08 \mathrm{H}+0.08 \mathrm{H}), 4.50-4.38(\mathrm{~m}$, $1 \mathrm{H}), 3.83-3.62(\mathrm{~m}, 1 \mathrm{H}), 3.54(\mathrm{~s}, 3 \mathrm{H}), 3.20-3.10(\mathrm{~m}, 1 \mathrm{H}), 2.29-$ $1.78(\mathrm{~m}, 4 \mathrm{H}) ;{ }^{13} \mathrm{C}$ NMR (DMSO-d $\left.6, \delta=39.52 \mathrm{ppm}, 100 \mathrm{MHz}\right)$ : $\delta$ 170.95, 168.40, 156.06, 149.63, 140.92, 137.15, 130.98, 128.58, 128.39, 128.02, 119.34, 115.34, 111.06, 60.69, 56.66, 51.59, 46.94, 29.17, 24.28; ${ }^{19} \mathrm{~F}$ NMR (DMSO-d $\left.6,376 \mathrm{MHz}\right)$ : 38.64; HRMS (ESI) $m / z$ : anal. calcd for $[\mathrm{M}+\mathrm{Na}]^{+} \mathrm{C}_{21} \mathrm{H}_{22} \mathrm{FN}_{3}{ }^{-}$ $\mathrm{NaO}_{7} \mathrm{~S}: 502.1055$; found 502.1054.

Methyl ((S)-1-((S)-2-(5-(3-((tert-butyldimethylsilyl)oxy)-phenyl)-1H-imidazol-2-yl)pyrrolidin-1-yl)-3-methyl-1-oxobutan-2-yl) carbamate (28). A mixture of methyl $((S)-1-((S)-2-(5-(3-h y d r o x-$ yphenyl)-1H-imidazol-2-yl)pyrrolidin-1-yl)-3-methyl-1-oxobutan2-yl)carbamate (158 mg, $0.409 \mathrm{mmol})$, tert-butyldimethylsilyl chloride (123 mg, $0.817 \mathrm{mmol}$ ), imidazole (139 mg, $2.04 \mathrm{mmol}$ ) in $\mathrm{CH}_{2} \mathrm{Cl}_{2}(10 \mathrm{~mL})$ was stirred at room temperature for $4 \mathrm{~h}$. The mixture was then poured in $\mathrm{H}_{2} \mathrm{O}$ and extracted with $\mathrm{CH}_{2} \mathrm{Cl}_{2}$. The organic layer dried over magnesium sulfate, and then was purified by the column chromatography (n-hexane/ethyl acetate). The residue was obtained as sticky white oil; yield (187 mg, 91\%); ${ }^{1} \mathrm{H}$ NMR (DMSO-d ${ }_{6}, \delta=2.5$ ppm, $400 \mathrm{MHz}$ ): $\delta 12.12 / 12.03 / 11.77(3 \mathrm{~s}, 0.16 \mathrm{H}+0.09 \mathrm{H}+0.68 \mathrm{H}), 7.44-7.06(\mathrm{~m}$, $5 \mathrm{H}), 6.69-6.60(\mathrm{~m}, 1 \mathrm{H}), 5.06-5.05(\mathrm{~d}, 1 \mathrm{H}), 4.08-4.04(\mathrm{t}, 1 \mathrm{H}), 3.79$ (s, 2H), $3.53(\mathrm{~s}, 3 \mathrm{H}), 2.16-2.07(\mathrm{~m}, 2 \mathrm{H}), 1.96-1.89(\mathrm{~m}, 3 \mathrm{H}), 0.96$ (s, 9H), 0.92-0.90 (d, 2H), 0.88-0.82 (q, 4H), $0.18(\mathrm{~s}, 6 \mathrm{H}) ;{ }^{13} \mathrm{C}$ NMR (DMSO-d 6 , $\delta=39.52 \mathrm{ppm}, 126 \mathrm{MHz}): \delta 170.30,156.78$, 155.24, 149.15, 138.83, 136.71, 129.36, 117.49, 117.08, 115.60, 112.41, 57.96, 54.21, 51.42, 46.80, 31.01, 29.84, 25.61, 24.13, 19.01, 18.56, 17.96; HRMS (ESI) $m / z$ : anal. calcd For $[\mathrm{M}+\mathrm{H}]^{+}$ $\mathrm{C}_{26} \mathrm{H}_{41} \mathrm{~N}_{4} \mathrm{O}_{4} \mathrm{Si}$ : 501.2892; found 501.2894.

3-(2-((S)-1-((Methoxycarbonyl)-L-valyl)pyrrolidin-2-yl)-1H-imidazol-5-yl)phenyl (3- $((S)-1-((R)-2-(($ methoxycarbonyl)-amino)-2phenylacetyl)pyrrolidine-2-carboxamido)phenyl) sulfate (29). A mixture of $3-((S)-1-((R)-2-(($ methoxycarbonyl $)-a m i n o)-2-$ phenylacetyl)pyrrolidine-2-carboxamido)phenyl sulfurofluoridate (88.9 mg, $0.178 \mathrm{mmol})$, methyl $((S)-1-((S)-2-(5-(3-(($ tert-butyldimethylsilyl)oxy)phenyl)-1 $H$-imidazol-2-yl)pyrrolidin-1-yl)-3-meth- yl-1-oxobutan-2-yl)carbamate (102 $\mathrm{mg}, 0.213 \mathrm{mmol}$ ) and DBU $(34.5 \mu \mathrm{L}, 0.230 \mathrm{mmol})$ in DMF $(10 \mathrm{~mL})$ was stirred at $50{ }^{\circ} \mathrm{C}$ for $12 \mathrm{~h}$. The mixture was then poured in water and extracted with $\mathrm{CH}_{2} \mathrm{Cl}_{2}$. The organic layer dried over magnesium sulfate, and then crude product was purified by the column chromatography ( $n$-hexane/ethyl acetate). The residue was obtained as white solid; yield (97.1 mg, 65\%); ${ }^{1} \mathrm{H}$ NMR (DMSO-d ${ }_{6}, \delta=2.5 \mathrm{ppm}, 500 \mathrm{MHz}$ ): $\delta 11.95(\mathrm{~s}, 1 \mathrm{H}), 10.46 / 10.28(2 \mathrm{~s}, 0.56 \mathrm{H}+0.31 \mathrm{H}), 7.92-7.88(\mathrm{~d}, 1 \mathrm{H})$, 7.82-7.72 (m, 2H), 7.66-7.63 (m, 1H), 7.61-7.58 (t, 1H), 7.49-7.46 $(\mathrm{t}, 1 \mathrm{H}), 7.41-7.40(\mathrm{~d}, 2 \mathrm{H}), 7.38-7.35(\mathrm{t}, 1 \mathrm{H}), 7.33-7.25(\mathrm{~m}, 3 \mathrm{H})$, $7.22-7.20$ (d, 1H), 7.12-7.08 (t, 1H), 7.03-6.63 (m, 1H), 5.51-5.46/ $5.32-5.31 / 5.22-5.20(\mathrm{~m} / \mathrm{d} / \mathrm{d}, 0.76 \mathrm{H}+0.06 \mathrm{H}+0.11 \mathrm{H}), 5.07-5.04 /$ $4.90-4.88(\mathrm{~m} / \mathrm{d}, 0.80 \mathrm{H}+0.05 \mathrm{H}), 4.49-4.37(\mathrm{~m}, 1 \mathrm{H}), 4.06-4.03(\mathrm{t}$, $1 \mathrm{H}), 3.79-3.65$ (m, 2H), 3.53 (s, 6H), 3.45-3.39 (m, 1H), 3.23-3.08 (m, 1H), 2.19-2.08 (m, 2H), 1.95-1.94 (d, 2H), 1.92-1.85 (m, 2H), 1.65-1.55 (m, 3H), 1.23 (s, 0.81H) 0.87-0.81 (m, 6H); ${ }^{13} \mathrm{C}$ NMR (DMSO-d ${ }_{6}, \delta=39.52$ ppm, $126 \mathrm{MHz}$ ): $\delta$ 170.76, 170.34, 168.34, $168.15,156.77,156.29$, 150.38, 149.89, 140.83, 136.78, 130.62, 130.49 , 128.56, 128.33, 128.00, 127.75, 123.42, 118.12, 116.18, 115.30, 111.13, 60.59, 57.96, 56.60, 54.15, 51.41, 46.79, 30.96, 29.83, 29.20, 24.69, 24.19, 18.83, 18.48; HRMS (ESI) $m / z$ : anal. calcd for $[\mathrm{M}+\mathrm{H}]^{+} \mathrm{C}_{41} \mathrm{H}_{48} \mathrm{~N}_{7} \mathrm{O}_{11} \mathrm{~S}$ : 846.3127; found 846.3129.

Methyl ((R)-2-((S)-2-((3-((tert-butyldimethylsilyl)oxy)-phe-nyl) carbamoyl)pyrrolidin-1-yl)-2-oxo-1-phenylethyl)-carbamate (30). A mixture of methyl $((R)-2-((S)-2-((3-h y d r o x y p h e n y l)$ carbamoyl $)$ pyrrolidin-1-yl)-2-oxo-1-phenyl-ethyl)carbamate (51.1 mg, 0.129 mmol), tert-butyldimethylsilyl chloride (29.1 $\mathrm{mg}, 0.193 \mathrm{mmol}$ ), imidazole (26.3 mg, $0.0263 \mathrm{mmol})$ in $\mathrm{CH}_{2} \mathrm{Cl}_{2}(10 \mathrm{~mL})$ was stirred at room temperature for $4 \mathrm{~h}$. The mixture was then poured in $\mathrm{H}_{2} \mathrm{O}$ and extracted with $\mathrm{CH}_{2} \mathrm{Cl}_{2}$. The organic layer dried over magnesium sulfate, and then was purified by the column chromatography ( $n$-hexane/ethyl acetate). The residue was obtained as sticky white oil; yield (53.5 mg, 81\%); ${ }^{1} \mathrm{H}$ NMR (DMSO-d 6 , $\delta=$ $2.5 \mathrm{ppm}, 500 \mathrm{MHz}): \delta 10.08 / 9.82(2 \mathrm{~s}, 0.18 \mathrm{H}+0.81 \mathrm{H}), 7.97-7.96 /$ $7.71-7.69(2 \mathrm{~d}, 0.13 \mathrm{H}+0.81 \mathrm{H}), 7.41-7.31(\mathrm{~m}, 4 \mathrm{H}), 7.26(\mathrm{~s}, 1 \mathrm{H})$, 7.17-6.97 (m, 2H), $6.54(\mathrm{~s}, 1 \mathrm{H}), 5.50-5.48 / 5.32-5.30 / 4.90-4.88$ (3 $\mathrm{d}, 0.84 \mathrm{H}+0.14 \mathrm{H}+0.13 \mathrm{H}), 4.37(\mathrm{~s}, 1 \mathrm{H}), 3.81(\mathrm{~s}, 1 \mathrm{H}), 3.53(\mathrm{~s}, 3 \mathrm{H})$, 3.18-3.16 (d, 1H), 1.98-1.77 (m, 4H), 0.96-0.95 (app s, 9H), 0.200.19 (app s, 6H); ${ }^{13} \mathrm{C}$ NMR (DMSO-d $6, \delta=39.52$ ppm, $126 \mathrm{MHz}$ ): $\delta 170.21,168.29,156.01,155.28,140.18,137.15,129.46,128.56$, 128.00, 127.70, 114.58, 112.27, 110.74, 60.67, 56.66, 51.58, 46.90, 29.26, 25.54, 24.22, 17.89, -4.50; HRMS (ESI) $\mathrm{m} / \mathrm{z}$ : anal. calcd for $[\mathrm{M}+\mathrm{Na}]^{+} \mathrm{C}_{27} \mathrm{H}_{37} \mathrm{~N}_{3} \mathrm{NaO}_{5} \mathrm{Si}$ : 534.2395; found 534.2396.

tert-Butyl (S)-2-((3-)((3- $((S)-1-((R)-2-((m e t h o x y c a r b o n y l)-a m i n o)-2-$ phenylacetyl)pyrrolidine-2-carboxamido)phenoxy)-sulfonyl)oxy)phenyl)carbamoyl)pyrrolidine-1-carboxylate (31). A mixture of methyl $((R)-2-((S)-2-((3-(($ tert-butyldimethylsilyl)-oxy)phenyl)carbamoyl)pyrrolidin-1-yl)-2-oxo-1-phenylethyl)-carbamate $(602 \mathrm{mg}, 1.18$ mmol), tert-butyl (S)-2-((3-((fluorosulfonyl)oxy)phenyl)carbamoyl) pyrrolidine-1-carboxylate $(914 \mathrm{mg}, 0.235 \mathrm{mmol})$ and DBU (35.2 $\mu \mathrm{L}, 0.235 \mathrm{mmol})$ in $\mathrm{DMF}(10 \mathrm{~mL})$ was stirred at $50^{\circ} \mathrm{C}$ for $12 \mathrm{~h}$. The mixture was then poured in $1 \mathrm{~N}$ aq. $\mathrm{HCl}$ solution and extracted with $\mathrm{CH}_{2} \mathrm{Cl}_{2}$. The organic layer dried over magnesium sulfate, and then was purified by the column chromatography ( $n$-hexane/ ethyl acetate). The residue was obtained as white solid; yield (676 mg, 75\%); ${ }^{1} \mathrm{H}$ NMR (DMSO-d $6, \delta=2.5 \mathrm{ppm}, 500 \mathrm{MHz}$ ): $\delta$ 10.44-10.25 (m, 2H), 7.94-7.90 (t, 2H), 7.73-7.71 (d, 1H), 7.66- 
7.57 (m, 2H), 7.49-7.45 (m, 2H), 7.42-7.40 (d, 2H), 7.38-7.24 (m, $3 \mathrm{H}), 7.14-7.04(\mathrm{~m}, 2 \mathrm{H}), 5.52-5.47 / 5.35-5.33 / 4.91-4.89(\mathrm{~m} / \mathrm{d} / \mathrm{d}$, $0.83 \mathrm{H}+0.10 \mathrm{H}+0.09 \mathrm{H}), 4.51-4.38(\mathrm{~m}, 1 \mathrm{H}), 4.27-4.25 / 4.20-4.17$ $(\mathrm{d} / \mathrm{q}, 0.35 \mathrm{H}+0.64 \mathrm{H}), 4.14-4.10(\mathrm{q}, 1 \mathrm{H}), 3.82(\mathrm{~s}, 1 \mathrm{H}), 3.53(\mathrm{~s}, 3 \mathrm{H})$, 3.46-3.42 (m, 1H), 3.37-3.34 (m, 1H) 3.19-3.17 (d, 3H), 2.24-2.13 $(\mathrm{m}, 1 \mathrm{H}), 2.05-1.78(\mathrm{~m}, 7 \mathrm{H}), 1.39-1.24$ (app s, 9H); ${ }^{13} \mathrm{C}$ NMR (DMSO-d ${ }_{6}, \delta=39.52 \mathrm{ppm}, 126 \mathrm{MHz}$ ): $\delta 172.03,170.78,168.35$, $153.06,149.85,140.74,130.66,128.57,128.34,128.00,118.40$, $115.34,111.40,78.60,60.46,56.64,51.61,46.56,30.91,29.19$, 27.89, 24.27, 23.39; HRMS (ESI) $m / z$ : anal. calcd for $[\mathrm{M}+\mathrm{Na}]^{+}$ $\mathrm{C}_{37} \mathrm{H}_{43} \mathrm{~N}_{5} \mathrm{NaO}_{11} \mathrm{~S}$ : 788.2572; found 788.2575.

3-((S)-1-((R)-2-((Methoxycarbonyl)amino)-2-phenylacetyl)-pyrrolidine-2-carboxamido)phenyl (3- $((S)-1-(5-((3 a S, 4 S, 6 a R)-2-o x o h e x-$ ahydro-1H-thieno[3,4- $d$ ]imidazol-4-yl)pentanoyl)-pyrrolidine-2-carboxamido)phenyl) sulfate (32). A mixture of tert-butyl $(S)-2-((3-$ $(((3-((S)-1-((R)-2-(($ methoxycarbonyl)amino)-2-phenylacetyl) pyrrolidine-2-carboxamido)phenoxy)sulfonyl)-oxy)phenyl) carbamoyl)pyrrolidine-1-carboxylate (426 $\mathrm{mg}, 0.557 \mathrm{mmol}$ ) in $\mathrm{CF}_{3} \mathrm{CO}_{2} \mathrm{H}(5 \mathrm{~mL}) / \mathrm{CH}_{2} \mathrm{Cl}_{2}(5 \mathrm{~mL})$ was stirred at room temperature for $30 \mathrm{~m}$. The volatile component was removed in vacuo, 1-ethyl-3-(3-dimethylaminopropyl)-carbodiimide (139 mg, $0.724 \mathrm{mmol}$ ), hydroxybenzotriazole hydrate (97.8 mg, $0.724 \mathrm{mmol}), 5-[(3 a S, 4 S, 6 a R)$-2-oxohexahydro- $1 H$ thieno[3,4- $d$ ]imidazol-4-yl]pentanoic acid (163 mg, 0.668 $\mathrm{mmol}$ ) were added in batches over $4 \mathrm{~min}$ to a solution of $N, N$-diisopropylethylamine $(485 \mu \mathrm{L}, 2.78 \mathrm{mmol})$ in $\mathrm{CH}_{2} \mathrm{Cl}_{2}$ $(10 \mathrm{~mL})$ and the reaction mixture stirred at room temperature for overnight. The mixture was then poured in $1 \mathrm{~N}$ aq. $\mathrm{HCl}$ solution and extracted with $\mathrm{CH}_{2} \mathrm{Cl}_{2}$. The organic layer dried over magnesium sulfate, and then was purified by the column chromatography ( $n$-hexane/EA). The residue was obtained as white solid; yield (479 mg, 96\%); ${ }^{1} \mathrm{H}$ NMR (DMSO-d ${ }_{6}, \delta=2.5$ ppm, $500 \mathrm{MHz}$ ): $\delta$ 10.52/10.45/10.36/10.27 $(4 \mathrm{~s}, 0.38 \mathrm{H}+0.53 \mathrm{H}+0.89 \mathrm{H}+0.31 \mathrm{H}), 7.91-7.84(\mathrm{~m}, 2 \mathrm{H})$, $7.75-7.58(\mathrm{~m}, 3 \mathrm{H}), 7.50-7.44(\mathrm{~m}, 2 \mathrm{H}), 7.41-7.29(\mathrm{~m}, 5 \mathrm{H})$, 7.23-7.04 (m, 2H), $6.44(\mathrm{~s}, 1 \mathrm{H}), 6.39-6.34(\mathrm{t}, 1 \mathrm{H}), 5.50-5.45 /$ $5.32-5.30 / 5.21-5.19 / 5.09 / 4.88-4.87(\mathrm{q} / \mathrm{d} / \mathrm{d} / \mathrm{s} / \mathrm{d}, \quad 0.84 \mathrm{H}+$ $0.07 \mathrm{H}+0.05 \mathrm{H}+0.03 \mathrm{H}+0.10 \mathrm{H}), 4.54-4.25(\mathrm{~m}, 4 \mathrm{H}), 4.13-$ $4.07(\mathrm{~m}, 1 \mathrm{H}), 3.81-3.56(\mathrm{~m}, 3 \mathrm{H}), 3.53(\mathrm{~s}, 3 \mathrm{H}), 3.20-3.00(\mathrm{~m}$, $2 \mathrm{H}), 2.82-2.74(\mathrm{~m}, 1 \mathrm{H}), 2.58-2.56(\mathrm{~d}, 1 \mathrm{H}), 2.33-2.26(\mathrm{~m}, 2 \mathrm{H})$, 2.17-2.12 (m, 1H), 2.17-1.96 (m, 2H), 1.91-1.81 (m, 5H), $1.64-1.58(\mathrm{~m}, 1 \mathrm{H}), 1.51-1.44(\mathrm{~m}, 3 \mathrm{H}), 1.40-1.33(\mathrm{~m}, 2 \mathrm{H})$, $1.23(\mathrm{~s}, 1 \mathrm{H}) ;{ }^{13} \mathrm{C}$ NMR (DMSO-d ${ }_{6}, \delta=39.52 \mathrm{ppm}, 126 \mathrm{MHz}$ ): $\delta 171.41,170.81,168.35,162.71,156.31,149.87,140.88$, $136.79,130.62,128.57,128.33,128.00,118.22,115.23$, $113.71,111.17,61.05,60.61,60.01,59.19,56.61,55.47$, 51.53, 46.93, 33.35, 29.47, 29.20, 28.20, 24.46, 24.24; HRMS (ESI) $m / z$ : anal. calcd for $[\mathrm{M}+\mathrm{Na}]^{+} \mathrm{C}_{42} \mathrm{H}_{49} \mathrm{~N}_{7} \mathrm{NaO}_{11} \mathrm{~S}_{2}$ : 915.0012; found 914.2826 .

\section{Measurement of the anti-HCV activities of compounds using HCVec}

Cell line and cell culture. Huh 7.5.1 cells were grown in Dulbecco's Modified Eagle's medium (DMEM; Gibco) containing antibiotics (100 $\mathrm{U} \mathrm{mL}^{-1}$ penicillin, $10 \mathrm{mg} \mathrm{mL}^{-1}$ streptomycin) and $10 \%$ heat-inactivated fetal bovine serum (DFBS; Hyclone) at $37{ }^{\circ} \mathrm{C}$ in a humidified $6.0 \% \mathrm{CO}_{2}$ incubator.

\section{Virus production}

In vitro transcribed RNA of JFH5a-Rluc-ad34, which is a derivative of JFH1 with adaptive mutations in the E2 and p7 regions, was transfected into Huh7.5.1 cells by electroporation. JFH5aRluc-ad34 virus contains a reporter Renilla luciferase (Rluc) for convenient virus proliferation assay. ${ }^{92} \mathrm{HCV}$-containing cell culture media were collected 3-5 days after electroporation and filtered through a $0.45 \mathrm{mM}$ pore size filter.

\section{Antiviral activity test with infectious HCV particles}

Huh 7.5.1 cells were inoculated with a JFH5a-Rluc-ad34 virus stock and cultivated for $3 \mathrm{~h}$. At $3 \mathrm{~h}$ after the virus inoculation, HCV infected Huh7.5.1 cells were cultured with media containing serially diluted compounds. At 3 days after the chemical treatment, the cells were harvested and their luciferase activities were measured using a Renilla luciferase assay system (Promega) according to the manufacturer's direction. Finally, the luciferase activities were normalized to those obtained from mock-treated cells.

\section{Cell line and cell culture}

Huh 7.5.1 cells containing a bicistronic HCV replicon NK5.1Gluc cell line (genotype $1 \mathrm{~b}$ ) were employed for the interrogation of the anti-HCV activities of the compounds. ${ }^{64}$ The first open reading frame (ORF) of the replicon contains the Gaussia luciferase gene fused with foot-and-mouth disease virus (FMDV) 2a gene and the neomycin phosphotransferase gene, and the second ORF contains HCV nonstructural genes NS3-5. The replicon-containing cells were cultivated under the same conditions as described above in presence of an additional antibiotic G418 (0.5 $\mathrm{mg} \mathrm{mL}^{-1}$, Calbiochem).

\section{Antiviral activity assay test with HCV replicon}

Huh7.5.1 cells containing HCV replicon (NK5.1-Gluc) were planted on a 12-well plate. At $16 \mathrm{~h}$ after cultivation, the replicon containing NK/R2AN-containing cells were incubated with media containing serially diluted compounds for 3 days. After the chemical treatment, the cell culture media were collected and luciferase activities were measured through the use of the Renilla luciferase assay system (Promega) according to manufacturer's direction, and then normalized to those obtained from mock-treated cells.

\section{Molecular docking}

The X-ray structure of 3FQQ and NMR structure of $1 \mathrm{R} 7 \mathrm{G}$ were retrieved from the protein data bank (PDB) and prepared via homology modeling with Discovery Studio Client. The default ligand settings were used to prepare all tautomers, and further energy minimized. The inhibitors were then docked into each of the NS5A dimer models using the CDOCKER. 


\section{Conflicts of interest}

There are no conflicts of interest to declare regarding this study.

\section{Acknowledgements}

We gratefully acknowledge the financial support of the Bio R\&D Program (No. 2012M3A9A9054974 and No. 2017M3A9F6029755) through the National Research Foundation funded by the MSIT, Republic of Korea. This research also was supported by the Korea Institute of Science and Technology Information (KISTI) Institutional Program.

\section{Notes and references}

1 A. M. Di Bisceglie, Lancet, 1998, 351, 351-355.

2 A. M. Di Bisceglie, Hepatology, 2000, 31, 1014-1018.

3 W. R. Kim, Microbes Infect., 2002, 4, 1219-1225.

4 N. Horscroft, V. C. H. Lai, W. Cheney, N. Yao, J. Z. Wu, Z. Hong and W. Zhong, Antiviral Chem. Chemother., 2005, 16, 1-12.

5 S. A. Qureshi, Med. Res. Rev., 2007, 27, 353-373.

6 T. O. Moore, M. Paradowski and S. E. Ward, Org. Biomol. Chem., 2016, 14, 3307-3313.

7 L. Tong, W. Yu, L. Chen, O. Selyutin, M. P. Dwyer, A. G. Nair, R. Mazzola, J. H. Kim, D. Sha, J. Yin, R. T. Ruck, I. W. Davies, B. Hu, B. Zhong, J. Hao, T. Ji, S. Zan, R. Liu, S. Agrawal, E. Xia, S. Curry, P. McMonagle, K. Bystol, F. Lahser, D. Carr, L. Rokosz, P. Ingravallo, S. Chen, K. I. Feng, M. Cartwright, E. Asante-Appiah and J. A. Kozlowski, J. Med. Chem., 2017, 60, 290-306.

8 K. J. Blight, A. A. Kolykhalov and C. M. Rice, Science, 2000, 290, 1972-1974.

9 C. Liang, E. Rieder, B. Hahm, S. K. Jang, A. Paul and E. Wimmer, Virology, 2005, 333, 41-53.

10 T. W. Bell, ChemMedChem, 2010, 5, 1663-1665.

11 W. Cun, J. Jiang and G. Luo, J. Virol., 2010, 84, 11532-11541. 12 J. L. Romine, D. R. St. Laurent, J. E. Leet, S. W. Martin, M. H. Serrano-Wu, F. Yang, M. Gao, D. R. O'Boyle, J. A. Lemm, J.-H. Sun, P. T. Nower, X. Huang, M. S. Deshpande, N. A. Meanwell and L. B. Snyder, ACS Med. Chem. Lett., 2011, 2, 224-229.

13 D. A. DeGoey, J. T. Randolph, D. Liu, J. Pratt, C. Hutchins, P. Donner, A. C. Krueger, M. Matulenko, S. Patel, C. E. Motter, L. Nelson, R. Keddy, M. Tufano, D. D. Caspi, P. Krishnan, N. Mistry, G. Koev, T. J. Reisch, R. Mondal, T. Pilot-Matias, Y. Gao, D. W. Beno, C. J. Maring, A. Molla, E. Dumas, A. Campbell, L. Williams, C. Collins, R. Wagner and W. M. Kati, J. Med. Chem., 2014, 57, 20472057.

14 M. S. Sulkowski, D. F. Gardiner, M. Rodriguez-Torres, K. R. Reddy, T. Hassanein, I. Jacobson, E. Lawitz, A. S. Lok, F. Hinestrosa, P. J. Thuluvath, H. Schwartz, D. R. Nelson, G. T. Everson, T. Eley, M. Wind-Rotolo, S.-P. Huang, M. Gao, D. Hernandez, F. McPhee, D. Sherman, R. Hindes, W. Symonds, C. Pasquinelli and D. M. Grasela, N. Engl. J. Med., 2014, 370, 211-221.
15 T. I. Ng, P. Krishnan, T. Pilot-Matias, W. Kati, G. Schnell, J. Beyer, T. Reisch, L. Lu, T. Dekhtyar, M. Irvin, R. Tripathi, C. Maring, J. T. Randolph, R. Wagner and C. Collins, Antimicrob. Agents Chemother., 2017, 61, e02558-16.

16 B. Liu, K. Gai, H. Qin, X. Liu, Y. Cao, Q. Lu, D. Lu, D. Chen, H. Shen, W. Song, Y. Zhang, X. Wang, H. Xu and Y. Zhang, Eur. J. Med. Chem., 2018, 148, 95-105.

17 S. L. Tan, A. Pause, Y. Shi and N. Sonenberg, Nat. Rev. Drug Discovery, 2002, 1, 867-881.

18 R. De Francesco and G. Migliaccio, Nature, 2005, 436, 953960.

19 K. Garber, Nat. Biotechnol., 2011, 29, 963-966.

20 P. J. Pockros, Drugs, 2012, 72, 1825-1831.

21 J. J. Kiser and C. Flexner, Annu. Rev. Pharmacol. Toxicol., 2013, 53, 427-449.

22 M. Zhong, E. Peng, N. Huang, Q. Huang, A. Huq, M. Lau, R. Colonno and L. Li, Bioorg. Med. Chem. Lett., 2014, 24, 5738-5742.

23 S. L. Tan and M. G. Katze, Virology, 2001, 284, 1-12.

24 T. L. Tellinghuisen, M. J. Evans, T. von Hahn, S. You and C. M. Rice, J. Virol., 2007, 81, 8853-8867.

25 R. Bartenschlager, F. Penin, V. Lohmann and P. Andre, Trends Microbiol., 2011, 19, 95-103.

26 J. Schlutter, Nature, 2011, 474, S5-S7.

27 M. Belema, V. N. Nguyen, D. R. St. Laurent, O. D. Lopez, Y. Qiu, A. C. Good, P. T. Nower, L. Valera, D. R. O'Boyle, J.-H. Sun, M. Liu, R. A. Fridell, J. A. Lemm, M. Gao, J. O. Knipe, N. A. Meanwell and L. B. Snyder, Bioorg. Med. Chem. Lett., 2013, 23, 4428-4435.

28 M. Issur and M. Götte, Viruses, 2014, 6, 4227-4241.

29 J. H. Nettles, R. A. Stanton, J. Broyde, F. Amblard, H. Zhang, L. Zhou, J. Shi, T. R. McBrayer, T. Whitaker, S. J. Coats, J. J. Kohler and R. F. Schinazi, J. Med. Chem., 2014, 57, 10031-10043.

30 D. Lavanchy, Liver Int., 2009, 29(suppl. 1), 74-81.

31 E. R. Feeney and R. T. Chung, BMJ, 2014, 348, g3308.

32 R. Wagner, J. T. Randolph, S. V. Patel, L. Nelson, M. A. Matulenko, R. Keddy, J. K. Pratt, D. Liu, A. C. Krueger, P. L. Donner, D. K. Hutchinson, C. Flentge, D. Betebenner, T. Rockway, C. J. Maring, T. I. Ng, P. Krishnan, T. Pilot-Matias, C. Collins, N. Panchal, T. Reisch, T. Dekhtyar, R. Mondal, D. F. Stolarik, Y. Gao, W. Gao, D. A. Beno and W. M. Kati, J. Med. Chem., 2018, 61, 4052-4066.

33 N. A. Terrault, S. Zeuzem, A. M. Di Bisceglie, J. K. Lim, P. J. Pockros, L. M. Frazier, A. Kuo, A. S. Lok, M. L. Shiffman, Z. Ben Ari, L. Akushevich, M. Vainorius, M. S. Sulkowski, M. W. Fried, D. R. Nelson and H.-T. S. Group, Gastroenterology, 2016, 151, 1131-1140.

34 A. Geddawy, Y. F. Ibrahim, N. M. Elbahie and M. A. Ibrahim, J. Transl. Int. Med., 2017, 5, 8-17.

35 D. J. Cada, J. Leonard, T. L. Levien and D. E. Baker, Hosp. Pharm., 2015, 50, 396-412.

36 A. Tamori, M. Enomoto and N. Kawada, Mediators Inflammation, 2016, 2016, 6841628. 
37 J. R. King and R. M. Menon, Clin. Pharmacol. Drug Dev., 2017, 6, 201-205.

38 P. McCormack, Drugs, 2015, 75, 515-524.

39 A. M. Bell, J. L. Wagner, K. E. Barber and K. R. Stover, Int. J. Hepatol., 2016, 2016, 3852126.

40 S. M. McConachie, S. M. Wilhelm and P. B. Kale-Pradhan, Expert Rev. Clin. Pharmacol., 2016, 9, 287-302.

$41 \mathrm{~J}$. Yu, Z. Zhou, K. H. Owens, T. K. Ritchie and I. RagueneauMajlessi, Drug Metab. Dispos., 2017, 45, 86-108.

42 E. Mogalian, P. German, B. P. Kearney, C. Y. Yang, D. Brainard, J. Link, J. McNally, L. Han, J. Ling and A. Mathias, Antimicrob. Agents Chemother., 2017, 61, e02084-16.

43 E. B. Chahine, D. Kelley and L. M. Childs-Kean, Ann. Pharmacother., 2018, 52, 352-363.

44 M. Gao, R. E. Nettles, M. Belema, L. B. Snyder, V. N. Nguyen, R. A. Fridell, M. H. Serrano-Wu, D. R. Langley, J. H. Sun, D. R. O'Boyle 2nd, J. A. Lemm, C. Wang, J. O. Knipe, C. Chien, R. J. Colonno, D. M. Grasela, N. A. Meanwell and L. G. Hamann, Nature, 2010, 465, 96-100.

45 R. E. Nettles, M. Gao, M. Bifano, E. Chung, A. Persson, T. C. Marbury, R. Goldwater, M. P. DeMicco, M. Rodriguez-Torres, A. Vutikullird, E. Fuentes, E. Lawitz, J. C. Lopez-Talavera and D. M. Grasela, Hepatology, 2011, 54, 1956-1965.

46 M. Belema, O. D. Lopez, J. A. Bender, J. L. Romine, D. R. St Laurent, D. R. Langley, J. A. Lemm, D. R. O'Boyle 2nd, J. H. Sun, C. Wang, R. A. Fridell and N. A. Meanwell, J. Med. Chem., 2014, 57, 1643-1672.

47 D. R. St Laurent, M. H. Serrano-Wu, M. Belema, M. Ding, H. Fang, M. Gao, J. T. Goodrich, R. G. Krause, J. A. Lemm, M. Liu, O. D. Lopez, V. N. Nguyen, P. T. Nower, D. R. O'Boyle 2nd, B. C. Pearce, J. L. Romine, L. Valera, J. H. Sun, Y. K. Wang, F. Yang, X. Yang, N. A. Meanwell and L. B. Snyder, J. Med. Chem., 2014, 57, 1976-1994.

48 N. A. Meanwell, J. Med. Chem., 2016, 59, 7311-7351.

49 C. Wang, H. Huang, L. Valera, J.-H. Sun, D. R. O'Boyle II, P. T. Nower, L. Jia, D. Qiu, X. Huang, A. Altaf, M. Gao and R. A. Fridell, Antimicrob. Agents Chemother., 2012, 56, 1350-1358.

50 A. Aghemo and R. De Francesco, Hepatology, 2013, 58, 428438.

51 E. De Clercq, Biochem. Pharmacol., 2014, 89, 441-452.

52 S. Pol and M. Corouge, Med. Mal. Infect., 2014, 44, 449-454.

53 A. Hill, B. Simmons, D. Gotham and J. Fortunak, J. Virus Erad., 2016, 2, 28-31.

54 P. J. Pockros, Expert Opin. Biol. Ther., 2011, 11, 1611-1622. 55 C. Sheridan, Nat. Biotechnol., 2011, 29, 553-554.

56 N. M. Dabbouseh and D. M. Jensen, Nat. Rev. Gastroenterol. Hepatol., 2013, 10, 268-276.

57 J. A. Henderson, D. Bilimoria, M. Bubenik, C. Cadilhac, K. M. Cottrell, F. Denis, E. Dietrich, N. Ewing, G. Falardeau, S. Giroux, L. L'Heureux, B. Liu, N. Mani, M. Morris, O. Nicolas, O. Z. Pereira, C. Poisson, T. J. Reddy, S. Selliah, R. S. Shawgo, L. Vaillancourt, J. Wang, J. Xu, N. Chauret, F. Berlioz-Seux, L. C. Chan,
S. K. Das, A. L. Grillot, Y. L. Bennani and J. P. Maxwell, Bioorg. Med. Chem. Lett., 2015, 25, 948-951.

58 I. J. Kang, S. J. Hsu, H. Y. Yang, T. K. Yeh, C. C. Lee, Y. C. Lee, Y. W. Tian, J. S. Song, T. A. Hsu, Y. S. Chao, A. Yueh and J. H. Chern, J. Med. Chem., 2017, 60, 228-247. 59 R. A. Fridell, C. Wang, J. H. Sun, D. R. O'Boyle 2nd, P. Nower, L. Valera, D. Qiu, S. Roberts, X. Huang, B. Kienzle, M. Bifano, R. E. Nettles and M. Gao, Hepatology, 2011, 54, 1924-1935.

60 E. Poveda, D. L. Wyles, Á. Mena, J. D. Pedreira, Á. CastroIglesias and E. Cachay, Antiviral Res., 2014, 108, 181-191.

61 C. Wang, L. Jia, D. R. O'Boyle 2nd, J. H. Sun, K. Rigat, L. Valera, P. Nower, X. Huang, B. Kienzle, S. Roberts, M. Gao and R. A. Fridell, Antimicrob. Agents Chemother., 2014, 58, 5155-5163.

62 D. Ross-Thriepland and M. Harris, J. Gen. Virol., 2015, 96, 727-738.

63 J. H. Sun, D. R. O'Boyle 2nd, R. A. Fridell, D. R. Langley, C. Wang, S. B. Roberts, P. Nower, B. M. Johnson, F. Moulin, M. J. Nophsker, Y. K. Wang, M. Liu, K. Rigat, Y. Tu, P. Hewawasam, J. Kadow, N. A. Meanwell, M. Cockett, J. A. Lemm, M. Kramer, M. Belema and M. Gao, Nature, 2015, 527, 245-248.

64 Y. You, H. S. Kim, I. H. Bae, S. G. Lee, M. H. Jee, G. Keum, S. K. Jang and B. M. Kim, Eur. J. Med. Chem., 2017, 125, 87-100. 65 A. V. Ivachtchenko, O. D. Mitkin, P. M. Yamanushkin, I. V. Kuznetsova, E. A. Bulanova, N. A. Shevkun, A. G. Koryakova, R. N. Karapetian, V. V. Bichko, A. S. Trifelenkov, D. V. Kravchenko, N. V. Vostokova, M. S. Veselov, N. V. Chufarova and Y. A. Ivanenkov, J. Med. Chem., 2014, 57, 7716-7730.

66 W. M. Kazmierski, A. Maynard, M. Duan, S. Baskaran, J. Botyanszki, R. Crosby, S. Dickerson, M. Tallant, R. Grimes, R. Hamatake, M. Leivers, C. D. Roberts and J. Walker, J. Med. Chem., 2014, 57, 2058-2073.

67 J. J. Kohler, J. H. Nettles, F. Amblard, S. J. Hurwitz, L. Bassit, R. A. Stanton, M. Ehteshami and R. F. Schinazi, Infect. Drug Resist., 2014, 7, 41-56.

68 S. Giroux, D. Bilimoria, C. Cadilhac, K. M. Cottrell, F. Denis, E. Dietrich, N. Ewing, J. A. Henderson, L. L'Heureux, N. Mani, M. Morris, O. Nicolas, T. J. Reddy, S. Selliah, R. S. Shawgo, J. Xu, N. Chauret, F. Berlioz-Seux, L. C. Chan, S. K. Das, A.-L. Grillot, Y. L. Bennani and J. P. Maxwell, Bioorg. Med. Chem. Lett., 2015, 25, 940-943.

69 J. O. Link, J. G. Taylor, L. Xu, M. Mitchell, H. Guo, H. Liu, D. Kato, T. Kirschberg, J. Sun, N. Squires, J. Parrish, T. Keller, Z.-Y. Yang, C. Yang, M. Matles, Y. Wang, K. Wang, G. Cheng, Y. Tian, E. Mogalian, E. Mondou, M. Cornpropst, J. Perry and M. C. Desai, J. Med. Chem., 2014, 57, 2033-2046.

70 E. P. Gillis, K. J. Eastman, M. D. Hill, D. J. Donnelly and N. A. Meanwell, J. Med. Chem., 2015, 58, 8315-8359.

71 F. Amblard, H. Zhang, L. Zhou, J. Shi, D. R. Bobeck, J. H. Nettles, S. Chavre, T. R. McBrayer, P. Tharnish, T. Whitaker, S. J. Coats and R. F. Schinazi, Bioorg. Med. Chem. Lett., 2013, 23, 2031-2034. 
72 S. Giroux, J. Xu, T. J. Reddy, M. Morris, K. M. Cottrell, C. Cadilhac, J. A. Henderson, O. Nicolas, D. Bilimoria, F. Denis, N. Mani, N. Ewing, R. Shawgo, L. L'Heureux, S. Selliah, L. Chan, N. Chauret, F. Berlioz-Seux, M. N. Namchuk, A. L. Grillot, Y. L. Bennani, S. K. Das and J. P. Maxwell, ACS Med. Chem. Lett., 2014, 5, 240-243.

73 D. B. Ascher, J. Wielens, T. L. Nero, L. Doughty, C. J. Morton and M. W. Parker, Sci. Rep., 2014, 4, 4765.

74 J. Dong, L. Krasnova, M. G. Finn and K. B. Sharpless, Angew. Chem., Int. Ed. Engl., 2014, 53, 9430-9448.

75 J. Dong, K. B. Sharpless, L. Kwisnek, J. S. Oakdale and V. V. Fokin, Angew. Chem., Int. Ed. Engl., 2014, 53, 9466-9470.

76 S. Li, L. T. Beringer, S. Chen and S. Averick, Polymer, 2015, 78, 37-41.

77 A. Narayanan and L. H. Jones, Chem. Sci., 2015, 6, 26502659.

78 J. Yatvin, K. Brooks and J. Locklin, Chem.-Eur. J., 2016, 22, 16348-16354.

79 J. Yatvin, K. Brooks and J. Locklin, Angew. Chem., Int. Ed. Engl., 2015, 54, 13370-13373.

80 A. Dondoni and A. Marra, Org. Biomol. Chem., 2017, 15, 1549-1553.

81 B. Gao, L. Zhang, Q. Zheng, F. Zhou, L. M. Klivansky, J. Lu, Y. Liu, J. Dong, P. Wu and K. B. Sharpless, Nat. Chem., 2017, 9, 1083-1088.

82 K. S. Lim, D. W. Kang, Y. S. Kim, M. S. Kim, S. G. Park, S. Choi, L. V. Pearce, P. M. Blumberg and J. Lee, Bioorg. Med. Chem. Lett., 2011, 21, 299-302.

83 J. Shi, L. Zhou, F. Amblard, D. R. Bobeck, H. Zhang, P. Liu, L. Bondada, T. R. McBrayer, P. M. Tharnish, T. Whitaker, S. J. Coats and R. F. Schinazi, Bioorg. Med. Chem. Lett., 2012, 22, 3488-3491.

84 C. A. Coburn, P. T. Meinke, W. Chang, C. M. Fandozzi, D. J. Graham, B. Hu, Q. Huang, S. Kargman, J. Kozlowski, R. Liu, J. A. McCauley, A. A. Nomeir, R. M. Soll, J. P. Vacca, D. Wang, H. Wu, B. Zhong, D. B. Olsen and S. W. Ludmerer, ChemMedChem, 2013, 8, 1930-1940.

85 M. Zhong, E. Peng, N. Huang, Q. Huang, A. Huq, M. Lau, R. Colonno and L. Li, Bioorg. Med. Chem. Lett., 2014, 24, 5731-5737.

86 L. C. King and G. K. Ostrum, J. Org. Chem., 1964, 29, 34593461.

87 S. D. Schimler, M. A. Cismesia, P. S. Hanley, R. D. Froese, M. J. Jansma, D. C. Bland and M. S. Sanford, J. Am. Chem. Soc., 2017, 139, 1452-1455.

88 R. Zelli, S. Tommasone, P. Dumy, A. Marra and A. Dondoni, Eur. J. Org. Chem., 2016, 5102-5116.

89 B. Kim, C.-E. Yeom, H. Kim and S. Lee, Synlett, 2007, 1, 0146-0150.

90 I. H. Bae, J. K. Choi, C. Chough, S. J. Keum, H. Kim, S. K. Jang and B. M. Kim, ACS Med. Chem. Lett., 2014, 5, 255-258.

91 I. H. Bae, H. S. Kim, Y. You, C. Chough, W. Choe, M. K. Seon, S. G. Lee, G. Keum, S. K. Jang and B. Moon Kim, Eur. J. Med. Chem., 2015, 101, 163-178.
92 C. S. Kim, S. J. Keum and S. K. Jang, PLoS One, 2011, 6, e22808.

93 P. J. Lim, U. Chatterji, D. Cordek, S. D. Sharma, J. A. GarciaRivera, C. E. Cameron, K. Lin, P. Targett-Adams and P. A. Gallay, J. Biol. Chem., 2012, 287, 30861-30873.

94 B. D. Lindenbach, M. J. Evans, A. J. Syder, B. Woelk, T. L. Tellinghuisen, C. C. Liu, T. Maruyama, R. O. Hynes, D. R. Burton, J. A. McKeating and C. M. Rice, Science, 2005, 309, 623-626.

95 G. Randall, M. Panis, J. D. Cooper, T. L. Tellinghuisen, K. E. Sukhodolets, S. Pfeffer, M. Landthaler, P. Landgrafe, S. Kan, B. D. Lindenbach, M. Chien, D. B. Weir, J. J. Russo, J. Ju, M. J. Brownstein, R. Sheridan, C. Sander, M. Zavolan, T. Tuschl and C. M. Rice, Proc. Natl. Acad. Sci. U. S. A., 2007, 104, 12884-12889.

96 O. D. Lopez, V. N. Nguyen, D. R. St. Laurent, M. Belema, M. H. Serrano-Wu, J. T. Goodrich, F. Yang, Y. Qiu, A. S. Ripka, P. T. Nower, L. Valera, M. Liu, D. R. O'Boyle, J.-H. Sun, R. A. Fridell, J. A. Lemm, M. Gao, A. C. Good, N. A. Meanwell and L. B. Snyder, Bioorg. Med. Chem. Lett., 2013, 23, 779-784.

97 M. Belema, V. N. Nguyen, J. L. Romine, D. R. St. Laurent, O. D. Lopez, J. T. Goodrich, P. T. Nower, D. R. O'Boyle II, J. A. Lemm, R. A. Fridell, M. Gao, H. Fang, R. G. Krause, Y.-K. Wang, A. J. Oliver, A. C. Good, J. O. Knipe, N. A. Meanwell and L. B. Snyder, J. Med. Chem., 2014, 57, 1995-2012.

98 S. Giroux, D. Bilimoria, C. Cadilhac, K. M. Cottrell, F. Denis, E. Dietrich, N. Ewing, J. A. Henderson, L. L'Heureux, N. Mani, M. Morris, O. Nicolas, T. J. Reddy, S. Selliah, R. S. Shawgo, J. Xu, N. Chauret, F. Berlioz-Seux, L. C. Chan, S. K. Das, A. L. Grillot, Y. L. Bennani and J. P. Maxwell, Bioorg. Med. Chem. Lett., 2015, 25, 936-939.

99 S. Li, D. Cohen-Karni, L. T. Beringer, C. Wu, E. Kallick, H. Edington, M. J. Passineau and S. Averick, Polymer, 2016, 99, 7-12.

100 P. Targett-Adams, E. J. S. Graham, J. Middleton, A. Palmer, S. M. Shaw, H. Lavender, P. Brain, T. D. Tran, L. H. Jones, F. Wakenhut, B. Stammen, D. Pryde, C. Pickford and M. Westby, J. Virol., 2011, 85, 6353-6368.

101 M. Belema and N. A. Meanwell, J. Med. Chem., 2014, 57, 5057-5071.

102 D. R. O'Boyle Ii, J. H. Sun, P. T. Nower, J. A. Lemm, R. A. Fridell, C. Wang, J. L. Romine, M. Belema, V. N. Nguyen, D. R. Laurent, M. Serrano-Wu, L. B. Snyder, N. A. Meanwell, D. R. Langley and M. Gao, Virology, 2013, 444, 343-354.

103 R. A. Love, O. Brodsky, M. J. Hickey, P. A. Wells and C. N. Cronin, J. Virol., 2009, 83, 4395-4403.

104 F. Penin, V. Brass, N. Appel, S. Ramboarina, R. Montserret, D. Ficheux, H. E. Blum, R. Bartenschlager and D. Moradpour, J. Biol. Chem., 2004, 279, 40835-40843. 